\title{
Radiation Transport
}

April 1, 1983—December 31, 1983

R. D. O'Dell 


\section{DISCLAIMER}

This report was prepared as an account of woik sponsored by an agency of the United States Government. Neither the United States Government nor any agency thereof, nor any of their employees, makes any warranty, express or implied, or assumes any legal liability or responsibility for the accuracy, completeness, or usefulness of any information, apparalus, product, or process disclosed, or zepresents that its use woxld not infringe privately owned rights. Reference herein to any specific commercial product. process, or service by trade name, trademark, manufacturer, or otherwise does not necessarily constitute or imply its endorsement, recommendation, or favoring by the United States Government or any agency thereof. The views and opinions of authors expressed herein do not necessarily state or reflect those of the United States Government or any agency thereof.

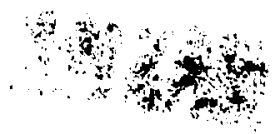


CONTENTS

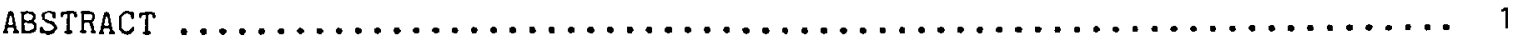

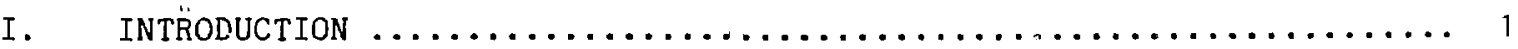

II. FISSION REACTOR NEUTRONICS $\ldots \ldots \ldots \ldots \ldots \ldots \ldots \ldots \ldots \ldots \ldots \ldots \ldots \ldots \ldots$

A. Progress on the TWOHEX Code (W. F. Walters) $\ldots \ldots \ldots \ldots \ldots \ldots \ldots$.

B. ONEDANT Code Maintenance, Improvement, and Support

(F. W. Brinkley and D. R. Marr) ................... 5

C. TWODANT Code Maintenance, Improvement, and Support

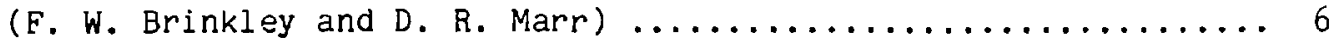

D. CHAMELEON Free-Field Loader (F. W. Brinkley) $\ldots \ldots \ldots \ldots \ldots \ldots \ldots 7$

E. Standardized INPUT and EDIT Modules (D. R. Marr) $\ldots \ldots \ldots \ldots \ldots \ldots 7$

F. REBUS Implementation ( $F$. W. Brinkley) $\ldots \ldots \ldots \ldots \ldots \ldots \ldots \ldots \ldots \ldots$

G. Time-Dependent Neutronies Transport Methods (T. R. Hill) ...... 9

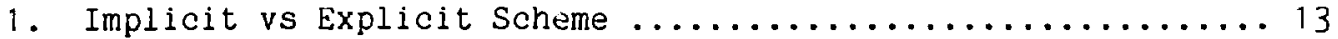

2. TIMEX Differencing vs Diamond Differencing ............ 14

3. Exponential Extrapolation vs Exponential Differencing ...... 14

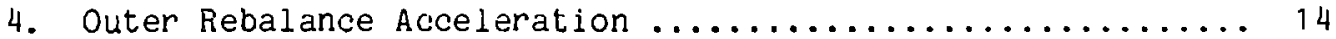

H. Comparative Transport Calculations (W. T. Urban) . . . . . . . 17

I. The Shielding Handbook (H. G. Hughes; J. M. Mack; M. Jain,

$X-5$; D. R. McCoy, $X-5$; and $B$. Wienke, $C-10) \ldots \ldots \ldots \ldots \ldots \ldots \ldots \ldots$

III. DETERMInISTIC TRANSPORT METHOdS $\ldots \ldots \ldots \ldots \ldots \ldots \ldots \ldots \ldots \ldots \ldots \ldots \ldots$

A. A New Method for the Numerical Treatment of Compton Scattering

in Radiation Diffusion Problems (E. Larsen, C. D. Levermore,

(LLNL), G. G. Pomraning (UCLA), and J. G. Sanderson) ......... 24

B. One-Dimensional, Discrete-Ordinates, Therma! Radiation

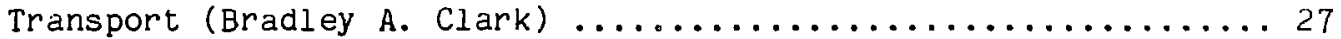

C. Thermal Radiation Transport in Two-Dimensions (R. E. Alcouffe) .. 29

D. Acceleration of High-Order $S_{N}$ Equations Using Low Order $S_{N}$

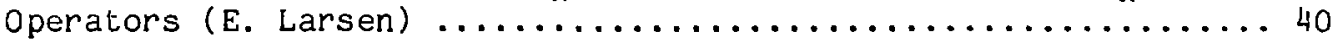

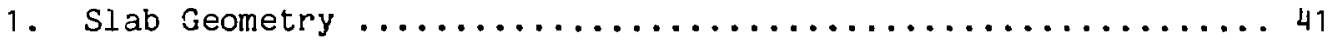

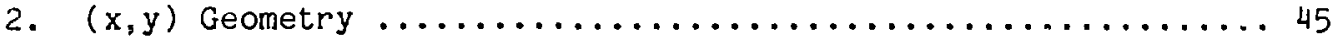

E. TRIDENT-CTR and the Streaming Matrix Hybrid Method

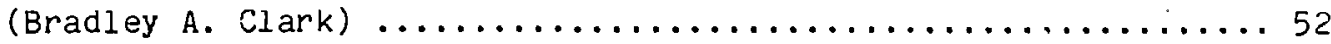

IV. MONTE CARLO RAdIATION TRANSPORT $\ldots \ldots \ldots \ldots \ldots \ldots \ldots \ldots \ldots \ldots \ldots \ldots \ldots$

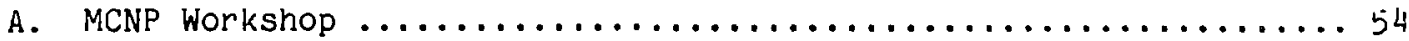

B. MCNP Maintenance, Improvement, and Support ............. 55

1. Version $3 A$ of MCNP ( $T$. N. K. Godfrey) ............... 55

2. Qualification of the MCNP Code (R. E. Seamon) ........... 56

3. Work on Version 3, MCNP (R. C. Little and R. E. Seamon) .... 56

4. Cross Sections in our Exportable Version of MCNP

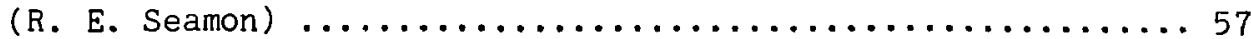

5. MCNP Activities (J. F. Briesmeister) ................ 57

6. Generalization of MCNP Standard Sources (R. G. Schrandt

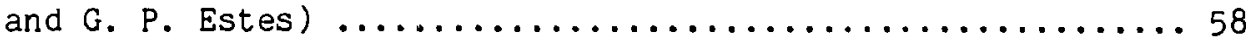


7. MCNP Patches (J. S. Henoricks) $\ldots \ldots \ldots \ldots \ldots \ldots \ldots \ldots \ldots \ldots$

a. Weight Window Generator Patch $\ldots \ldots \ldots \ldots \ldots \ldots \ldots \ldots \ldots \ldots$

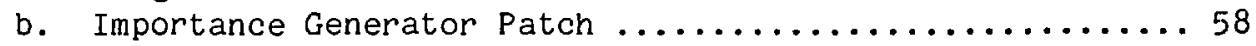

c. Exponential Transform Patch ...................... 58

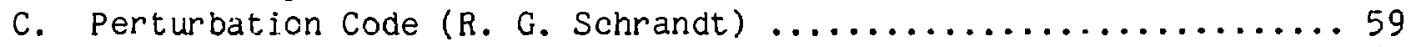

D. Angle Bias in MCNP (J. S. Hendricks) .....................6 60

E. Monte Carlo Learning/Biasing (T. E. Booth) $\ldots \ldots \ldots \ldots \ldots \ldots \ldots \ldots 6$

F. Status of CYLTRAN (H. G. Hughes and J. M. Mack) .............6 62

G. Monte Carlo Simulation of Electron/?hoton/Cerenkov Cascades in Generalized Geometry (J. M. Mack, $X-6$; and T. M. Jordan) ....664

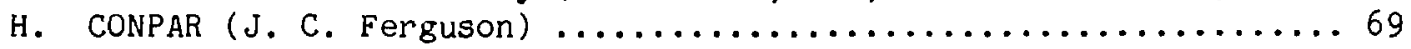

I. LWR Benchmark Coruparative Calculations (G. P. Estes and

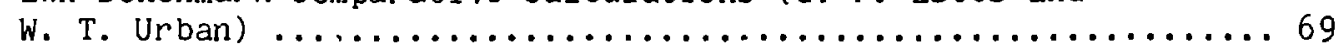

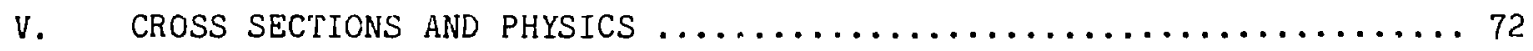

A. Biasing of Neutron-Induced Photons (R. C. Little) ...........72

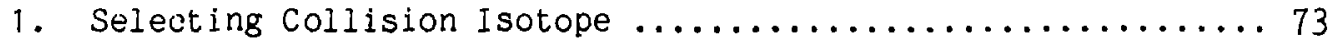

2. Sampling Photon-Production Reaction $\ldots \ldots \ldots \ldots \ldots \ldots \ldots \ldots \ldots \ldots$

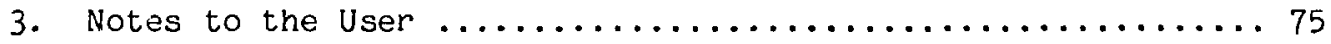

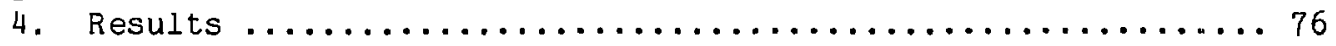

B. ENDF/B-V Revision 2 (R. C. Little and R. E. Seamon) ..........76

C. Approximate Scattering of Gamma and $X$ Rays for Monte Carlo Use when Targets are at High Temperature (but not fully ionized) and as a Function of Density (J. J. Devaney) ............... 76

D. Electron Multiple, Plural, and Single Scattering (J. J. Devaney) ............................. 78

E. Ranges of Deuterons and Tritons in 50:50 DT Plasmas at

Temperatures of 1 and $10 \mathrm{keV}$. Energy Loss per Unit Distance

(J. J. Devaney) .............................. ?9

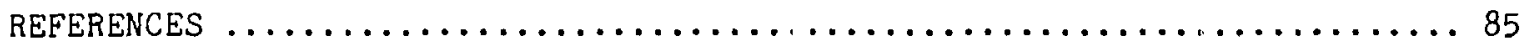




\section{RADIATION TRANSPORT}

April 1, 1983 - December 31, 1983

by

R. D. O'Dell

\section{ABSTRACT}

Research and development progress in radiation transport by the Los Alamos National Laboratory's Group X-6 for the last nine months of CY 83 is reported. Included are unclassified tasks in the areas of Fission Reactor Neutronics, Deterministic Transport Methods, Monte Carlo Radiation Transport, and Cross Sections and Physics.

\section{INTRODUCTION}

Research, development, and design analyses performed by Group $X-6$, Radiation Transport, of the Applied Theoretical Physics Division during the last nine months of CY 83 are described in this progress report. Included is the unclassified portion of programs in the Group funded by the U. S. Department of Energy (DOE). Our classified work is reported elsewhere. Some of the reported work was performed in direct support of other Laboratory groups.

This report is organized into four sections: (i) Fission Reactor Neutronics, (ii) Deterministic Transport Methods, (iii) Monte Carlo Radiation Transport, and (iv) Cross Sections and Physics. Authors of individual task reports are listed in parentheses after each task title. Authors not in Group $x-6$ have their affiliation also noted. Readers are encouraged to contact these cognizant technical personnel directly for additional information or further published results. 
Technical program management for Group $x-6$ during this reporting period was provided by William L. Thompson, Group Leader, and by Associate Group Leaders R. Arthur Forster, R. Douglas O'Dell, Dennis V. Brockway, and Patrick D. Soran.

Effective October 1, 1982, Group T-1, Transport and Reactor Theory, was joined with Group $\mathrm{X}-6$. Progress reports previously provided by Group $\mathrm{T}-1$ will no longer be published under the title of Transport and Reactor Theory but will be included in the Group $x-6$ progress reports entitled "Radiation Transport." Because of the transition in merging the groups, this progress report covers the period April 1 - December 31, 1983, a period of nine months. Hereafter, progress reports will be issued quarterly.

\section{IT. FISSION REACTOR NEUTRONICS}

The Fission Reactor Neutronics effort within Group $x-6$ is involved in the development and testing of new reactor- and shielding-oriented deterministic transport codes and methods; in existing code maintenance, improvement, and support; and in selected applications of our codes to civilian nuclear analysis problems.

In this report we describe progress in the development of our twodimensional discrete-ordinates code, TWOHEX, and our work on the ONEDANT and TWODANT discrete-ordinates codes. Also reported are improvements to our standardized free-field input data loader, CHAMELEON, and our standardized Input and Edit modules used with all our current free-standing, general-purpose, discrete-ordinates codes. A report on our attempts to implement the Argonne National Laboratory (ANL) code REBUS-3 follows. Next is provided a report on time-dependent neutronics transport methods. This is followed by a description of some comparative transport calculations using a discrete-ordinates code, a continuous energy Monte Carlo code and a multigroup Monte Carlo code. The final report in this section describes calculations performed for a shielding handbook for use by experimenters at Los Alamos.

A. Progress on the TWOHEX Code (W. F. Walters)

During the first half of this reporting period, several tasks have been completed in the development of the TWOHEX two-dimensional, equilateraltriangular mesh, discrete-ordinates code: 
1. The code has been streamlined and further vectorized in the innermost calculational routines for maximal efficiency on the CRAY-I computers,

2. The $P_{1}$ linear anisotropic scattering option has been checked out and validated,

3. Chebyshev iteration acceleration has been added to both the innerand outer-iterations to improve the convergence rate of the iterations,

4. New coding has been added to accommodate special quadrature directions encountered in 60-degree symmetric quadrature sets. The new coding improves the computational efficiency of the code.

Prior to streanlining and vectorization, the TWOHEX code was found to require about 7.5 microseconds per angular flux calculation on the Los Alamos Cray-I computers. Through a modest effort of streamlining and vectorizing the older coding, the average time per angular flux calculation was reduced to about 3.5 microseconds. This time is comparable to the $2-3$ microseconds per angular flux calculation observed in our other Los Alamos discrete-ordinates codes.

The anisotropic scattering option in TWOHEX was checked by comparing the results of a $P_{1}$ scattering calculation with those of similar runs using THREETRAN $^{1}$ (hex, $z$ ) operating in a two-dimensional mode. TWOHEX permits arbitrary anisotropic scattering order unlike THREETRAN (hex,z) which is limited to $P_{1}$ scattering.

To check the effectiveness of Chebyshev iteration acceleration, the TWOHEX code was run for a typical fast reactor core problem with all acceleration turned off and then with both inner- and outer-iteration Chebyshev acceleration turned on. For this problem it was found that the accelerated test ran nine times faster than the unaccelerated. This factor of nine is thought to be a lower limit for this class of problems. Thus, the combination of streamlining and vectorization together with the addition of Chebyshev acceleration has resulted in a speedup by a factor of nearly 20 in the TWOHEX code early in this reporting period. 
New coding was added to accomodate those discrete-ordinate directions whose projections bisect the faces of the equilateral triangular mesh. For such directions the angular flux solution equation can be simplified from the more general solution equation. By taking advantage of this simplification, TWOHEX running times were reduced a further $25 \%$.

The net result of improvements in TWOHEX enables the code to run typical discrete-ordinates calculations in running times of some 4-10 times longer than those required by the diffusion code $D I F 3 D^{2}$ at Los Alamos.

During the latter half of this reporting period, several additional features were implemented into TWOHEX in preparation for its release as a production code. Both the one-third and one-sixth core rotational boundary condition options were inplemented so that the code will now treat full core, one-third core, and one-sixth core problems. The latter two options permit the user to take advantage of the $120^{\circ}$ and $60^{\circ}$ symmetries that frequently appear in fast reactor core designs and thereby to greatly reduce the computational effort required for solution.

The latest standardized Input and Edit modules developed for the ONEDAN' 3 and TWODANT ${ }^{4}$ codes have been interfaced to the TWOHEX code so that TWOHEX now exists as a Solver module in the code package. By using the standardized Input and Edit modules, the writing of a TWOHEX User's Guide will be greatly simplified and, additionally, users of the ONEDANT and TWODANT code packages will be able to readily adjust to using TWOHEX. Additional features and capabilities were added to our standardized Input and Edit modules to accommodate using TWOHEX as a Solver module. These are discussed in Sec. II. E of this report.

The TWOHEX Solver module was modified to include a $D B^{2}$ correction for approximating the effects of $z$-dependent leakage. The bucklings, $B^{2}$, can be zone- and group-dependent and the diffusion coefficient, D, will be calculated using the transport cross section, if it is available on the cross-section library.

The focus of attention on TWOHEX in the immediate future will be the final cleanup and testing of the code and its options together with the preparation of a user's guide for the code. Upon completion of these tasks, the code will be released to the public. A target date of mid-1984 is projected for this release. 
B. ONEDANT Code Maintenance, Improvement, and Support (F. W. Brinkley and D. R. Marr)

During this reporting period several tasks were performed that can be characterized as maintenance, improvement, and support of our modular code package for one-dimensional, multigroup, discrete-ordinates neutral particle transport calculations, ONEDANT .

A new boundary condition option has been added to make a special version of ONEDANT for solar energy applications. One can now input a Bi-Direction Reflectance Distribution Function (BDRF) for use with the two-angle plane geometry capability in the code. The BDRF allows specification of the manner in which outgoing radiation is reflected back into any of the incoming directions. That is, a full matrix $Y\left(m_{i}, m_{k}\right)$ is input with entries that give the fraction of particles exiting a boundary at angle $m_{i}$ that will re-enter the boundary at angle $m_{k}$. This option has been checkbeen checked and is presently in use in atmospheric studies in which solar energy impinges on the earth's atmosphere and where the BDRF is used to represent the reflectance boundary condition at the earth's surface.

Extensive recoding of certain portions of the ONEDANT Solver module was made to improve the computational efficiency of the code on the CRAY-I computers at Los Alamos. Major recoding of the subroutines GRPDIF and INNER was performed to permit vectorization. All such coding was performed using standard FORTRAN; coding in machine langlage was avoided. Although a small amount of additional storage was required in the recoding, this was more than justified by the factor of 3 to 4 reduction in running time produced by the vectorization on certain problems. Other work to improve the computational efficiency of the code was done in the source-to-group calculation for both the diffusion and transport portions of the calculation. Coding was provided to use a change-of-pointer technique rather than the older CDC-7600 data movement technique. Cross sections were expanded to a fine mesh-point by mesh-point form instead of their coarse-mesh to coarse-mesh form to pernit vectorization.

A significant effort was devoted to initial work to convert ONEDANT rom FORTRAN-IV to FORTRAN 77 since the latter does not support the former as a subset. DATA statements of the nH form had to be converted to the "quoted" form. The readirg and writing of standard interface files as specified by the Committee on Computer Code Coordination (CCCC $)^{5}$ pose particular problems because certain records on the files contain mixes of Hollerith and real data. 
The problem results from our dependence on a single subroutine call with no CHARACTER formal parameter. The solution was to make all CHARACTER data fullword size (e.g., for a CPAY computer the CHARACTER data would be $8 H$ ). Work continued to the point that all subroutines except four were successfully compiling. These four routines contained a great deal of CHARACTER data manipulation. Work on converting these routines was deferred due to higher priority needs. All compiling was done under our FTN5 compiler which was the most FORTRAN 77-like compiler available.

During this reporting period ONEDANT was also converted to be compatible with VAX computing systems. Problems vere encountered and corrected in the existence of zero-length vectors that were not being used (when used the vectors had nonzero length). The byte-oriented nature of VAX machines presented word-boundary problems that did not exist on word-oriented computers. In particular, COMMON blocks need not begin on full-word boundary on a VAX. This makes it impossible to address one COMMON block from another using a simple index. Other conversion problems were encountered and corrected and the VAXcompatible version of ONEDANT was sent to a requesting VAX user.

C. TWODANT Code Maintenance, Improvement, and Support (F. W. Brinkley and D. R. Marr)

A final draft of the TWODANT User's Guide has been completed and sent to our Information Services editor for review. With this document the user with a minimal background in discrete-ordinates transport theory has complete input instructions for normal, full-input runs. The terminology "guide" in the title of this document is used to indicate that it is not as complete as a full reference "manual", such as the ONEDANT Inanual.3 There is no theory or background section in the Guide nor is there much discussion of the techniques involved in making partial runs. These areas, however, are somewhat generic in nature and are covered in detail in the ONEDANT manual.

The User's Guide is maintained on-line in the Los Alamos computing system in the same storage media as the executable code, so that users may access the full document from their local terminals. The User's Guide will also be published as a formal report 4 for outside users.

Conversion of TWODANT to FORTRAN 77 was undertaken as part of the ONEDANT FORTRAN 77 conversion described in Sec. II. B of this report. 
TWODANT was also converted to be VAX-computer compatible as described in Sec. II. B. A COS CRAY version of the code was also created for shipment to Sandia National Laboratory (Albuquerque) at their request.

D. CHAMELEON Free-Field Loader (F. W. Brinkley)

The CHAMELEON free-field input, loading package used in our ONEDANT and TWODANT code packages was converted to FORTRAN 77 and tested under our FIN5 compiler.

In order to make CHAMELEON more easily usable for other code developers, an effort was devoted to creating "easy-to-use" subroutines for the loading package. The only capability that is not provided by the "easy-to-use" form of CHAMELEON is the processing of stringed arrays. One code developer in our group picked up the loader and implemented it into his code with virtually no problem.

E. Standardized INPUT and EDIT Modules (D. R. Marr)

The standardized Input Module being used tor all our free-standing, discrete-ordinates codes under development was modified so that the module could be used with the triangular-mesh code TWOHEX (see Sec. II. A). Since TWOH $\mathrm{X}$ uses a mesh of equal-sized equilateral triangles, only a few pieces of input are required to specify the geometry. The array DOMAIN specifies the geometric solution domain orientation and its boundary condition. The geometric solution domain is always a parallelogram. Specifying DOMAIN= SIXTH as the input orients the parallelogram to have a $60^{\circ}$ angle at the origin (lower left-hand corner) and places $60^{\circ}$ rotational boundary conditions on the left and bottom edges of the parallelogram with vacuum conditions on the right and upper edges. This specification permits analysis of systems displaying $60^{\circ}$, or one-sixth-core, geometrical symetry. DOMAIN= THIRD orients the parallelogram to have a $120^{\circ}$ angle at the origin and places $120^{\circ}$ rotaticnal boundary conditions on the left and bottom edges of the parallelogram and vacuum conditions elsewhere. This permits the analysis of systems displaying $120^{\circ}$, or one-third-core, symetry. DOMAIN= WHOLE orients the parallelogram with a $60^{\circ}$ angle at the origin and places vacuum boundary conditions on all four edges of the parallelogram. Since the parallelogram geometric solution donain is comprised of uniformly-sized equilateral triangles, the input parameter IT is 
used to specify the total number of triangles in the $x$-dimension of the parallelogram, that is, the number of triangles in a band, and the input JT is used to specify the number of bands of triangles required to make up the parallelogram. The input parameter HEIGHT is used to specify the height of the basic equilateral triangle used as the spatial mesh cell. Finally, the zoNES array is used to indicate which triangles belong to each zone.

An additional change was made to the standardized Input Module to allow the user to input buckling, $B^{2}$, values through an input array BSQ. These buckling values are then placed on the GEODST standard interface $r i l e^{5}$ along with the other geometry input. The $B^{2}$ values can be input as a single value to be used everywhere, at the simplest extreme, or they can be specified by zone and energy group, at the other extreme.

The standardized Edit Module can now write the CCCC standard interface file RZFLUX. 5 This file contains zone-averaged multigroup fluxes. The Edit Module reads the pointwise multigroup fluxes from the RTFLUX standard interface file and the geometry information from the GEODST interface file, integrates the pointwise fluxes over each zone and forms the zone-averages. To obtain the RZFLUX file as output from the Edit Module, the user specifies RZFLUX $=1$ in the Edit input. Note that this feature can be used with an RTFLUX and GEODST file from any source and is not limited to files produced by ONEDANT Or TWODANT.

Users may now obtain power normalized values from any of the codes that interface to the Edit Module. All printed reaction rates and the RTFLUX and RZFLUX files are normalized to the power. To use, one specifies the power, in megawatts, in the new input parameter $\mathrm{POWER}=$ and the energy release (MeV) per fission in the MEVPER = input parameter. The latter parameter has a default value of $210 \mathrm{MeV} / \mathrm{f}$ ission. The fission cross section must also be available from the cross-section library. If the library is a standard ISOTXS or GRUPXS interface file, 5 the fission cross section is automatically available to the Edit Module and the user need do nothing. If the library is one of the cardimage forms, however, the user must specify which position in the crosssection table contains the fission cross section by giving the name "N-EISS" at the appropriate position in the EDNAME array found in the Block III input. 
The new RZFLUX file and power normalization options in the Edit Module were provided largely in support of performing burnup calculations for reactor core design efforts.

F. REBUS Implementation (F. W. Brinkley)

The REBUS -3 code package 6 is a package developed at Argonne National Laboratory (ANL) to perform fuel cycle analyses on fast breeder reactors. At the request of $\mathrm{T}-2$, REBUS -3 was imported and we agreed to devote a modest effort to making it operational at Los Alamos.

The first problem encountered with implementing the code involved its use of multiple versions of each of several standard interface files, e.g., RTFLUX, Version 1, RTFLUX, Version 2, etc. Our local standardized SEEK subroutine 5 for managing interface files did not allow multiple versions. We accommodated this capability by making the file name eight characters long, instead of the usual six. The first six characters define the generic file name and the last two characters the version number, e.g., RTFLUX01, RTFLUX02, etc.

The second problem encountered was that REBUS-3 requires a large number of interface files to be open at any given time. The number of such open files was about twice as many as we normally allow. This problem was quickly corrected and the ability to execute the code was achieved.

The final, and most serious, problem occurred when we attempted to execute the test problem provided with the code. Upon attempting to execute the problem in a non-overlaid form of REBUS-3, we found that the amount of $1 / 0$ time required on our computers exceeded the actual CPU computation time by at least an order of magnitude. This large I/O time rendered the code virtually unusable. Because of other higher priority programmatic needs, further work on REBUS- 3 was suspenued in Group $x-6$.

G. Time-Dependent Neutronics Transport Methods (T. R. Hill)

Since the methods for the TIMEX code 7,8 were researched by Reed, 9 little attention has been given to neutronics methods for the time-dependent transport equation. In particular, Reed did not compare the efficiency of his methods with those developed earlier for other codes.10,11 An effort was initiated this quarter to make that comparison and develop improved methods for the time-dependent transport equation. 
The time-dependent multigroup transport equation can be written

$$
V^{-1} \frac{\partial \psi}{\partial t}+(L+\Sigma) \psi=(S+F) \psi+Q
$$

where

$$
\begin{array}{ll}
\psi(\vec{r}, \vec{\Omega}, t) & =\text { vector of group angular fluxes, } \\
\text { L } & =\text { diagonal leakage operator for the appropriate geometry, } \\
\Sigma & =\text { diagonal matrix of total cross sections, } \\
S & =\text { full scatter operator, } \\
\text { F } & =\text { full fission operator, } \\
V & =\text { diagonal matrix of group speeds, and } \\
Q & \text { inhornogeneous source. }
\end{array}
$$

The explicit (more correctly, semi-implicit) method described by Reed consisted of differencing Eq. (1) (for the one group case with no inhomogeneous sources) as

$$
\frac{1}{v} \frac{\psi^{n+1}-\psi^{n}}{\Delta t}+(L+\Sigma) \psi^{n+1}=(S+F) \psi^{n},
$$

where $\psi^{n}=\psi\left(\vec{r}, \vec{\Omega}, t_{n}\right)$, the angular flux at the $n^{\prime}$ th time step. The explicit treatinent on the scatter and fission sources leads to a method without the inner and outer iterations of tine-independent codes. 12 This also can lead to a considerable loss in accuracy and force the use of a smaller than desirable (or affordable) time step. To improve the accuracy of the explicit scheme, one can use the fully implicit equation

$$
\frac{1}{v} \frac{\psi^{n+1}-\psi^{n}}{\Delta t}+(L+\Sigma) \psi^{n+1}=(S+E) \psi^{n+1}
$$

and integrate over each mesh cell and over all angles to obtain a set of finemesh rebalance equations. Using the fluxes computed from the explicit equation, Eq. (2), one can compute the rebalance factors and, upon applying these rebalance factors to the fluxes, will bring them closer to the fluxes that would result from solving the fully implicit equation, Eq. (3). 
Reed also developed a change-in-variables scheme, ter med exponential extrapolation, in which the angular flux in a tille interval is assumed to have the form

$$
\psi(t)=e^{\omega t} \hat{\psi}(t)
$$

where $\hat{\psi}(t)$ is an angular flux function that, hopefully, will vary more slowly in time and, thus, be more accurately computed for a given time step. The exponential extrapolation frequencies, both group and fine-mesh dependent but not angle-dependent, are computed from the previous time step

$$
\omega_{i, g}=\frac{1}{\Delta t} \ln \frac{\phi_{i, g}^{n}}{\phi_{i, g}^{n-1}}
$$

If the flux has achieved the $\alpha$ eigenmode distribution and is changing as $e^{\alpha t}$, the exponential extrapolation will permit, in theory, an infinitely long time step.

One of the primary advantages of the methods used in TIMEX is their unconditional stability, very important for general purpose codes that often are abused by unsophisticated users.

Considerably different methods are used in the TDA ( $\underline{T}$ ine Dependent ANISN) code. Integrating Eq. (1) over a time interval $\Delta t$, one obtains (for the one group case)

$$
\frac{1}{v} \frac{\psi^{n+1}-\psi^{n}}{\Delta t}+(L+\Sigma) \psi=(S+F) \psi
$$

where the tine interval average flux is given by

$$
\psi=\frac{1}{\Delta t} \int_{t_{n}}^{t_{n+1}} \psi(t) d t
$$


One can then use the diamond-difference approximation

$$
\psi=\frac{1}{2}\left(\psi^{n+1}+\psi^{n}\right)
$$

in Eq. (6) to obtain the approximate equation for the time-cell average flux

$$
\left(L+\Sigma+\frac{2}{v \Delta t}\right) \psi=(S+F) \psi+\frac{2}{v \Delta t} \psi^{n} .
$$

Equation (9), equivalent to a Crank-Nicholson scheme, is then solved for $\psi$, using inner and outer iterations. Upon convergence, the diamond difference relation

$$
\psi^{n+1}=2 \psi-\psi^{n}
$$

to extrapolate to the time $t_{n+1}$. As implemented in TDA, some form of fine ruesh rebalance is used to accelerate the inner iterations (only) of Eq. (9). An alternative to the diamond difference relatior, Eq. (8), available on TDA, is the exponential difference scheme. 10 This sclieme relates the timecell average flux to the edge fluxes by

$$
\begin{aligned}
& \frac{\psi^{n+1}}{\psi}=\frac{\psi}{\psi^{n}}, \text { or } \\
& \psi=\sqrt{\psi^{n+1} \psi^{n}} .
\end{aligned}
$$

Equation (11a) is designed to approximate an exponentially changing flux but, it is easy to show, does so only in the limit as $\Delta t \rightarrow 0$.

In order to avoid square roots when Eq. (11a) is inserted in Eq. (6), a weighted form of the diamond difference is used

$$
\psi=a \psi^{n+1}+(1-a) \psi^{n},
$$


where the arbitrary weight, $a$, is chosen as

$$
a=\frac{\psi^{n}}{\psi+\psi^{n}} \text {, }
$$

so that Eq. ( 11 a) is satisfied. The weight, $a$, is assumed as one-half and then computed from Eq. (13) as the inner iterations progress.

It is clea: that the TDA methods differ considerably from the TIMEX methods. In comparing the methods, a number of questions must be examined:

1. Implicit vs Explicit Scheme. The fully inplicit scheme of Eq. (3) can be used in TIMEX, with both $f$ ine mesh rebalance and exponential extrapolation, to permit larger time steps for a desired accuracy. Which is more efficient is not clear. Use of the fully implicit form, unfortunately, destroys the unconditional stability of TiMEX, at least for supercritical systems. This can be shown by rewriting Eq. (3) in the form

$$
\left(L+\Sigma+\frac{1}{v \Delta t}\right) \psi^{n+1}=(S+F) \psi^{n+1}+\frac{1}{v \Delta t} \psi^{n},
$$

and comparing with the $\alpha$ eigenvalue steady-state equation

$$
\left(L+\Sigma+\frac{\alpha}{v}\right) \psi=(S+F) \psi .
$$

The outer (fission source) iterations will diverge, unless the flux is renormalized at each outer iteration, whenever $\alpha<\alpha_{0}$, the dominant eigenvalue 13 of the system. Thus, it is obvious that the outer iterations in Eq. (14) will also diverge whenever

$$
\begin{aligned}
& \frac{1}{v \Delta t}<\frac{\alpha_{0}}{v} \text {, or } \\
& \Delta t_{\text {crit }}>\alpha_{0} .
\end{aligned}
$$


For $\Delta t \ll<\alpha_{O}$, only a few iterations will be required to converge Eq. (14). As $\Delta t \rightarrow \alpha_{0}$, the number of iterations can increase greatly, as illustrated below in a test problem, reducing the efficiency of the fully implicit scheme.

2. TIMEX Differencing vs Diamond Differencing. The time differencing of TIMEX, Eq. (2), is an order less accurate than the diamond difference (Crank-Nicholson) in TDA. Furthermore, diamond differencing in time permits a larger time step $\left(\Delta t_{\text {crit }}>2 \alpha_{0}\right.$ ) before the outer iterations diverge. Thus, diamond ditferencing has much to recommend for it. However, for systems which are decaying in time, diamond differencing can lead to negative fluxes and, thus, require a flux fixup. For systems with pulses or wave fronts moving about, it can lead to large oscillations in time, analogous to the spatial oscillations found in diamond differencing in space.

3. Exponential Extrapolation vs Exponential Differencing. The exponential difference scheme in TDA should be compared for efficiency with the exponential extrapolation of TIMEX. Because of the angular dependence of the weight, a, of $\mathrm{Eq}$. (13), the absorption term in the fine-mesh rebalance acceleration of the inners cannot be computed simply from the scalar flux. It may be, in practice, that the angular dependence of the weight is so weak that the scalar flux, rather than the angular flux, may be used to compute the weight in Eq. (13).

4. Outer Rebalance Acceleration. Both TIMEX and TDA can use fine-mesh rebalance acceleration of the inner iterations. TIMEX also has a whole-system group-collapse acceleration option on the outer iterations, but it has been found to often work pooriy. Two rebalance schemes, 13 found to work well in accelerating $\alpha$ eigenvalue calculations, can also be applied to the outers on the time dependent calculation. Both group-collapse fine-mesh rebalance and group-wise whole system rebalance have good potential.

An experimental version of TIMEX is being developed to examine these above questions and compare the efficiency of the various methods within a single code. Specifically, this experimental TIMEX now has the options:

a) Implicit treatment (i.e., iterations) on either the inners (scattering source) and/or the outers ( $f$ ission source),

b) Standard TIMEX first-order difference scheme, or diamond difference in time, or exponential differencing in time (with the weight being either based on the angular flux or the scalar flux), 
c) Outer rebalance acceleration, either group collapse whole system rebalance, group collapse fine-mesh rebalance, or group-wise whole system rebalance.

A series of test problems is being developed to compare the various methods and determine which methods work best for a particular type of problem.

One test problem is a $4.0-\mathrm{cm}$ sphere, with one group cross sections of $\Sigma_{\mathrm{a}}=0.5, v \Sigma_{\mathrm{f}}=0.8, \Sigma_{\mathrm{t}}=1.0, \Sigma_{\mathrm{S}}=0.5 \mathrm{~cm}^{-1}$, and $\mathrm{v}=10.0 \mathrm{~cm} /$ shake, with an initial distribution of the $\alpha$ eigenmode. The resulting flux is unvarying in the space and angular distribution, but increasing as $e^{\alpha t}$, where $\alpha=1.754$ gen/shake for the chosen spatial and angular discretization. The flux is calculated for $t \sum(0,10$ shakes $)$.

To compare the efficiency of the explicit vs implicit schemes, the system exponential growth is computed

$$
\omega=\frac{1}{10.0 \mathrm{sh}} \ln \frac{\bar{\phi}(\mathrm{t}=10 \mathrm{sh})}{\bar{\phi}(\mathrm{t}=0 \mathrm{sh})},
$$

where $\bar{\phi}$ is the system average flux at a given time. Clearly $\omega \rightarrow \alpha$ as the time step size is decreased.

Figure 1 shows the error in $\omega$ vs the time step size for the explicit scheme, the implicit on the inners only scheme, and the implicit on both the inners and the outers scheme. Clearly, as $\Delta t \rightarrow \Delta t_{\text {crit }}=1 / \alpha$, the outers start to diverge and the error increases without bound.

From Fig. 1, both implicit schemes are observed to produce a more accurate result for a given time step, as one would expect. However, as $\Delta t \rightarrow \Delta t_{\text {crit, }}$ the labor to do a fully implicit scheme increases greatly. By accumulating the total number of inner iterations, one can compute an "effective" time step

$$
\Delta t_{\text {eff }}=\frac{10 \text { shakes }}{\text { Total Inners }}
$$




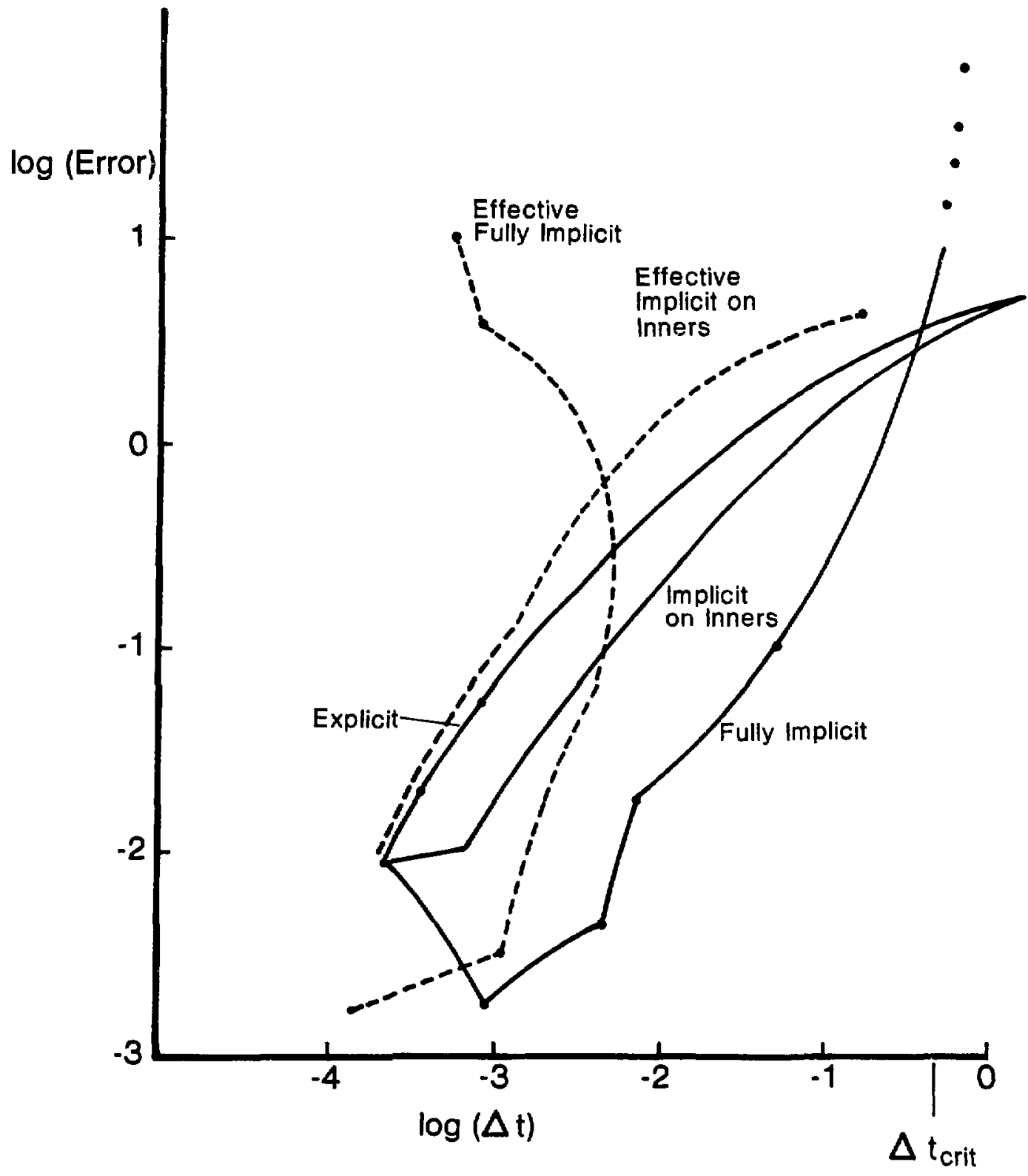

Fig. 1. Error on system exponential growth factor w vs time step size for explicit and implicit schemes. 
Which reflects the additional labor to use an implicit scheme. It is then observed from Fig. 1 that the explicit scheme is always more efficient than the implicit on inners oniy (since at least 2 inners are always required even for the smallest time step) and more efficient than the fully implicit scheme for $\Delta t>.02 \mathrm{sh}$.

H. Comparative Transport Calculations (W. T. Urban)

Effort has been, and continues to be, expended in making comparative calculations using the continuous energy and multigroup energy Monte Carlo cocies, MCNP14 and MCMC (a variant of MCNP), respectively, and the onedimensional discrete-ordinates code ONEDANT. 3 The objective of these calculations is to provide some fundamental comparisons between these codes which ultimately will be beneficial to the user community by providing some insight as to which code might be most appropriate for a particular application and also an indication of the accuracy which inight be expected. Of equal importance is the documentation of the results obtained. The following is a description of, and results for, two configurations for which calculations have been made.

The first test configuration is shown in Fig. 2 and consists of a slab $20-\mathrm{cm}$ thick, the first half of which contains a uniformly distributed monoenergetic isotropic source and the remaining half is divided into two compositionally identical regions as shown. Vacuum boundary conditions were applied
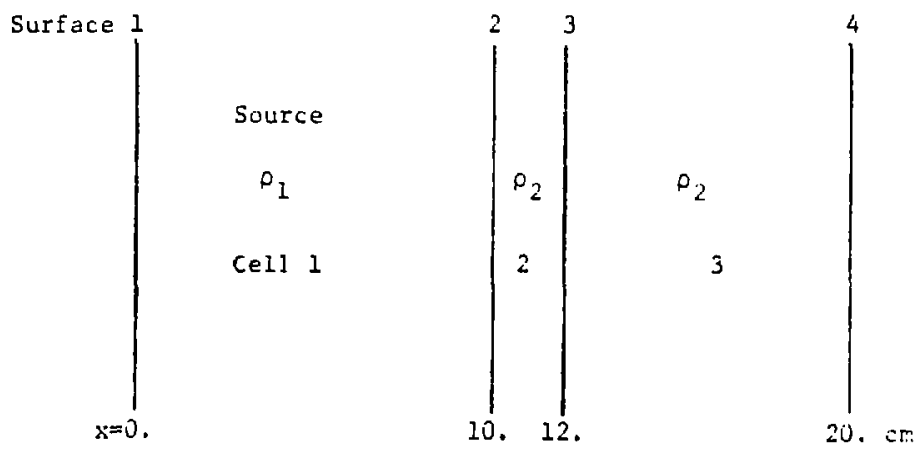

Fig. 2. One-dimensional slab test configuration. $\left\langle\rho_{1}=\frac{1}{2} \rho_{2}=0.04475 \mathrm{atom} / \mathrm{b}-\mathrm{cm}, \sigma_{a}=\sigma_{b}=1.0 \mathrm{~b}\right.$, right and left vacuum boundary conditions). 
at the left and right boundaries. In this one-group problem, the same fictitious material was used in all regions and had $\sigma_{a}=\sigma_{t}=1.0$. Number densities used for the source and remaining regions were 0.04475 and 0.0895 atoms/ b-cm, respectively. Because identical cross sections were used in each of the codes and scattering isn't allowed, cross-section differences and scattering treatments are not a factor in the comparison. Table I provides a comparison of the region averaged fluxes and the fluxes and partial currents at the bounding surfaces of the three regions. The partial currents for surfaces 2 and 3 are in the positive $(+x)$ direction. The ONEDANT surface fluxes ard the partial currents at surfaces 2 and 3 were calculated by hand from ONEDANT angular fluxes. ONEDANT results are presented for an $S_{8}$ Gauss-Legendre quadrature and $S_{8}$ and $S_{16}$ Gauss-double Legendre $\left(D P_{N}\right)$ quadrature approximations.

TABLE I

TRANSPORT RESULTS FOR TEST CONFIGURATION NO. $1^{\mathrm{a}}$

\begin{tabular}{c|c|c|c|c|c} 
& MCNP & MCMG & \multicolumn{3}{|c}{ O N E D A N T } \\
& & & $S_{8}$ & $S_{8} \mathrm{DP}_{\mathrm{N}}$ & $\mathrm{S}_{16} \mathrm{DP}_{\mathrm{N}}$ \\
& & & & & \\
\hline & & & & & \\
FLuX & & & & & \\
Cell 1 & $0.9334(0.005) \mathrm{c}$ & $0.9417(0.008)$ & 0.8978 & 0.9350 & 0.9336 \\
Cel1 2 & $0.5017(0.009)$ & $0.5014(0.015)$ & 0.5417 & 0.5026 & 0.5038 \\
Cell 3 & $0.1925(0.011)$ & $0.1928(0.017)$ & 0.1939 & 0.1917 & 0.1918 \\
& & & & & \\
Surface 1 & $0.7178(0.011)$ & $0.7089(0.018)$ & 0.7203 & 0.7165 & 0.7179 \\
Surface 2 & $0.7187(0.011)$ & $0.7152(0.018)$ & 0.7203 & 0.7165 & 0.7180 \\
Surface 3 & $0.3776(0.011)$ & $0.3763(0.0179)$ & 0.4021 & 0.3677 & 0.3756 \\
Surface 4 & $0.09175(0.017)$ & $0.09431(0.028)$ & 0.09063 & 0.09291 & 0.09249 \\
& & & & & \\
CuRRENT & & & & & \\
Surface 1 & $0.2896(0.007)$ & $0.2879(0.011)$ & 0.2993 & 0.2908 & 0.2911 \\
Surface 2 & $0.2891(0.007)$ & $0.2896(0.011)$ & 0.2993 & 0.2908 & 0.2911 \\
Surface 3 & $0.1993(0.008)$ & $0.1986(0.014)$ & 0.2023 & 0.2008 & 0.2009 \\
Surface 4 & $0.06276(0.016)$ & $0.06407(0.026)$ & 0.06338 & 0.06358 & 0.06363
\end{tabular}

asee Fig. 2 for a schematic of this configuration.

$\mathrm{b}_{\mathrm{Cell}}$ and surface averaged flux values.

Fractional error at the 10 confidence ievel. 
As expected (see Ref. 3), the $D P_{N}$ results provide better leakage fluxes for this thin slab than the same order Gauss-Legendre quadrature results. Furthermore, there is good agreement between the Monte Carlo and $D P_{N}$ discrete ordinates results.

The second test configuration, shown in Fig. 3 , is a homogeneous semiinfinite slab with a reflecting left boundary and a vacuun right boundary. The uniformly distributed isotropic source is located at $0.0 \leq x \leq 10^{-5} \mathrm{~cm}$. The extent of the problem, $x_{m}$, is infinite in the analytic model, $50 \mathrm{~cm}$ in the ONEDANT model, and $20 \mathrm{~cm}$ in the MCMG model. Calculations were performed for values of $c$ (mean number of secondaries per collision) of $U .01$ and 0.99 which correspond to neariy a black absorber and a pure scatterer, respectively. These values of $c$ were obtained through the use of fictitious cross sections which included the use of $P_{0}$ scattering cross sections. Because the value of $\Sigma_{\mathrm{t}}$ was $1.0 \mathrm{~cm}^{-1}$, each centimeter of the slab corresponds to one mean free path $(m f p)$.

Results of these calculations are presented in Table II in the form of fluxes and partial currents. It should be pointed out that the point at 0.01 mfp is a very severe test of the codes and certainly does not represent a point of general interest for practical applications. The analytic solution, obtained using Case's Method, and the 448 ONEDANT solution were provided by D. R. Marr.

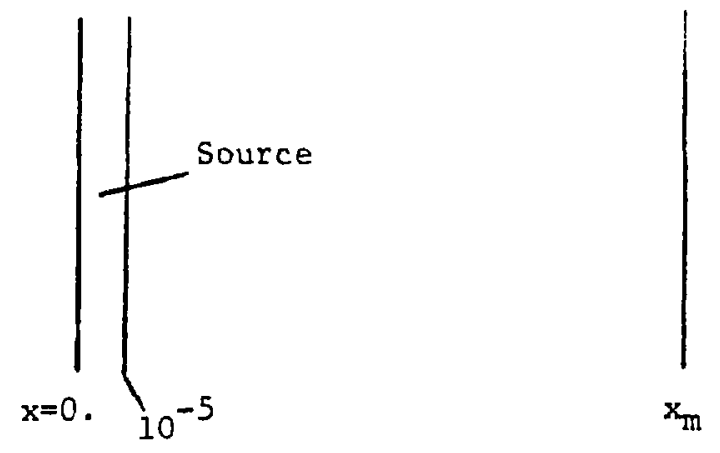

Fig. 3. One-dimensional slab test configuration. $\left(\Sigma_{t}=1.0 \mathrm{~cm}^{-1}\right.$, reflecting left boundary and vacuum right boundary). 
TABLE II

TRANSPORT RESULTS FOR TEST CONFIGURATION NO $2^{a}$

$c=0.01^{b}$

CURRENTC

\begin{tabular}{l|l|l|l|l|l|r}
$x(\mathrm{mf} p)$ & Analytic & ONEDANT & MCMG & Analytic & \multicolumn{1}{c}{ ONEDANT } & MCMG \\
\hline & & & & & & \\
0.01 & $4.762-1 \mathrm{e}$ & $4.796-1$ & $4.764-1(0.0005)^{\mathrm{f}}$ & 2.026 & 2.027 & 1.846 \\
0.1 & $3.628-1$ & $3.625-1$ & $3.625-1(0.0014)$ & $9.174-1$ & $9.172-1$ & $9.577-1(0.0032)$ \\
0.4 & $1.961-1$ & $1.961-1$ & $1.958-1(0.0028)$ & $3.550-1$ & $3.551-1$ & $3.565-1(0.0035)$ \\
1.0 & $7.505-2$ & $7.501-2$ & $7.498-2(0.0053)$ & $1.114-1$ & $1.113-1$ & $1.116-1(0.0056)$ \\
5.0 & $5.089-4$ & $5.064-4$ & $5.463-4(0.0667)$ & $5.906-4$ & $5.874-4$ & $6.397-4(0.0673)$
\end{tabular}

$c=0.99$

CURRENT

FLUX

\begin{tabular}{l|l|l|l|r|r|r}
$x(\mathrm{mf} p)$ & Analytic & ONEDANT & MCMG & ANALYTIC & \multicolumn{1}{c}{ ONEDANT } & MCMG \\
\hline & & & & & & \\
0.01 & 2.495 & 2.497 & $2.560(0.0114)$ & 10.425 & 10.419 & $10.428(0.0149)$ \\
0.1 & 2.425 & 2.425 & $2.489(0.0113)$ & 9.205 & 9.206 & $9.495(0.0145)$ \\
0.4 & 2.269 & 2.269 & $2.314(0.0109)$ & 8.262 & 8.263 & $8.414(0.0141)$ \\
1.0 & 2.028 & 2.028 & $2.076(0.0102)$ & 7.277 & 7.277 & $7.459(0.0136)$ \\
5.0 & 1.012 & 1.012 & $1.004(0.019)$ & 3.611 & 3.612 & $3.560(0.0159)$
\end{tabular}

asee Fig. 3 for a schematic of this configuration. Analytic and ONEDANT results provided by D. R. Marr.

${ }^{b} C$ is the ratio of the scattering to total cross sections.

cpartial currents in the +x direction.

dONEDANT calculations using an $\mathrm{S}_{48}$ quadrature.

$e_{4} .762-1=4.762 \times 10^{-1}$.

fFractional error at the 10 confidence level.

For the highly absorbing case, $c=0.01$, the agreement of the currents from ONEDANT and MCMG with the analytic solution is very good except for MCMG at $5.0 \mathrm{mfp}$ where the statistics are becoming larger. The MCMG flux at this same location is also high, but still within the 20 confidence interval. However, the MCMG flux at $x=0.01 \mathrm{mpf}$ is significantly lower (outside the $2 \sigma$ confidence interval) than the analytic solution but the current is in excellent agreement. It is suspected that this underprediction of the MCMG flux at $x=0.01 \mathrm{mfp}$ may be a result of the way that MCMG predicts surface flux contributions from nearly tangential (grazing) particle crossings of the surface as discussed on page 65 of Ref. 14, i.e., for cosine $\theta<0.1$ set cosine $\theta=0.05$ to compute the flux from current/cosine $\theta$. 
For the highly scattering case $(c=0.99)$ again the agreement of the ONELANT and MCMG results with the analytic results is, in general, very good. At $x=0.01 \mathrm{mfp}$ the MCMG current is not in statistical agreement at the $2 \sigma$ confidence level. However, it is comforting to see that the absolute error, $\mid$ analytic-MCMG $\mid$ /analytic $=2.6 \%$, is small. As pointed out earlier, this point so close to the source is not normally of practical interest. The good agreement of the MCMG fluxes at this same point is believed to be, in part, due to the MCMG approximation for small angle surface crossings mentioned earlier and therefore may be misleading. At $5.0 \mathrm{mfp}$ the MCMG current and flux values appear low (at points closer to the source the MCMG values are slightly higher than the analytic values) and this is partly because the MCMG model was limited to $20 \mathrm{mfp}$ whereas the ONEDANT model extended to $50 \mathrm{mfp}$ and the analytic model was infinite.

Some differences between code results have been pointed out in the above test problem comparisons; however, they tend to be small on an absolute scale. Users of the codes should consider these results to evaluate whether the cited differences in the calculated values are of practical interest in their applications. It is anticipated that additional comparative calculations will be performed using these codes and, in addition, the two-dimensional discreteordinates code, TWODANT. 4 T'wo-dimensional models and of $\mathrm{f}$-the-shelf multigroup cross-section libraries will also be considered.

I. The Shielding Handbook (H. G. Hughes; J. M. Mack; M. Jain, X-5;

D. R. McCoy, X-5; and B. Wienke, C-10)

The shielding handbook was conceived as an aid to experimenters, who often need the ability to make rough estimates of the severity of radiation backgrounds in a variety of somewhat similar situations. The original idea was to provide a body of data to describe the neutron and gamma transmissions for several representative sources and geometries, and for a number of commonly encountered shielding materials. A fairly extensive study for $14-\mathrm{MeV}$ neutron sources has now been completed, and we nonsider the project to be at a reasonable stopping place. Specifically, twelve naterials and three source/ geometry configurations were addressed. The three configurations were: (1) spherical geometry with a point isotropic source at the center of the sphere, (2) slab geometry with a collimated source normally incident on the surface of the slab, (3) slab geonetry with an isotropic source incident on the surface of the slab. The sphere radii (or slab thicknesses) were $20 \mathrm{~cm}$, 
$40 \mathrm{~cm}, 60 \mathrm{~cm}, 80 \mathrm{~cm}$, and $100 \mathrm{~cm}$. For each case, the number spectrum of transmitted particles, the energy spectrum of transmitted particles, the total material heating, the number of $(n, 2 n)$ reactions, and the number of $(n, \gamma)$ reactions were calculated. The discrete-ordinates code ONETRAN was used with ENDF/B-V multigroup cross sections for all calculations.

The material compositions, densities, and cross-section library sources are listed in Table III. As indicated in the table, a coupled 187 neutronenergy group, 24 gamma-energy group, P3 transport corrected cross-section library was used for seven materials. The standard Los Alamos coupled 30 neutron-energy group, 12 gamma-energy group cross-section library was used for five materials because a suitable 187 - 24 group library was not then available for tungsten and hydrogen.

TABLE III

MATERIALS IN THE SHIELDIHG HAMDBOOK STUDY

\begin{tabular}{|c|c|c|c|c|c|}
\hline Material & $\begin{array}{l}\text { Density } \\
\left(\mathrm{g} / \mathrm{cm}^{3}\right) \\
\end{array}$ & $\begin{array}{l}\text { Composition } \\
\text { (We1ght \%) }\end{array}$ & \multicolumn{3}{|c|}{$\begin{array}{l}\text { Cross Sections } \\
\text { (A11 ENDF/B-V) }\end{array}$} \\
\hline Copper & 8.92 & $100 \% \mathrm{Cu}$ & & 187 & - 24 \\
\hline Iron & 7.87 & $100 \% \mathrm{Fe}$ & & 187 & - 24 \\
\hline Lead & 11.34 & $100 \% \mathrm{~Pb}$ & & 187 & - 24 \\
\hline Magnet I te & 2.80 & $\begin{array}{rl}64.60 \% & \mathrm{Fe} \\
8.98 \% & \mathrm{Ti} \\
24.42 \% & 0\end{array}$ & ( $\mathrm{Fe}$ & $\begin{array}{l}187 \\
\text { self }\end{array}$ & $\begin{array}{l}24 \\
-\operatorname{shiel} \text { ded) }\end{array}$ \\
\hline Copper shot & 5.352 & $\begin{array}{l}60 \% \mathrm{Cu} \\
40 \% \text { Void }\end{array}$ & & 187 & - 24 \\
\hline Iron shot & 4.722 & $\begin{array}{l}60 \% \mathrm{Fe} \\
40 \% \text { Vold }\end{array}$ & & 187 & - 24 \\
\hline Lead shot & 6.804 & $\begin{array}{l}\text { 60\% Po } \\
40 \% \text { Void }\end{array}$ & & 187 & - 24 \\
\hline Tungsten & 19.20 & $100 \% \mathrm{~W}$ & & 30 & - 12 \\
\hline $\mathrm{CH}_{2}$ & 0.925 & $\begin{array}{l}85.63 \% \mathrm{C} \\
14.37 \% \mathrm{H}\end{array}$ & & 30 & - 12 \\
\hline $\mathrm{B}_{4} \mathrm{C}, \mathrm{CH}_{2}$ & 1.20 & $\begin{array}{l}57.6 \% \mathrm{CH}_{2} \\
30.9 \% \\
10.48 \\
(1.1 \% \\
\mathrm{C}\end{array}$ & & 30 & - 12 \\
\hline $\mathrm{Fe}, \mathrm{B}_{4} \mathrm{C}, \mathrm{CH}_{2}$ & 4.50 & $\begin{array}{rl}94.25 \% & \mathrm{Fe} \\
2.30 \% \mathrm{CH}_{2} \\
3.45 \% \mathrm{~B}_{4} \mathrm{C}\end{array}$ & & 30. & $\cdot 12$ \\
\hline $\mathrm{Cu}, \mathrm{B}_{4} \mathrm{C}, \mathrm{CH}_{2}$ & 5.00 & $\begin{aligned} 94.95 \% \mathrm{Cu} \\
2.02 \% \mathrm{CH}_{2} \\
3.03 \% \mathrm{BL}_{4} \mathrm{C}\end{aligned}$ & & 30 & - 12 \\
\hline
\end{tabular}


For the spherical geometries of $20-\mathrm{cm}$ radius, comparisons were made between the multigroup discrete-ordinates results and the continuous-energy Monte Carlo calculations using MCNP. In general, the 187 - 24 high-energy (greater than $0.1 \mathrm{MeV}$ ) neutron data and gamma data are in good agreement (typically $10 \%$ or better) with the Monte Carlo results. The $30 \cdot 12$ high-energy neutron data typically agree with the Monte Carlo results to within 15\%. However, the 30 - 12 gamma transmission data appear systematically overpredicted (by 20\% to 40\%) when compared to continuous-energy Monte Carlo. Neither library is completely satisfactory for neutron transmission below $0.1 \mathrm{MeV}$. The discrepancies for the $30 \cdot 12$ library are about twice those of the $187 \cdot 24$ library.

Potential improvements in this study include: (1) use of the $187 \cdot 24$ group library for all materials, (2) improvenents in the treatment of lowenergy neutron transmission, and (3) comparisons between the discreteordinates calculations and Monte Carlo results for the deeper penetration problems. However, our intention is to leave this study in its present form pending requests, advice, or other feedback from the users of these data.

\section{DETERMINIST IC TRANSPORT METHODS}

Our work on deterministic transport met2.ods in Group $x-6$ encompasses the development, implementation, and assessment of both analytical and numerical methods and models for the improvement and extension of deterministic transport code development.

This reporting period we report on a new method for the numerical tréatment of Compton scattering in thermal radiation diffusion problems. This is followed by reports on our progress in one-dimensional and two-dimensional thermal radiation transport using discrete-ordinates transport nethods. Next is provided a description of methods to accelerate high-order $S_{N}$ equations using low-order $S_{N}$ operators. We conclude this section with a final report on IKTLENT-CTR and the Streaning Matrix Hybrid Method. 
A. A New Method for the Numerical Treatment of Compton Scattering in Radiation Diffusion Problems (E. Larsen, C. D. Levermore (LLNL), G. C. Pomraning (UCLA), and J. G. Sanderson)

In the absence of absorption, material motion, and spatial variations, the Fokker-Planck description of time-dependent Compton scattering has the form 15

$$
\frac{\partial I}{\partial t}=\alpha \nu \frac{\partial}{\partial v}\left[\nu I+\theta\left(\frac{\partial}{\partial \nu} v I-4 I\right)+\gamma\left(\frac{I}{v}\right)^{2}\right],
$$

where $I(\nu, t)$ is the specific intensity of photons at frequency $v$ and tine $t$, and $\alpha, \theta$, and $\gamma$ are positive constants. The boundary and initial conditions for Eq. (17) are

$$
\begin{aligned}
& {\left[v I+\theta\left(\frac{\partial}{\partial v} v I-4 I\right)+\gamma\left(\frac{I}{v}\right)^{2}\right]=0 \text { for } v=0 \text { and } \infty,} \\
& I(v, 0)=I_{0}(v),
\end{aligned}
$$

where $I_{O}$ is prescribed. Equations (17) and (18) imply

$$
\frac{d}{d t} \int_{0}^{\infty} \frac{I}{v} d v \equiv \frac{d}{d t} N=0
$$

and thus $\mathrm{N}$, the total number of photons, is conserved. The steady-state ("equilibrium") solutions of Eq. (17) are

$$
I(\nu)=\frac{1}{\gamma\left(c e^{\nu / \theta}-1\right)}, \quad 1 \leq c<\infty \text {. }
$$

These are termed Böse-Einstein distributions; the special case $c=1$ is termed a Planckian distribution.

The standard method for numerically solving Eqs. (17)-(19) is due to Chang and Cooper. 16 However, this method, for nonlinear equations such as Eq. 
(17), is not positive, and for this reason it sometimes leads to code failures. In addition, it can be quite inaccurate for certain problems, and in all cases, its implementation for Eqs. (17)-(19) is not simple. We have developed a new numerical method for solving Eqs. (17)-(19) which overcomes all of these difficulties; our methed is strictly positive, it is more accurate, and it is much simpler to implement (and more efficient to run) than the Chang-Cooper method. We describe this method below.

Our method is based on rewriting Eqs. (17) and (18) in the form

$$
\begin{aligned}
& \frac{\partial I}{\partial t}=\alpha \nu \frac{\partial}{\partial \nu}\left(\nu^{2}+\gamma \frac{I}{\nu}\right)^{2} \frac{\partial}{\partial e^{\nu / \theta}} \frac{e^{\nu / \theta} I}{\nu^{3}+\gamma I}, \\
& \frac{\partial}{\partial e^{\nu / \theta}} \frac{e^{\nu / \theta} I}{\nu^{3}+\gamma I}=0 \text { for } \nu=0 \text { and } \infty \text {. }
\end{aligned}
$$

Let us introluce "center" points $0<v_{\uparrow}<v_{2}<\ldots<v_{J}<\infty$ and "edge" points $v_{j+1 / 2}$, satisfying $v_{1 / 2}=0, v_{j}<v_{j+1 / 2}<v_{j+1}$ for $1 \leq k \leq j-1$, and $v_{\mathrm{J}}<v_{\mathrm{J}+1 / 2}<\infty$. We determine the solution $I$ at the center points $v_{j}$ by the following discretization formula:

$$
\frac{I_{j}^{n+1}-I_{j}^{n}}{t^{n+1}-t^{n}}=\alpha v_{j} \frac{F_{j+\frac{1}{2}}^{n+1}-F_{j-\frac{1}{2}}^{n+1}}{v_{j+\frac{1}{2}}-v_{j-\frac{1}{2}}}, 1 \leq k \leq J \text {. }
$$

where

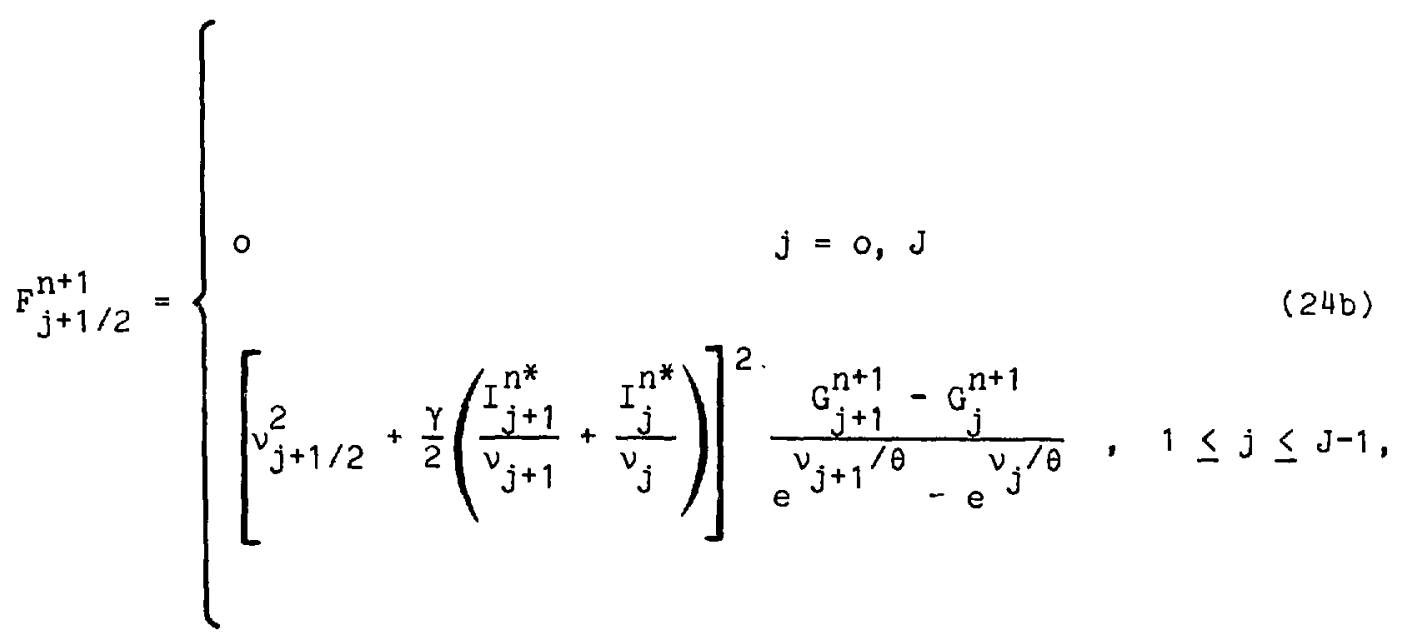


and

$$
G_{j}^{n+1}=\frac{e^{v_{j} / \theta} I_{j}^{n+1}}{v_{j}^{3}+r I_{j}^{n^{*}}}
$$

Here $I_{j}^{n}$ is the value of the intensity at $\nu_{j}$ and $t^{n}$, and (to begin) we take $I_{j}^{n^{*}}=I_{j}^{n}$. Thus, the above method is semi-inplicit, with terms of the form $\gamma I$ being evaluated at the previous time step. In practice, we have found that the following fully implicit method is more efficient: solve Eqs. (24) for (the first estimate of) $I_{j}^{n+1}$, set $I_{j}^{n^{*}}$ equal to this estimate, and then repeat the process until a suitable convergence criterion is satisfied.

The method described by Eq. (23) has the following properties:

(1) It is positive in the sense that if $u_{j}^{n} \geq 0$, then $u_{j} n^{*} \geq 0$ and $u_{j}^{n+1} \geq 0$, for both the semi-implicit and fully implicit methods. 3

(2) It is conservative, in the sense that

$$
\sum_{j=1}^{J} \frac{I_{j}^{n}}{v_{j}}\left(v_{j+1 / 2}-v_{j-\frac{1}{2}}\right)=N
$$

is independent of $\mathrm{n}$. [This is the numerical analog of Eq. (20)].

(3) The equilibrium solutions of Eqs. (24), determined by

$$
G_{j}=\text { constant , }
$$

are

$$
I_{j}=\frac{v_{j}^{3}}{r\left(c e^{v_{j} / \theta}-1\right)}, e^{-v_{1} / \theta}<c<\infty \text {. }
$$

Thus, all of the equilibrium solutions of the analytic equation, evaluated at the points $v_{j}$, are also equilibrium solutions of the numerical equations. 
(4) The full numerical method is described by Eq. (23), and is much more simple to implement than the Chang-Cooper method.

Further details on this method (including the positivity proof), together with generalizations to a wide class of linear and nonlinear FokkerPlanck operators, will be published elsewhere. 17

\section{B. One-Dimensional, Discrete-Ordinates, Thermal Radiation Transport \\ (Bradley A. Clark)}

During this reporting period, progress has been made toward a stable, accurate, and efficient scheme to solve the thermal radiation transport equation using the discrete-ordinates method. This scheme is based on a twotiered iteration strategy. The first tier is the iterative solution of the transport equation which is accelerated by the Diffusion Synthetic Acceleration (DSA) technique. The second tier is the iterative solution of the multigroup DSA equations. Now a third tier has been added; the acceleration of the multigroup DSA iteration using the multifrequency grey (MFG) technique. Experience indicates that the MFG reduces the number of multigroup DSA calculations by as much as an order of magnitude, thereby increasing the overall speed of the iterative calculations.

A new, hybrid negative flux fixup scheme has been implemented to improve the convergence rate of the transport iterations in some special cases. A detailed description of these new methods will be included in a forthcoming article. 18 Also, several new features have been added to the stand-alone code, including problem restart, SESAME tabular equation-of-state, 19 and nonanalytic opacities. 20

A test problem is used to compare various iteration strategies using the new, modified code; accuracy and computational speed are the two fundamental quantities that are studied. The problem is a $20-\mathrm{cm}$ slab of material with constant heat capacity and opacity described by the analytic relation

$$
\sigma(n, T)=\frac{27}{h \nu^{3}}\left(e^{h \nu / k T}-1\right)^{-1}
$$

The slab is divided into 20 equal-sized zones; 30 multifrequency groups are used. The initial material temperature is $1 \mathrm{eV}$. The right boundary condition $(x=20 \mathrm{~cm})$ is a $1-\mathrm{eV}$ blackbody source; the left boundary condition is a 
blackbody that varies from $1 \mathrm{eV}$ at zero time to $1 \mathrm{keV}$ at 0.1 nanoseconds, after $0.1 \mathrm{~ns}$ the temperature remains at $1 \mathrm{keV}$. The initial time step is $10^{-12}$ seconds. While the problem is running, the time step is controlled so that the maximum change in temperature in any zone during each time step is approximately constant.

A base case iteration strategy was chosen to provide the most accurate results for comparison with later runs. This strategy included fully iterated transport with DSA and MFG acceleration. In addition, the opacities are iterated to convergence at each time step using paraneter iteration. 18 Convergence is attained when the change between pointwise angular fluxes is less than $1 \%$. Time steps are controlled to maintain a maximum temperature change of $5 \%$ in each time step. (Results from other runs using $1 \%$ temperature change, 200 zones, and 40 groups were virtually identical to the base case, indicating its acceptability as an accurate solution.)

The first step in optimizing the iteration strategy is to eliminate the parameter iteration and use opacities calculated from the temperatures obtained after the previous time step. This simplification is acceptable as long as accuracy is not overly sacrificed. The results indicate that if the temperature change is limited to less than 10-25\%, the material temperatures are within a few percent of the base case solution. If the temperature change is allowed to go as high as $100 \%$, the speed of the wave traveling through the material is reduced to about half of the base case speed.

Eliminating the parameter iteration has the benefit of increasing the speed of the calculation. In this case, it is 10 times faster than the base case. Since this routine uses an analytic opacity model, which is evaluated much faster than nonanalytic or tabular models, the increase in speed noted here will be even greater when more complicated opacity models are used in real problems.

The second step in obtaining a faster iteration strategy is limiting the number of iterations allowed in each time step. Limiting to two transport iterations per time step and five DSA iterations per transport iteration (including MFG acceleration of the DSA) calculates material temperatures that are within a few percent of those obtained using the full iteration strategy. Limiting the iterations yields an additional factor of two speed-up over the base case. 
whether it is more efficient to iterate or simply take smaller time steps is a long-standing question. In this case, to obtain accurate results without iteration requires reducing the temperature change criterion from $25 \%$ to less than $0.5 \%$. More significantly, the uniterated run requires 20 times more computer time than the linited iteration run. If more complicated opacity models were used, the advantages of limited iteration are even greater. The studies reported here have shown that many of the base case iteration schemes are needed only for very large time steps. If time steps are limited by the temperature change criterion, it is better to choose a 25\% temperature change and use limited iteration than to choose a smaller criterion and eliminate iteration. However, in radiation hydrodynamics calculations there are situtations when time steps are limited by considerations other than the temperature change criterion (hydrodynamics, other physical processes that are changing rapidly, etc.). In those situations iteration may be unnecessary. The advantage of the current iteration method is that all cases can be solved optimally when the iteration strategy can be adjusted to suit the problem being solved.

In spite of the excellent results obtained so far, a single problem remains; in some situations the DSA calculation will produce negative intermediate results. When the negative values are used in the MFG to obtain grey constants, nonphysical fluxes may be calculated. Usually, these cases produce negative absorption rates that eventually lead to negative temperatures and catastrophic failure of the iteration. Future work will concentrate on eliminating this problem.

C. Thermal Radiation Transport in Two-Dimensions (R. E. Alcouffe)

A two-dimensional code is being developed from the TWODANT code to solve the stationary radiation hydrodynamics equations in two-dimensional ( $x, y$ ) and $(r, z)$ geometries. This is based upon techniques developed for the one-dimensional radiation hydrodynamics problem which is discussed in the preceding section. The present status of the two-dimensional code is that the steady state distribution for the temperature and the multigroup radiation intensity is obtained in the $S_{N}$ transport approximation. Thus, we demonstrate the feasibility of performing these calculations. What remains to be done are comparison calculations verifying the solution obtained and assessing the efficiency of the calculational method. The capability to perform time-dependent 
calculations is also to be added in order to assess the performance of the method in this regime. In the following we present the details of the twodimensional implementation and some calculational examples of the code's performance.

The general outline of the method for solving the radiation hydrodynamics problem for stationary media is given in Ref. 18. In this section we sketch the steps that we have used to implement the solution method in twodimensional geometry for the diamond-differenced $S_{N}$ equations. The problem to be solved is described by the following equations:

$$
\begin{gathered}
\frac{1}{c} \frac{\partial I}{\partial t}+\underline{\Omega} \cdot \nabla I(\underline{r}, v, \underline{\Omega}, t)+\sigma(\underline{r}, v, T) I(\underline{r}, v, \underline{\Omega}, t) \\
=\sigma(\underline{r}, v, T) B(v, T)+Q\left(\underline{r}, v, T_{0}\right),
\end{gathered}
$$

and

$$
\begin{aligned}
\frac{\partial u_{e}}{\partial t}= & \beta(\underline{r}, T)\left[\int_{0}^{\infty} \sigma\left(\underline{r}, v^{\prime}, T\right) I_{0}\left(\underline{r}, v^{\prime}, t\right) d v^{\prime}\right. \\
& \left.-c \sigma_{p}(\underline{r}, T) u_{e}(\underline{r}, t)+w(\underline{r}, t)\right],
\end{aligned}
$$

where

$$
\begin{aligned}
& B(v, T)=\frac{2 h v^{3}}{c^{2}}\left(e^{h v / k T}-1\right)^{-1}, \\
& u_{e}(\underline{r}, t)=a T^{4}(\underline{r}, t), \\
& I_{0}(\underline{r}, v, t)=\int_{4 \pi} I(\underline{r}, v, \underline{\Omega}, t) \underline{d} \underline{\Omega},
\end{aligned}
$$




$$
\sigma_{p}(\underline{r}, T)=\int_{0}^{\infty} B(v, T) \sigma(\underline{r}, v, T) d v / \int_{0}^{\infty} B(v, T) d v .
$$

In the above $I(\underline{r}, v, \underline{\Omega}, t)$ is the specific intensity of radiation defined such that $\underline{n} \cdot \underline{\Omega} I(\underline{r}, \nu, \underline{\Omega}, t) d \nu d \underline{\Omega}$ dS $d t$ is the amount of radiation energy transported across a surface element dS with normal $\underline{n}$, at space point $\underline{r}$, in the frequency range $d v$ about $v$, in the solid angle $d \underline{\Omega}$ about $\underline{\Omega}$, in the time interval dt about t. Also $T(\underline{r}, t)$ is the material temperature at $\underline{r}$ and $t$, and $\sigma(\underline{r}, v, T)$ is the macroscopic absorption cross section (density times opacity) defined such that if the material is at temperature $T$, then the probability that a photon at $(\underline{r}, v)$ will be absorbed in a distance ds is ods. The function $\beta(\underline{r}, T)$ is related to the specific heat of the material and is defined through the relation

$$
\frac{1}{B}=\frac{\partial u_{m}}{\partial u_{e}}
$$

where $u_{m}$ is the material energy density and $u_{e}$ is as defined in Eq. (28b). $W(\underline{r}, t)$ reuresents any extraneous source to the energy equation. To arrive at the appropriate form for the transport problem, we time-difference Eq. (26) and (27) and combine them to obtain

$$
\begin{aligned}
& \underline{\Omega} \cdot \nabla I^{n+1}(\underline{r}, \underline{\Omega}, v)+\left[\sigma\left(\underline{r}, v, T^{*}\right)+\tau^{n}\right] I^{n+1}(\underline{r}, \underline{\Omega}, v) \\
&=\frac{x\left(\underline{r}, v, T^{*}\right)}{4 \pi\left[1+\tau^{n} \alpha\left(\underline{r}, T^{n^{*}}\right)\right]}\left[\int_{0}^{\infty} \sigma\left(\underline{r}, v^{\prime}, T^{*}\right) I_{0}^{n+1}\left(\underline{r}, v^{\prime}\right) d v^{\prime}\right. \\
&\left.+W^{n}(\underline{r})+\frac{u_{e}^{n}(\underline{r})}{\Delta t^{n}{ }_{B}\left(\underline{r}, T^{n *}\right)}\right]+\tau^{n} I^{n}(\underline{r}, v, \underline{\Omega}) \\
&+Q^{n+1}\left(\underline{r}, v, T_{0}\right)
\end{aligned}
$$




$$
\begin{aligned}
u_{e}^{n+1}(\underline{r})= & \left\{\sigma_{p}\left(\underline{r}, T^{*}\right)\left[1+\tau^{n} \alpha\left(\underline{r}, T^{n^{*}}\right)\right]\right\}^{-1}\left[\int_{0}^{\infty} \sigma\left(\underline{r}, v, T^{*}\right) I_{0}^{n+1}\left(\underline{r}, v^{\prime}\right) d v^{\prime}\right. \\
& \left.+w^{n}(\underline{r})\right]+\frac{u_{e}^{n}(\underline{r})}{\Delta t^{n}\left(\underline{r}, T^{n^{*}}\right)} \\
T^{n+1}(\underline{r})= & \frac{1}{a} u_{e}^{n+1}(\underline{r}){ }^{1 / 4}
\end{aligned}
$$

where

$$
\begin{aligned}
& \tau^{n}=\frac{1}{c \Delta t^{n}}, \\
& \alpha\left(\underline{r}, T^{n^{*}}\right)=\left[a_{p}\left(\underline{r}, T^{n^{*}}\right) B\left(\underline{r}, T^{n^{*}}\right)\right]^{-1}, \\
& x\left(\underline{r}, u m T^{n^{*}}\right)=\frac{\sigma\left(\underline{r}, v, T^{n^{*}}\right) B\left(v, T^{n^{*}}\right)}{\iota_{p}\left(\underline{r}, T^{n^{*}}\right) \int_{0}^{\infty} B\left(v^{\prime}, T^{n^{*}}\right) d v^{\prime}},
\end{aligned}
$$

and $\mathrm{T}^{*}(\underline{r})$ is the temperature evaluated at some time $t^{n} \leq t^{n^{*}} \leq t^{n+1}$.

The solution process for our problem proceeds as follows: Eq. (30) is solved for the intensity at time $t^{n+1}$ given parameters evaluated at temperature $\mathrm{T}^{\mathrm{n}}$; then Eq. (31) is solved for $\mathrm{u}_{e}^{\mathrm{n}+1}$ and a new temperature obtained from Eq. (32). With this new temperature, the parameters of Eqs. (30) and (31) may be reevaluated and the entire solution process repeated. Such a parameter iteration may then be continued until the temperature is converged to some desired criterion. This solution process, including the parameter iteration, is stable for any size time step and has been showil to converge in oredimensional cases. ${ }^{18}$ We note that in order to solve Eq. (30) a source iteration procedure, described in Ref. 18, is invoked which must, in general, be accelerated. The solution procedure witr acceleration developed for the twodimensional case will be shown below for the special case of an infinite time step, that is, for $\tau^{n}=0$. 
The discrete-ordinates, two-dimensional diamond-differenced radiation hydrodynamics equation for $\tau^{n}=0$ is written as

$$
\begin{aligned}
& \mu_{m}\left(A_{i+\frac{1}{2}} I_{i+\frac{1}{2}}^{\ell+\frac{1}{2}}-A_{i-\frac{1}{2}} I_{i-\frac{1}{2}}^{\ell+\frac{1}{2}}\right)+\left(B_{m}-\mu_{m}\right)\left(A_{i+\frac{1}{2}}-A_{i-\frac{1}{2}}\right) I^{\ell+\frac{1}{2}} \\
& +\mu_{m} B_{i j}\left(I_{j+\frac{1}{2}}^{\ell+\frac{1}{2}}-I_{j-\frac{1}{2}}^{\ell+\frac{1}{2}}\right)+\left(\sigma_{g}^{*} V\right)_{i j} I^{\ell+\frac{1}{2}} \\
& =x_{g i j}^{*} \sum_{g^{\prime}=1}^{G}\left(\sigma_{g}^{*}, V\right)_{i j} I_{O g^{\prime}}^{\ell}+B_{m}\left(A_{i+\frac{1}{2}}-A_{i-\frac{1}{2}}\right)^{\ell+\frac{1}{2}}+\left(Q_{g} V\right)_{i j}
\end{aligned}
$$

and

$$
I_{m}^{l+\frac{1}{2}}=\frac{1}{2}\left(I_{m+\frac{1}{2}}^{l+\frac{1}{2}}+I_{m-\frac{1}{2}}^{\ell+\frac{1}{2}}\right)
$$

for $1 \leq m \leq M$ and $1 \leq B \leq G$, and

$$
u_{e i g}=\frac{1}{c \sigma_{p i j}^{*}} \sum_{g^{\prime}=1}^{G}\left(\sigma_{g}^{*} v\right)_{i j} I_{o g},
$$

and

$$
\mathrm{T}_{i j}=\left(\frac{1}{\mathrm{a}} \mathrm{u}_{e i j}\right)^{\frac{1}{4}}
$$

where * denotes parameters evaluated at some temperature $T^{*}$ and $l$ is an iteration index. Also, in the above equations cell-centered indices, e.8., i, $j$, $\mathrm{m}, \mathrm{g}$, have been omitted when clarity is not impaired. Equations $(33)-(36)$ assume that $\tau^{\mathbf{n}}=0, i . e ., \Delta t^{n}=\infty$ and that the variables have been discretized in angle, space, and frequency. Eq. (33) is a standard form of the discreteordinates equations assuming diamond differencing in angle per Eq. (34); and we make the further approximations of diamond differencing in the spatial dimensions, 


$$
\begin{aligned}
& I_{i}^{\ell+\frac{1}{2}}=\frac{1}{2}\left(I_{i+\frac{1}{2}}^{\ell+\frac{1}{2}}+I_{i-\frac{1}{2}}^{\ell+\frac{1}{2}}\right), \\
& I_{j}^{\ell+\frac{1}{2}}=\frac{1}{2}\left(I_{j+\frac{1}{2}}^{\ell+\frac{1}{2}}+I_{j-\frac{1}{2}}^{\ell+\frac{1}{2}}\right),
\end{aligned}
$$

Equations (37) are further modified if the edge values extrapolate to negative values upon solving Eq. (33) in that we use a set-to-zero fixup to ensure that the solution to (33) is nonnegative.

We note that Eq. (33) is solved by iteration and for radiation transport problens it is vital that some sort of diffusion synthetic acceleration be used. Eollowing Refs. 18 and 21, we propose the following nonlinear acceleration method for Eq. (33):

$$
\begin{aligned}
& -F L_{g, i+1, j+\frac{1}{2}}^{\ell+\frac{1}{2}}\left(I_{O g, i+3 / 2, j+\frac{1}{2}}^{\ell+1}-I_{O g, i+\frac{1}{2}, j+\frac{1}{2}}^{\ell+1}\right) \\
& +F L_{g, i, j+\frac{1}{2}}^{\ell+\frac{1}{2}}\left(I_{O g, i+\frac{1}{2}, j+\frac{1}{2}}^{\ell+1}-I_{O g, i-\frac{1}{2}, j+\frac{1}{2}}^{\ell+1}\right) \\
& -F D_{g, i+\frac{1}{2}, j+1}^{l+\frac{1}{2}}\left(I_{O g, i+\frac{1}{2}, j+3 / 2}^{\ell+1}-I_{O g, i+\frac{1}{2}, j+\frac{1}{2}}^{l+1}\right) \\
& +F D_{g, i+\frac{1}{2}, j}^{\ell+\frac{1}{2}}\left(I_{O g, i+\frac{1}{2}, j+\frac{1}{2}}^{\ell+1}-I_{O g, i+\frac{1}{2}, j-\frac{1}{2}}^{\ell+1}\right) \\
& +\left(\sigma_{g}^{*} V\right)_{i+\frac{1}{2}, j+\frac{1}{2}}^{l+\frac{1}{2}} I_{O g, i+\frac{1}{2}, j+\frac{1}{2}}^{l+1}=x_{g, i+\frac{1}{2}, j+\frac{1}{2}}^{* l+1} \Phi_{i+\frac{1}{2}, j+\frac{1}{2}}^{l+1} \\
& +\left(Q_{g} V\right)_{i+\frac{1}{2}, j+\frac{1}{2}}, g=1, \ldots, G,
\end{aligned}
$$

where

$$
\mathrm{FL}_{\mathrm{B}, i, j+\frac{1}{2}}^{\ell+\frac{1}{2}}=-\frac{\left(A_{1+\frac{1}{2}, j} J_{1+\frac{1}{2}, J}+A_{1-\frac{1}{2}, J} J_{1-\frac{1}{2}, j}+A_{1+\frac{1}{2}, j+1} J_{1+\frac{1}{2}, j+1}+A_{i-\frac{1}{2}, J+1} J_{1-\frac{1}{2}, j+1}\right)^{l+\frac{1}{2}}}{I_{0,1+\frac{1}{2}, J+\frac{1}{2}}^{l+\frac{1}{2}}-I_{0,1-\frac{1}{2}, J+\frac{1}{2}}^{l+\frac{1}{2}}}
$$




$$
F D_{g, i+\frac{1}{2}, j}^{l+\frac{1}{2}}=-\frac{B_{i, j}\left(K_{i, j+\frac{1}{2}}+K_{i, j-\frac{1}{2}}\right)^{l+\frac{1}{2}}+B_{i+1, j}\left(K_{i+1, j+\frac{1}{2}}+K_{i+1, j-\frac{1}{2}}\right)^{l+\frac{1}{2}}}{I_{0, i+\frac{1}{2}, j+\frac{1}{2}}^{l+\frac{1}{2}}-I_{0, i+\frac{1}{2}, j-\frac{1}{2}}^{l+\frac{1}{2}}}
$$

$$
\begin{aligned}
& J_{i+\frac{1}{2}, j}^{l+\frac{1}{2}}=\sum_{m=1}^{M} w_{m} m_{m}^{I} m, i+\frac{1}{2}, j \\
& k_{i, j+\frac{1}{2}}^{\ell+\frac{1}{2}}=\sum_{m=1}^{M} w_{m}{ }^{n} I_{n, i, j+\frac{1}{2}}^{l+\frac{1}{2}},
\end{aligned}
$$

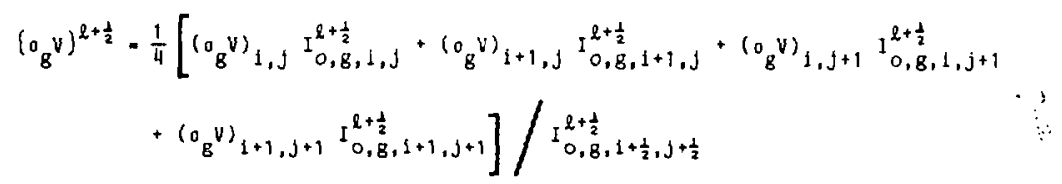

$$
\begin{aligned}
& x_{g, i+\frac{1}{2}, j+\frac{1}{2}}^{*} \Phi_{i+\frac{1}{2}, j+\frac{1}{2}}^{\ell+1}=\frac{1}{4}\left[x_{g, i, j} \sum_{g^{\prime}=1}\left(\sigma_{B^{\prime}}, V\right)_{i, j} I_{0, g^{\prime}, i, j}^{\ell+1}\right. \\
& +x_{g, i+1, j} \sum_{g^{\prime}+1}^{G}\left(\sigma_{g}, V\right)_{i+1, j^{I+1}}^{\ell, g^{\prime}, i+1, j} \\
& +x_{g, i, j+1} \sum_{g^{\prime}=1}^{G}\left(\sigma_{g^{\prime}}, V\right)_{i, j+1} I_{o, g^{\prime}, i, j+1}^{l+1} \\
& \left.+x_{g, i+1, j+1} \sum_{g^{\prime}=1}^{G}\left(\sigma_{B^{V}} V\right)_{i+1, j+1} I_{0, g^{\prime}, i+1, j+1}^{l+1}\right] \text {, }
\end{aligned}
$$


and

$$
\left(Q_{g} V\right)_{i+\frac{1}{2}, j+\frac{1}{2}}=\frac{1}{4}\left[\left(Q_{g} V\right)_{1, j}+\left(Q_{g} V\right)_{i+1, j}+\left(Q_{g} V\right)_{i, j+1}+\left(Q_{g} V\right)_{i+1, j+1}\right]
$$

We classify Eq. (38) as the diffusion correction method for the diffusion acceleration of the transport equation. It is constructed so that upon convergence, Eq. (38) and Eq. (33) will have the same scalar solution, $I_{0, g, i, j}^{\ell}$, for $\ell \rightarrow \infty$. This is the standard method encoded in the TWODANT code 4 and is satisfactory for many applications. We note that we have chosen a five-point differencing form of the acceleration equation for ease of solution with conventional diffusion equation solvers. The iteration is nonlinear and, hence, is not amenable to analysis, but it seems to perform as well as the linear method performance in model problems. 22

It is seen that the solution of $\mathrm{Eq} .(38)$ is on the mesh vertices whereas the source for Eq. (33) is a mesh-centered quantity. At present we use the rollowing simple relationship to go from mesh vertex quantities to meshcentered quantities.

$$
I_{0,8, i, j}^{l+1}=I_{0,8, i, j}^{l+\frac{1}{2}} \frac{\left(I_{0,8,1+\frac{1}{2}}+I_{0,8, l+3 / 2, j+\frac{1}{2}}+I_{0,8,1+\frac{1}{2}, j+3 / 2}+I_{0, g, 1+3 / 2, j+3 / 2}\right)^{l+1}}{\left(I_{0,8,1+\frac{1}{2}, j+\frac{1}{2}}+I_{0,8, i+3 / 2, j+\frac{1}{2}}+I_{0,8,1+\frac{1}{2}, j+3 / 2}+I_{0,8,1+3 / 2, j+3 / 2}\right)^{l+\frac{1}{2}}} .
$$

The procedure seems to suffice for diamond differencing of the transport equation but can be seneralized to include the effects of flux fixup. 18

Now Eq. (38) is a multigroup diffusion equation which must itself be solved by source $i$ teration since $\Phi_{i+\frac{1}{2}, j+\frac{1}{2}}$ is a function of the solution $I_{\circ, 8, i+\frac{1}{2}, j+\frac{1}{2}}$ summed over all groups. Following Ref. 18 we derive a one-group equation which will accelerate this source iteration procedure for Eq. (38). We rewrite Eq. (38) here with a diffusion iteration index, $k$ :

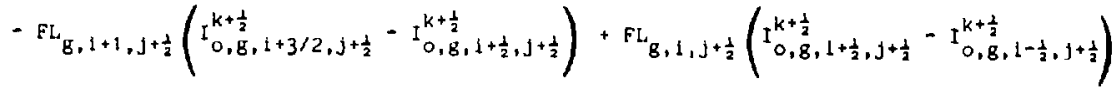

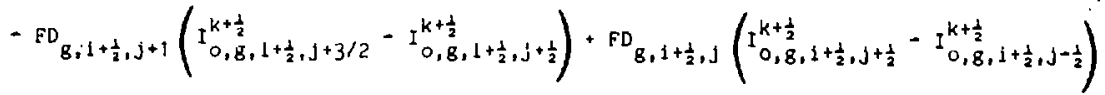

$$
\begin{aligned}
& \text { + }\left(0_{8}^{*} V\right)_{1+\frac{1}{2}, j+\frac{1}{2}}^{k} I_{0,8,1+\frac{1}{2}, j+\frac{1}{2}}^{k+\frac{1}{2}}=x_{8,1+\frac{1}{2}, j+\frac{1}{2}}^{* k} \phi_{i+\frac{1}{2}, j+\frac{1}{2}}^{k}+\left(Q_{g} V\right)_{1+\frac{1}{2}, j+\frac{1}{2}} \text {. }
\end{aligned}
$$


where

$$
\begin{aligned}
& \left(0_{g}^{*} v\right)_{0+\frac{1}{2}, j+\frac{1}{2}}^{k}=\frac{1}{4}\left[\left(0_{8}^{* v)_{1 j}} I_{0,8,1, j}^{k}+\left(0_{g}^{*} * v\right)_{1+1, j} I_{0,8,1+1, g}^{k}+\left(0_{g}^{*} *\right)_{1, j+1} I_{0,8,1, j+1}^{k}\right.\right. \\
& \left.+\left(a_{g} * V\right)_{1+1, j+1} I_{0,8,1+1, j+1}^{k}\right] / I_{0,8,1+\frac{1}{2}, j+\frac{1}{2}}^{k} \text {. } \\
& x_{g, i+\frac{1}{2}, j+\frac{1}{2}}^{k}-\frac{x_{g, i, j} \Phi_{1 j}^{k}{ }^{k} x_{g, i+1, j} \Phi_{i+1, j}^{k}+x_{g, i, j+1} \Phi_{i, j+1}^{k}+x_{g, i+1, j+1} \Phi_{i+1, j+1}^{k}}{\Phi_{i, j}^{k}+\phi_{1+1, j}^{k}+\phi_{1, j+1}^{k}+\phi_{1+1, j+1}^{k}} . \\
& \Phi_{i+\frac{1}{2}, j+\frac{1}{2}}^{k}=\frac{1}{4} \Phi_{i, j}^{k}+\phi_{i+1, j}^{k}+\Phi_{i, j+1}^{k}+\Phi_{i+1, j+1}^{k} .
\end{aligned}
$$

The removal term in Eq. (41) is made nonlinear so that the one-group form will be simplified in that absorption and emission is balanced at each step of the iteration. In order to derive the acceleration equation for Eq. (41), we replace

$$
I_{g, i+\frac{1}{2}, j+\frac{1}{2}}^{k+\frac{1}{2}} \rightarrow \frac{I_{g, i+\frac{1}{2}, j+\frac{1}{2}}^{k+\frac{1}{2}} I_{O, j+\frac{1}{2}, j+\frac{1}{2}}^{k+1}}{\sum_{g^{\prime}} I_{g^{\prime}, i+\frac{1}{2}, j+\frac{1}{2}}^{k+\frac{1}{2}}}
$$

for $i=1,2, \ldots$, IT; $j=1,2, \ldots$, JT; $g=1,2, \ldots, G$.

Making the substitution Eq. (43) into Eq. (41) and summing over all groups yields

$$
\begin{aligned}
& -F R_{i, j+\frac{1}{2}}^{k+\frac{1}{2}} I_{O, i+3 / 2, j+\frac{1}{2}}^{k+1}-F L_{i, j+\frac{1}{2}}^{k+\frac{1}{2}} I_{O, i-\frac{1}{2}, j+\frac{1}{2}}^{k+1}-F U_{i+\frac{1}{2}, j}^{k+\frac{1}{2}} I_{O, i+\frac{1}{2}, j+3 / 2}^{k+1} \\
& -F D_{i+\frac{1}{2}, j}^{k+\frac{1}{2}} I_{O, i+\frac{1}{2}, j-\frac{1}{2}}^{k+1}+A{ }_{i+\frac{1}{2}, j+\frac{1}{2}}^{k+\frac{1}{2}} I_{O, i+\frac{1}{2}, j+\frac{1}{2}}^{k+1}=(Q V)_{i+\frac{1}{2}, j+\frac{1}{2}}
\end{aligned}
$$


for $i=0, i, \ldots$, IT and $j=0,1, \ldots, \mathrm{JT}$,

where

$F R_{i, j+\frac{1}{2}}^{k+\frac{1}{2}}=\frac{\sum_{g=1}^{G} F L_{g, i+1, j+\frac{1}{2}} I_{0, g, i+3 / 2, j+\frac{1}{2}}^{k+\frac{1}{2}}}{I_{0, i+3 / 2, j+\frac{1}{2}}^{k+\frac{1}{2}}}$,

$F L_{i, j+\frac{1}{2}}^{k+\frac{1}{2}}=\frac{\sum_{g=1}^{G} F L_{g, i, j+\frac{1}{2}} I_{o, g, i-\frac{1}{2} \cdot k+\frac{1}{2}}^{k+\frac{1}{2}}}{I_{0, i-\frac{1}{2}, j+\frac{1}{2}}^{k+\frac{1}{2}}}$,

$F U_{i+\frac{1}{2}, j}^{k+\frac{1}{2}}=\frac{\sum_{g=1}^{G} F D_{g, i+\frac{1}{2}, j+1} I_{0, g, i+\frac{1}{2}, j+3 / 2}^{k+\frac{1}{2}}}{I_{0, i+\frac{1}{2}, j+3 / 2}^{k+\frac{1}{2}}}$,

$F D_{i+\frac{1}{2}, j}^{k+\frac{1}{2}}=\frac{\sum_{g=1}^{G} F D_{g, i+\frac{1}{2}, j} I_{0,8, i+\frac{1}{2}, j-\frac{1}{2}}^{k+\frac{1}{2}}}{I_{0, i+\frac{1}{2}, j-\frac{1}{2}}^{k+\frac{1}{2}}}$.

$A_{i+\frac{1}{2}, j+\frac{1}{2}}^{k+\frac{1}{2}}=\frac{\sum_{g=1}^{C}\left(F L_{g, 1+1, j+\frac{1}{2}}+F L_{g, 1, j+\frac{1}{2}}+F L_{g, i+\frac{1}{2}, j+1}+F L_{g, i+\frac{1}{2}, j}\right) I_{0, B, i+\frac{1}{2}, j+\frac{1}{2}}^{k+\frac{1}{2}}}{I_{0, j+\frac{1}{2}, J+\frac{1}{2}}^{k+\frac{1}{2}}}$.

and 


$$
I_{0, i+\frac{1}{2}, j+\frac{1}{2}}^{K+\frac{1}{2}}=\sum_{g=1}^{G} I_{\substack{k+\frac{1}{2} \\ 0, i+\frac{1}{2}, j+\frac{1}{2}}} .
$$

To curnlete the method, we update

$$
\Phi_{i+\frac{1}{2}, j+\frac{1}{2}}^{k+1}=\Phi_{i+\frac{1}{2}, j+\frac{1}{2}}^{k+\frac{1}{2}} I_{0, i+\frac{1}{2}, j+\frac{1}{2}}^{k+1} / I_{0, i+\frac{1}{2}, j+\frac{1}{2}}^{k+\frac{1}{2}} .
$$

It is seen that, by construction, Eq. (41) will have the same groupsummed solution as Eq. (44) on convergence. One cannot prove convergence of this procedure; however, there exists a similar linear method 18 which one can prove does converge in model problems. Our experience has shown that the procedure based on Eqs. (41) and (44) does converge for all tested problems in much the same way as the linear methods.

We make the observation that Eq. (44) is a diffusion equation with an unsymmetric operator and that there is no general method for solving it. At present we have some crude methods for solution based on the Gauss-Seidel technique. In the future we plan to investigate a multigrid method which is the current diffusion solver in TWODANT.

Thus our current solution method $\mathrm{f} c \mathrm{r}$ the stationary radiationhydrodynamics transport equation consists of Eqs. (33)-(36), (41), and (44) with the auxiliary Eqs. (37), (40), and (46). We present some preliminary results which include the parameter iteration to convergence for some simple problems to illustrate the current status of implementation.

The test problem is a cylinder composed of a single material whose opacity is described by the analytic expression

$$
\sigma(v, T)=\frac{\sigma_{0}}{(h v)^{3}}\left(1-e^{-h v / k T}\right)
$$

where $\sigma_{0}$ is a constant.

The cylinder has a half-height of $20 \mathrm{~cm}$ and a $20-\mathrm{cm}$ radius with a constant source at $Z=0.0$ for $0.0 \leq R \leq 20 \mathrm{~cm}$ whose temperature is $1 \mathrm{keV}$. The transport and energy balance equations, (26) and (27), with full parameter 
iteration are solved for an infinite time step to obtain the converged, asymptotic temperature distribution and the corresponding intensities. The discretization of the problem is as follows: $S_{4}$ angular quadrature, 10 frequency groups, $70 \times 70$ uniform spatial mesh intervals. The parameter iteration was continued until the maximum pointwise temperature change was less than 0.001 , that is,

$$
\max \left|1-\mathrm{T}_{i, j}^{\mathrm{p}+1} / \mathrm{T}_{i, j}^{\mathrm{p}}\right| \leq 0.001
$$

where $\mathrm{p}$ is the parameter iteration index. The solution was achieved with 10 parameter iterations, 10 transport solutions of Eq. (33), 44 diffusion iterations on $\mathrm{Eq}$. (41), and $53 \mathrm{cpu}$ seconds of CRAY computer time. We also tested the effectiveness of the iteration acceleration procedures as described in this report. We established the convergence rates by holding the temperature constant (no parameter iteration) and letting the iterations proceed. The results are that the multigroup synthetic diffusion acceleration of the transport iterations has a spectral radius of 0.23 while the one-group acceleration of the multigroup diffusion equation has a spectral radius of 0.76 . These compare well with the analytical and experimental results reported in Ref. 18 . In a future report we will expand upon these results and extend them to the time-dependent case.

D. Acceleration of High-Order $S_{N}$ Equations Using Low Order $S_{N}$ Operators (E. Larsen)

The problem of accelerating the convergence of $S_{N}$ calculations is an old one which has receiveo much attention in recent years due to the emergence of stable and effective diffusion synthetic acceleration (DSA) methods, 21, 23, 24 These methods have been proved efficient for numerous spatial differencing schemes in slab Beometry, and for the diamond-difference scheme in all of the commonly-used transport geometries. However, because of algebraic difficulties, the method has not been surcessfully implemented for non-diamonddifferencing schemes in non-slab geometries. Thus, there is a strong incentive to consider other ways to accelerate $S_{N}$ calculations, wi th the goal of deriving more flexible methods which can be used in problems for which standard DSA methods cannot be used. 
In this report we discuss several new iterative methods which potentially can overcome the difficulties with the DSA method discussed above. These methods characterize themselves by using low order $S_{N}$ operators $(N=2$ or 4$)$ to accelerate the convergence of high-order $S_{\mathrm{N}}$ equations, as opposed to the use of the diffusion operator which is employed in DSA methods. In this preliminary study, we consider specifically the acceleration of the exact $\left(S_{\infty}\right)$ transport equation in infinite media using $S_{2}$ and $S_{4}$ operators, and we do not consider spatial discretization methods. The remainder of this report consists of two parts: in part 1 we discuss slab geometry, and in part 2 we discuss $(x, y)$ geometry.

1. Slab Geometry. We wish to solve the equation

$$
\mu \frac{\partial}{\partial x} \psi(x, \mu)+\psi(x, \mu)=\frac{c}{2} \int_{-1}^{1} \psi\left(x, \mu^{\prime}\right) d \mu^{\prime}+Q(x)
$$

The standard iterative method for this equation can be written

$$
\begin{aligned}
& \mu \frac{\partial}{\partial x} \psi^{\ell+\frac{1}{2}}+\psi^{\ell+\frac{1}{2}}=c \phi^{l}(x)+Q, \\
& \phi^{\ell+\frac{1}{2}}(x) \equiv \frac{1}{2} \int_{-1}^{1} \psi^{\ell+\frac{1}{2}}\left(x, \mu^{\prime}\right) d \mu^{\prime}, \\
& \phi^{\ell+1}(x)=\phi^{\ell+\frac{1}{2}}(x) .
\end{aligned}
$$

We shall modify this method by replacing Eq. (49c) with an $S_{2}$ equation which produces the exact result in one iteration if the transport operator in $E q$. $(49 c)$ is replaced by the $S_{2}$ operator. Thus, we define

$$
\psi_{1}(x)=\int_{0}^{1} \psi(x, \mu) d \mu
$$




$$
\begin{aligned}
& \mu_{1}=\frac{1}{\sqrt{3}}, \quad w_{1}=\frac{1}{2}, \\
& \psi_{2}(x)=\int_{-1}^{0} \psi(x, \mu) d \mu, \\
& \mu_{2}=-\frac{1}{\sqrt{3}}, \quad w_{2}=\frac{1}{2} .
\end{aligned}
$$

We now operate on Eq. (49a) by $\int_{0}^{1}(\cdot) \mathrm{d} \mu$ and $\int_{-1}^{0}(\cdot) \mathrm{d} \mu$ and rearrange, obtaining

$$
\begin{aligned}
& \left(\mu_{1} \frac{d}{d x}+1\right) \psi_{1}^{l+\frac{1}{2}}=c \phi^{l}+Q+\frac{d}{d x} \int_{0}^{1}\left(\mu_{1}-\mu\right) \psi^{l+\frac{1}{2}} d \mu, \\
& \left(\mu_{2} \frac{d}{d x}+1\right) \psi_{2}^{l+\frac{1}{2}}=c \phi^{l}+Q+\frac{d}{d x} \int_{-1}^{0}\left(\mu_{2}-\mu\right) \psi^{l+\frac{1}{2}} d \mu .
\end{aligned}
$$

Now we define acceleration equations by

$$
\begin{aligned}
& \left(\mu_{1} \frac{d}{d x}+1\right) \psi_{1}^{l+1}=c \sum_{n=1}^{2} \psi_{n}^{l+1} w_{n}+Q+\frac{d}{d x} \int_{0}^{1}\left(\mu_{1}-\mu\right) \psi^{l+\frac{1}{2}} d \mu, \\
& \left(\mu_{2} \frac{d}{d x}+1\right) \psi_{2}^{l+1}=c \sum_{n=1}^{2} \psi_{n}^{l+1} w_{n}+Q+\frac{d}{d x} \int_{-1}^{0}\left(\mu_{2}-\mu\right) \psi^{l+\frac{1}{2}} d \mu .
\end{aligned}
$$

Subtracting Eqs. (50) from Eqs. (51), we get 


$$
\begin{gathered}
\left(\mu_{m} \frac{d}{d x}+1\right)\left(\psi_{m}^{l+1}-\psi_{m}^{l+\frac{1}{2}}\right)-c \sum_{n=1}^{2}\left(\psi_{n}^{l+1}-\psi_{n}^{l+\frac{1}{2}}\right) w_{n} \\
=c\left(\phi^{l+\frac{1}{2}}-\phi^{\ell}\right), \quad m=1,2 .
\end{gathered}
$$

Thus, the proposed iteration method is

$$
\begin{aligned}
& \left(\mu \frac{\partial}{\partial x}+1\right) \psi^{l+\frac{1}{2}}=c \phi^{l}+Q, \\
& \psi_{1}^{\ell+\frac{1}{2}}=\int_{0}^{1} \psi^{\ell+\frac{1}{2}} d \mu,
\end{aligned}
$$

$\psi_{2}^{\ell+\frac{1}{2}}=\int_{-1}^{0} \psi^{\ell+\frac{1}{2}} \mathrm{~d} \mu$

$$
\phi^{\ell+\frac{1}{2}}=\sum_{n=1}^{2} \psi_{n}^{\ell+\frac{1}{2}} w_{n}
$$$$
\left(\mu_{m} \frac{d}{d x}+1\right)\left(\psi_{m}^{l+1}-\psi_{m}^{l+\frac{1}{2}}\right)-c \sum_{n=1}^{2}\left(\psi_{n}^{l+1}-\psi_{n}^{l+\frac{1}{2}}\right) w_{n}=c\left(\phi^{l+\frac{1}{2}}-\phi^{l}\right) \text {, }
$$

$$
\phi^{l+1}=\sum_{n=1}^{2} \psi_{n}^{\ell+1} w_{n}
$$

The Fourier analysis for this method is performed in the usual way; thus, for $-\infty<\lambda<\infty$ we introduce 


$$
\begin{aligned}
& Q=0, \\
& \phi^{\ell}=\omega^{\ell} e^{i \lambda x}, \\
& \psi^{\ell+\frac{1}{2}}=\omega^{\ell} a(\mu) e^{i \lambda x}, \\
& \psi_{j}^{\ell+\frac{1}{2}}=\omega^{\ell} a_{j} e^{i \lambda x}, \\
& \phi^{\ell+\frac{1}{2}}=\omega^{\ell} A e^{i \lambda x}, \\
& \psi_{m}^{\ell+1}=\omega^{\ell} u_{m} e^{i \lambda x},
\end{aligned}
$$

into Eqs. (52) and solve for tiic eigenvalue $\omega$, obtaining

$$
\omega=\frac{\Lambda_{\infty}-\Lambda_{2}}{1-\Lambda_{2}},
$$

where

$$
\Lambda_{\infty}=c \int_{0}^{1} \frac{d \mu}{1+\lambda^{2} \mu^{2}}
$$

and

$$
\Lambda_{2}=\frac{\mathrm{c}}{1+\lambda^{2} / 3}
$$

For every $0 \leq c \leq 1$, the denominator in Eq. (53) is minimized by setting $c=1$. Doing this, and then numerically computing the maximum over $\lambda$ of the remaining expression, we find that the spectral radius $\rho$ of the iteration method (5a) satisfies

$$
\rho=\sup _{\lambda}|\omega| \leq(0.224) \subset
$$


Hence, the iteration method (52) converges with a rate equal to that for the standard DSA method.23,24 This result is not totally unexpected, because the $S_{2}$ equations in slai geometry are known to be equivalent to the diffusion equation, which is the basis for the DSA method. However, discretized versions of Eqs. (52) are not in general equivalent to discretized versions of the DSA equations.

2. $(x, y)$ Geometry. Nuw we wish to solve the equation

$$
\underline{\Omega} \cdot \underline{\nabla} \psi(\underline{x}, \underline{\Omega})+\psi(\underline{x}, \underline{\Omega})=\frac{\mathrm{c}}{4 \pi} \int \psi\left(\underline{x}, \underline{\Omega}^{\prime}\right) d \Omega^{\prime}+Q(\underline{x}),
$$

where $\underline{x}=(x, y, 0)$ and $\left.\right|_{\underline{\Omega}} \mid=1$. An $S_{N}$ quadrature set consists of a set of unit 3-dimensional vectors $\Omega_{m}$ and weights $w_{m}$, and associated with each $\Omega_{m}$ is a "cone" $C_{m}$ on the unit sphere with area $4 \pi w_{n}$ :

$$
\begin{aligned}
& \int_{C_{m}} d \Omega=4 \pi w_{m}, \\
& \sum_{n=1}^{N} w_{n}=1 .
\end{aligned}
$$

We define

$$
\psi_{m}(\underline{x})=\frac{1}{4 \pi w_{m}} \int_{C_{m}} \psi(\underline{x}, \underline{\Omega}) d \Omega .
$$

The iterative method in $x, y$ - geometry which is analogous to that of Eq. (52), and which can be derived in a similar fashion, is:

$$
(\underline{\Omega} \cdot \underline{\nabla}+1) \psi^{\ell+\frac{1}{2}}(\underline{x}, \underline{\Omega})=c \phi^{l}(\underline{x})+Q,
$$




$$
\begin{aligned}
& \psi_{m}^{l+\frac{1}{2}}(\underline{x})=\frac{1}{4 \pi w_{m}} \int_{C_{m}} \psi^{l+\frac{1}{2}}(\underline{x}, \underline{\Omega}) d \Omega, m=1, \ldots, N \\
& \phi^{\ell+\frac{1}{2}}(\underline{x})=\sum_{n=1}^{N} \psi_{n}^{\ell+\frac{1}{2}}(\underline{x}) w_{n}, \\
& \left(\underline{\Omega}_{m} \underline{\nabla}+1\right)\left(\psi_{m}^{l+1}-\psi_{m}^{l+\frac{1}{2}}\right)-c \sum_{n=1}^{N}\left(\psi_{n}^{l+1}-\psi_{n}^{l+\frac{1}{2}}\right)_{n} \\
& =c\left(\phi^{l+\frac{1}{2}}-\phi^{l}\right),\left(\underline{\Omega}_{m} \in S_{N}\right) \\
& \phi^{l+1}=\sum_{n=1}^{N} \psi_{n}^{l+1} w_{n} .
\end{aligned}
$$

The Fourier analysis for this method proceeds exactly as in the slab geometry case, and we find

$$
\omega=\frac{\Lambda_{\infty}-\Lambda_{N}}{1-\Lambda_{N}},
$$

where

$$
\Lambda_{\infty}=c \int_{0}^{1} \frac{d \mu}{1+\lambda^{2} \mu^{2}}
$$

and

$$
\Lambda_{N}=c \sum_{n=1}^{N} \frac{w_{n}}{1+i \underline{\lambda} \cdot \underline{\Omega}_{n}} \text {, }
$$


and $\underline{\lambda}$ is a three-dimensional vector lying in the $(x, y)$ plane with components

$$
\underline{\lambda}=(\lambda \cos \theta, \lambda \sin \theta, 0) .
$$

We have

$$
\lim _{\lambda \rightarrow \infty} \Lambda_{\infty}=0
$$

and

$$
\lim _{\lambda \rightarrow \infty} \sup \Lambda_{N}=2 c\left[\max _{1 \leq n \leq N} w_{n}\right] \equiv 2 c w_{\max }
$$

Thus, Eq. (56) gives

$$
\lim \sup _{\lambda \rightarrow \infty}|\omega|=\frac{2 c w_{\max }}{1-2 c w_{\max }} .
$$

For the $S_{2}$ quadrature set, all $w_{n}=\frac{1}{4}$ and for the $S_{4}$ quadrature set, all $w_{n}=1 / 12$. Experimentally, we find that the largest values of $|\omega|$ occur at $|\lambda|=\infty$; thus, the spectral radil for these quadrature sets are

$$
\begin{aligned}
& \rho_{2}=\frac{c}{2-c}, \\
& \rho_{4}=\frac{c}{6-c} .
\end{aligned}
$$

Thus as $c \rightarrow 1, p_{2} \rightarrow 1$ and $p_{4} \rightarrow 0.2$. Hence, the above $S_{2}$ acceleration method is stable but becomes ineffective, while the $S_{4}$ method remains stable and effective. The result (59) is identical to a result obtained by Gelbard and Hageman 25 for a slightly different acceleration method. The curves

$$
\omega^{*}(\lambda)=\sup _{0 \leq \theta \leq 2 \pi}|\omega(\lambda, \theta)|
$$


for the method of Eqs. (55), with $c=1$ and $N=2$ and 4 , are displayed in Fig. 4 .

In the following, we discuss three ways to modify the iteration method (55) so as to make the method effective when used with $\mathrm{N}=2$.

The first such method is derived by noting that with $c=1$ and $N=2$, the curve $\omega^{*}$ versus $\lambda$ for the iteration method (55) monotonically increases from 0 to 1 as $\lambda$ increases from o to $\infty$ [see Fig. 4], while the corresponding curve for the unaccelerated transport sweep [defined by Eq. (57)] monotonically decreases from 1 to 0 . Thus, if we include in the iteration method (55) one extra transport sweep, the eigenvalue of the resulting method will be bounded from above by the product of the above two eigenvalues, and hence will be bounded less than one. This method is explicitly defined by

$$
\begin{aligned}
& (\underline{\Omega} \cdot \underline{\nabla}+1) \psi^{\ell+1 / 3}(\underline{x}, \underline{\Omega})=c \phi^{l}(\underline{x})+Q(\underline{x}) \\
& (\underline{\Omega} \cdot \underline{\nabla}+1) \psi^{\ell+2 / 3}(\underline{x}, \underline{\Omega})=c \phi^{l+1 / 3}(\underline{x})+Q(\underline{x}) \\
& \left(\underline{\Omega}_{m} \cdot \underline{\nabla}+1\right)\left(\psi_{m}^{l+1}-\psi_{m}^{l+2 / 3}\right)-c \sum_{n=1}^{4}\left(\psi_{n}^{l+1}-\psi_{n}^{l+2 / 3}\right) w_{n} \\
& =c\left(\phi^{l+2 / 3}-\phi^{l+1 / 3}\right),\left(\underline{\Omega}_{m} \in S_{2}\right)
\end{aligned}
$$

where the definitions of $\phi^{\ell+1 / 3}, \phi^{\ell+2 / 3}, \psi_{m}^{\ell+2 / 3}$, and $\phi^{\ell+1}$ should be obvious from the discussion above. The Fourier analysis for this method leads to the result

$$
\omega=\Lambda_{\infty} \frac{\Lambda_{\infty}-\Lambda_{2}}{1-\Lambda_{2}},
$$

and numerically we find

$$
\rho \leq(0.152) \mathrm{C}
$$




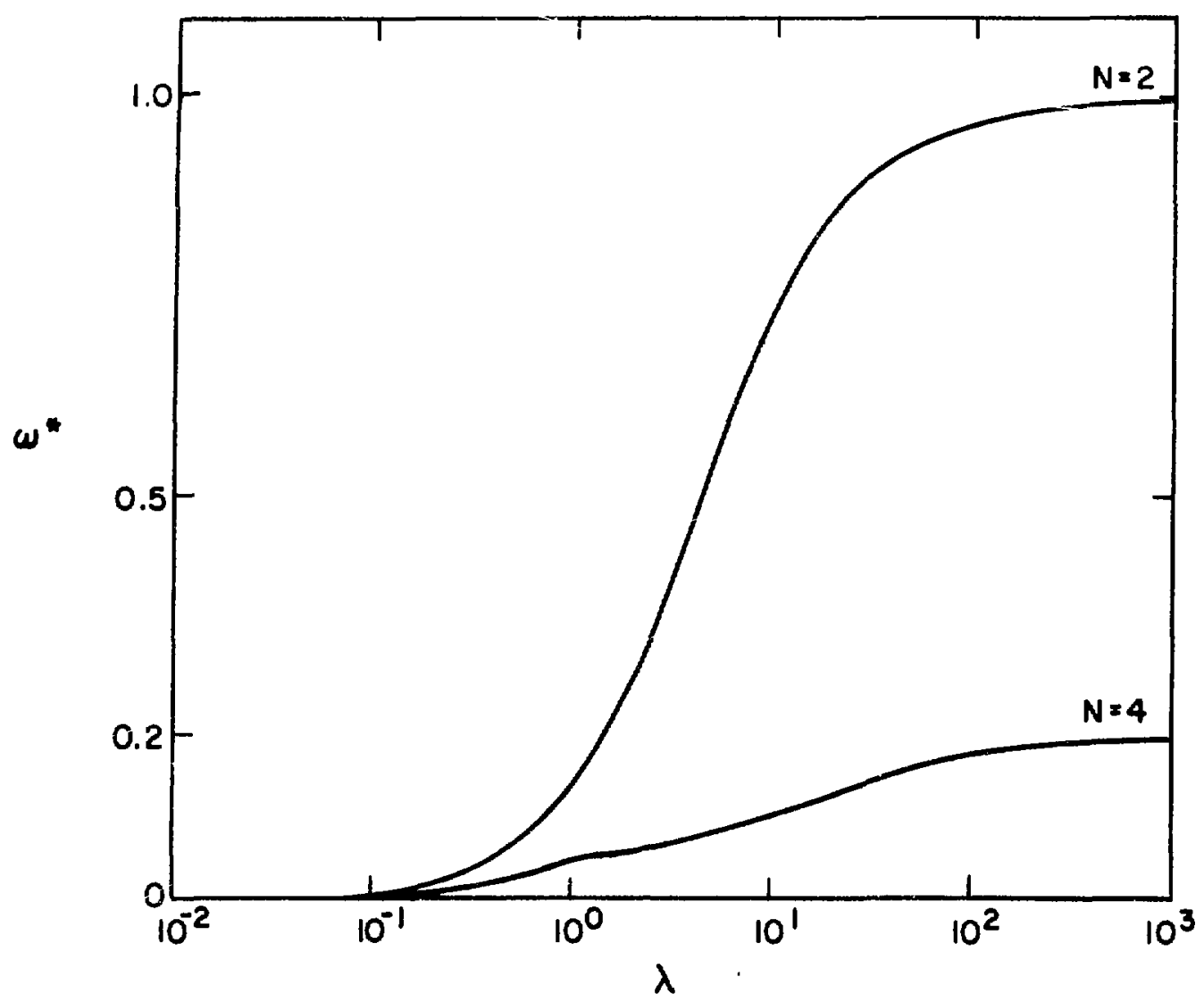

Fig. 4. $\omega^{*}$ vs $\lambda$ for method (55), with $c=1$ and $N=2$ and 4 . 
Thus, this method is stable and effective. The curves $\omega^{*}$ versus $\lambda$ for the methods of Eqs. (60), (55) and the unaccelerated transport sweep are displayed in Fig. 5 .

The second and third methods are based on replacing one of the two transport sweeps in tile method $(60)$ by an $S_{4}$ sweep. Conceptually, either Eq. (60a) or (60b) can be replaced, and thus we obtain two distinct methods. These ran be written

$$
\begin{aligned}
& (\underline{\Omega} \cdot \underline{\nabla}+1) \psi^{l+1 / 3}=c \phi^{l}+Q, \\
& \left(\underline{\Omega}_{k} \cdot \underline{\nabla}+1\right)\left(\psi_{k}^{l+2 / 3}-\psi_{k}^{l+1 / 3}\right)=c\left(\phi^{l+1 / 3}-\phi^{l}\right),\left(\underline{\Omega}_{k} \in S_{4}\right) \\
& \left(\underline{\Omega}_{m} \underline{\nabla}+1\right)\left(\psi_{m}^{l+1}-\psi_{m}^{l+2 / 3}\right)-c \sum_{n=1}^{4}\left(\psi_{n}^{l+1}-\psi_{n}^{l+2 / 3}\right) w_{n} \\
& =c\left(\phi^{l+2 / 3}-\phi^{l+1 / 3}\right),\left(\underline{\Omega}_{m} \in S_{2}\right),
\end{aligned}
$$

and

$$
\begin{aligned}
& \underline{(\Omega} \cdot \underline{\nabla}+1) \psi^{\ell+1 / 3}=c \phi^{\ell}+Q \text {, } \\
& \left(\underline{\Omega}_{m} \cdot \underline{\nabla}+1\right)\left(\psi_{m}^{\ell+2 / 3}-\psi_{m}^{\ell+1 / 3}\right)-c \sum_{n=1}^{4}\left(\psi_{n}^{\ell+2 / 3}-\psi_{n}^{\ell+1 / 3}\right) w_{n} \\
& =c\left(\phi^{l+1 / 3}-\phi^{\ell}\right), \quad\left(\Omega_{m} \in S_{\varepsilon}\right) \text {, } \\
& \left(\underline{\Omega}_{k} \cdot \underline{\nabla}+1\right)\left(\psi_{k}^{l+1}-\psi_{k}^{l+1 / 3}\right)=c\left(\phi^{\ell+2 / 3}-\phi^{l}\right),\left(\underline{\Omega}_{k} \varepsilon S_{4}\right),
\end{aligned}
$$

Where, again, the definitions of the various quantities should be clear from the discussions above. The Fourier analyses for these methods lead to ident1cal results, namely 


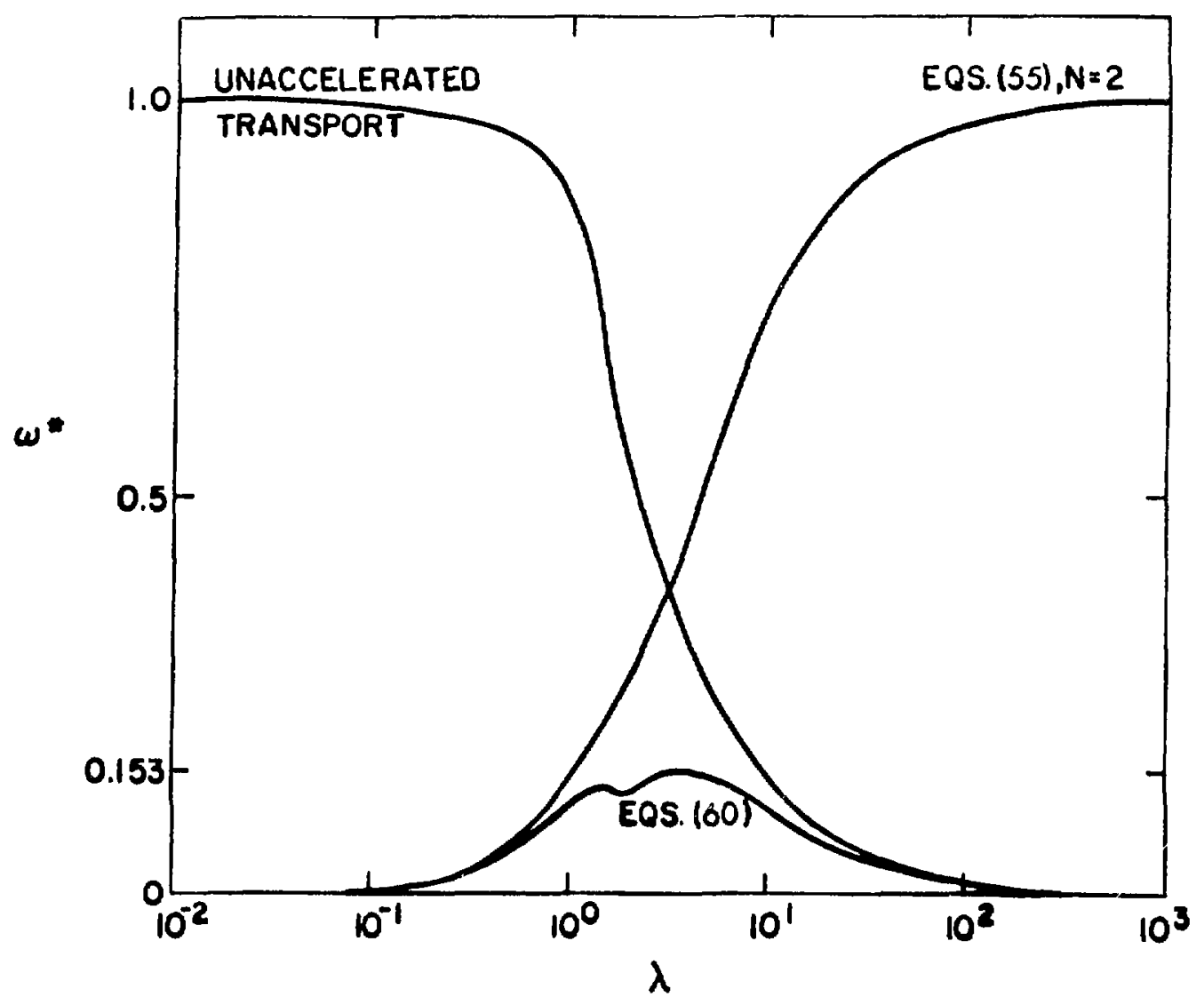

Fig. 5. $\omega^{*}$ vs $\lambda$ for methods $(60),(55)$ with $N=2$, and the unaccelerated transport sweep $(c=1)$. 
Thus, this method is stable and effective. The curves $\omega^{*}$ versus $\lambda$ for the methods of Eqs. (60), (55) and the unaccelerated transport sweep are displayed in Fig. 5 .

The second and third methods are based on replacing one of the two transport sweeps in the method $(60)$ by an $S_{4}$ sweep. Conceptually, either Eq. $(60 \mathrm{a})$ or $(60 \mathrm{~b})$ can be replaced, and thus we obtain two distinct methods. These can be written

$$
\begin{aligned}
& \underline{(\Omega} \cdot \underline{\nabla}+1) \psi^{l+1 / 3}=c \phi^{l}+Q, \\
& \left(\underline{\Omega}_{k} \cdot \underline{\nabla}+1\right)\left(\psi_{k}^{l+2 / 3}-\psi_{k}^{l+1 / 3}\right)=c\left(\phi^{\ell+1 / 3}-\phi^{l}\right),\left(\underline{\Omega}_{k} \varepsilon S_{4}\right) \\
& \left(\underline{\Omega}_{m} \cdot \underline{\nabla}+1\right)\left(\psi_{m}^{l+1}-\psi_{m}^{l+2 / 3}\right)-c \sum_{n=1}^{4}\left(\psi_{n}^{l+1}-\psi_{n}^{l+2 / 3}\right) w_{n} \\
& =c\left(\phi^{l+2 / 3}-\phi^{l+1 / 3}\right),\left(\underline{\Omega}_{m} \varepsilon S_{2}\right),
\end{aligned}
$$

and

$$
\begin{aligned}
& (\underline{\Omega} \cdot \underline{\nabla}+1) \psi^{\ell+1 / 3}=c \phi^{l}+Q, \\
& \left(\underline{\Omega}_{m} \cdot \underline{\nabla}+1\right)\left(\psi_{m}^{\ell+2 / 3}-\psi_{m}^{l+1 / 3}\right)-c \sum_{n=1}^{4}\left(\psi_{n}^{\ell+2 / 3}-\psi_{n}^{\ell+1 / 3}\right) w_{n} \\
& =c\left(\phi^{\ell+1 / 3}-\phi^{l}\right), \quad\left(\underline{\Omega}_{m} \varepsilon S_{2}\right), \\
& \left(\underline{\Omega}_{k} \cdot \underline{\nabla}+1\right)\left(\psi_{k}^{\ell+1}-\psi_{k}^{\ell+1 / 3}\right)=c\left(\phi^{\ell+2 / 3}-\phi^{l}\right),\left(\underline{\Omega}_{k} \varepsilon S_{4}\right),
\end{aligned}
$$

where, again, the definitions of the various quantities should be clear from the discussions above. The Fourier analyses for these methods lead to identical results, namely 


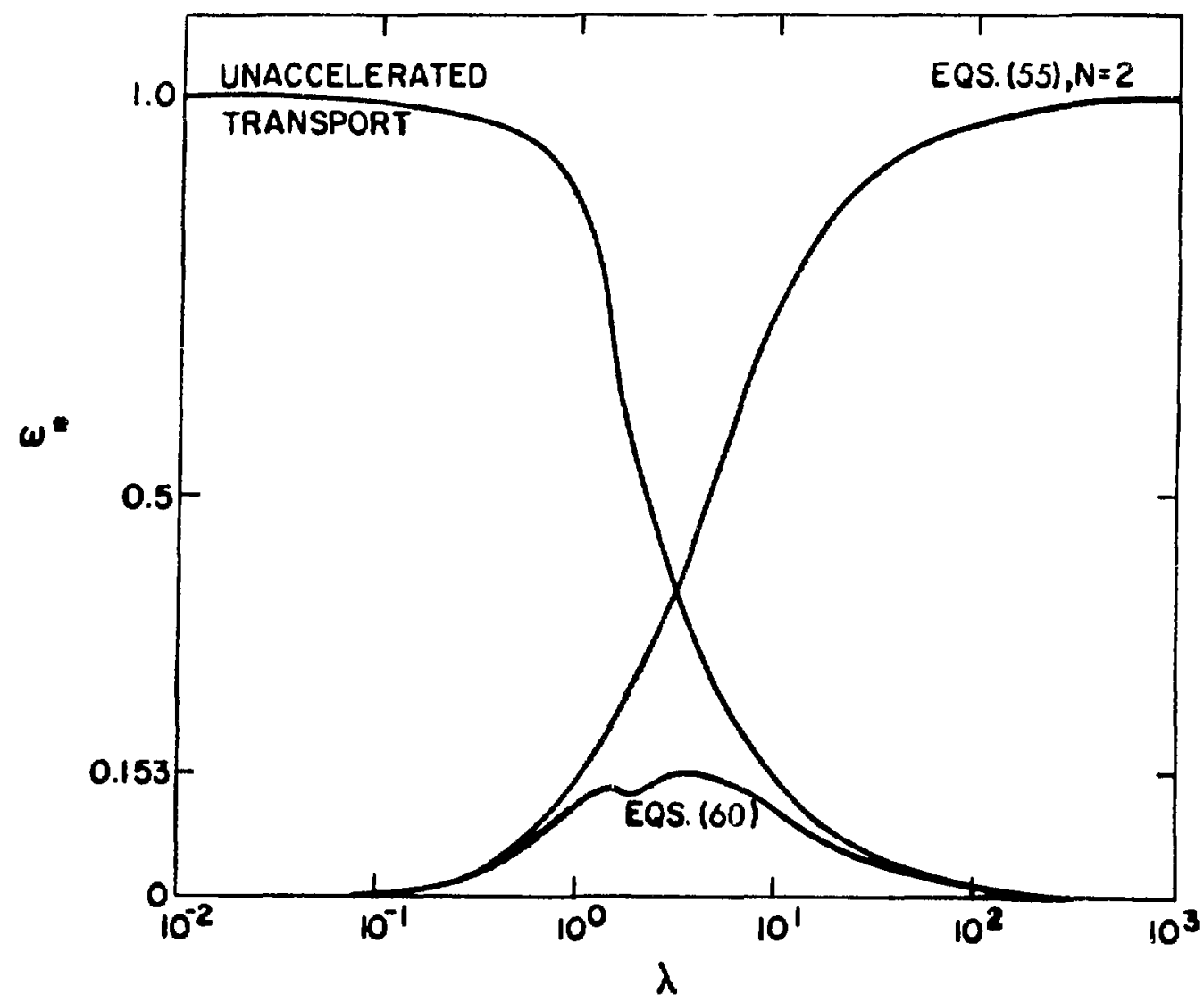

Fig. 5. $\omega^{*}$ vs $\lambda$ for methods $(60),(55)$ with $N=2$, and the unaccelerated transport sweep $(c=1)$. 


$$
\omega=\frac{\Lambda_{\infty}-\Lambda_{4}+\Lambda_{\infty}\left(\Lambda_{4}-\Lambda_{2}\right)}{1-\Lambda_{2}},
$$

and numerically we 'f ind

$$
\rho \leq \frac{c}{3}
$$

Thus, these methods also are stable and effective. The curve $\omega^{*}$ versus $\lambda$ for the methods (62) and (63) is displayed in Fig. 6.

To summarize, we have described four methods for effectively accelerating the convergence of $x, y$-geometry transport iterations using $S_{2}$ and $S_{4}$ operators:

\begin{tabular}{|l|l|l|}
\hline $\begin{array}{l}\text { Description of } \\
\text { One Iteration }\end{array}$ & $\begin{array}{l}\text { Equation } \\
\text { \# }\end{array}$ & $\begin{array}{l}\text { Spectral } \\
\text { Radius }\end{array}$ \\
\hline $\begin{array}{l}1 \text { Transport sweep } \\
1 \text { full S4 calculation }\end{array}$ & $(55)$ & $(0.2) c$ \\
\hline $\begin{array}{l}\text { Transport sweeps } \\
1 \text { full S calculation }\end{array}$ & $(60)$ & $(0.153)^{c}$ \\
\hline 1 Transport sweep \\
1 S sweep \\
1 full S calculation
\end{tabular}

To provide a solid theoretical basis for these methods, it remains to perform a Fourier analysis for the spatially discretized equations and again demonstrate stability and effectiveness. In addition, a procedure for efficiently solving the full $\mathrm{S}_{2}$ and $\mathrm{S}_{4}$ equations must be devised. These will be the subject of future study.

E. TRIDENT-CTR and the Streaming Matrix Hybrid Method (Bradley A. Clark)

The Streaming Matrix Hybrid Method (SMHM) 26 has been fully incorporated into the TRIOENT-CTR 27 code. During this reporting period, the SMHM was tested on two realistic duct streaming problems; comparisons were made with 


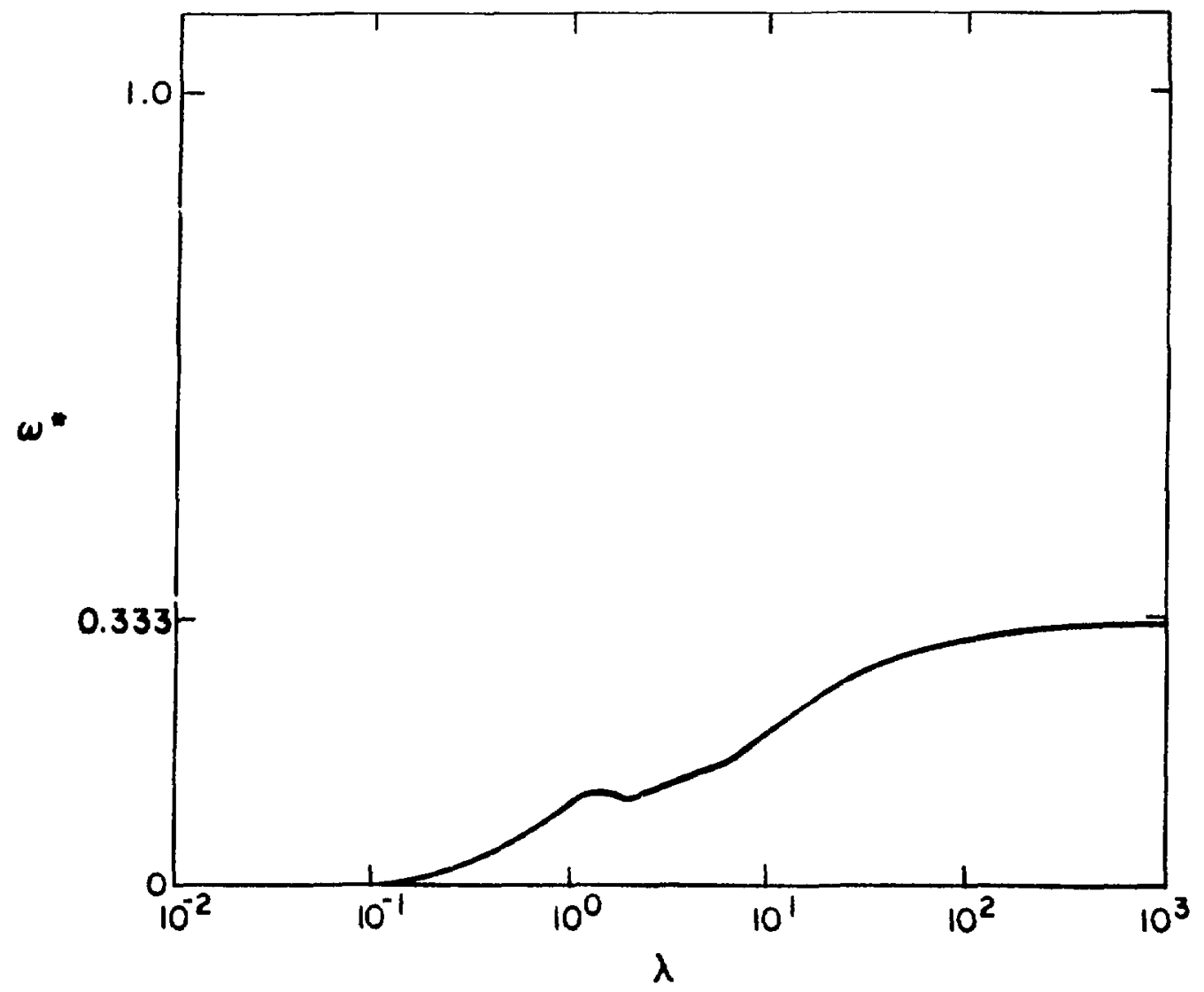

Fig. 6. $\omega^{*}$ vs $\lambda$ for methods $(62)$ and $(63)(c=1)$. 
standard discrete-ordinates and Monte Carlo calculations. The results of the study are reported in detail elsewhere. 28 In summary, the SMHM continues to show promise as an alternative to discrete-ordinates. Although the SMHM does not completely eliminate ray-effects, it significantly reduces them and calculates accurate fluxes and currents in material adjacent to voids.

Following the aforementioned study, the responsibili y for TRIDENT-CTR, including the SMHM version, and its post-processors, was transferred to Donald J. Dudziak of Group S-4 at Los Alamos National Laboratory. Any future questions concerning TRIDENT-CTR should be sent to its new caretaker.

IV. MONTE CARLO RADIATION TRANSPORT

Group $X-6$ devotes a significant effort to the development, implementation, assessment, and application of Monte Carlo methods and codes for radiation transport calculations.

For this reporting period, we report on an MCNP User's Workshop held for users in the Magnetic Fusion Energy (MFE) community. This is followed by a report on work on our main Monte Carlo code, MCNP, with contributions from several of our staff. Next is a report on a perturbation code version of MCNP and on Monte Carlo learning/biasing techniques. In other Monte Carlo code development and applications areas, we present a report on our progress on the CYLTRAN code for coupled electron/photon transport. This is followed by a report on Monte Carlo simulation of electron/photon/Cerenkov cascades in general geometry using the ACCEPT Monte Carlo code. We next describe our work on the CONPAR graphics code for displaying the geometry enviscnments to be used in Monte Carlo calculations. The final report in this section describes comparative calculations on a light watei reactor shielding benchmark using continuous-energy Monte Carlo, multigroup Monte Carlo, and multigroup discrete-ordinates codes.

A. MCNP Workshop

On October 4-7, 1983, a workshop on MCNP was given to the Magnetic Fusion Energy (MFE) users of MCNP. MCNP is now used extensively throughout the MFE community as their main Monte Carlo neutronics code. 
The four-day workshop explained the practical aspects of MCNP. Particular attention was given to the tallies, variance reduction techniques, and the output from a user-oriented point of view. For a fairly difficult problem, essentially every variance reduction technique in MCNP was tried to illustrate its effect.

The topics covered in the workshop included an introduction to Monte Carlo, the geometry, physics, cross section, sources, tallies, variance reduction, input, output, coding, several major patches (multigroup, adjoint, surface sources, importance generator, perturbations, exponential transform), and future plans.

The entire workshop was videotaped, producing a total of 27 tapes. A set of the tapes is available from the $X-6$ Group office, the MFE library at Livermore, and the Radiation Shielding Information Center at Oak Ridge.

Outside organizations can purchase their own set of tapes. Contact Jim Hudgins, IS-2, MS-D415, Los Alamos National Laboratory. Abstracts of each of the topics are also available as are hard copies of all the viewgraphs used. B. MCNP Maintenance, Improvement, and Support

On September 28, 1983, Version 3 of our general purpose Monte Carlo code MCNP14 was put into production at Los Alamos and was sent to the Radiation Shielding Information Center (RSIC) at Oak Ridge for distribution to users in the radiation shielding community.

During this reporting period, several members of Group $x-6$ have devoted effort to the maintenance, improvement, and support of MCNP. Their individual contributions are described below.

1. Version $3 A$ of MCNP. (T. N. K. Godfrey) - I have been working on several features to be included in Version $3 \mathrm{~A}$ of MCNP, which we plan to issue sometime in 1984 .

The most important change in Version $3 \mathrm{~A}$ will be a complete overhaul of the sections of MCNP that set up the source and sample the variables of each source particle. Our objective is to provide source facilities that are so general and flexible that the need for users to provide source capabilities by means of modifications to MCNP itself will be greatly reduced. At present it is quite common for a user to provide his own source subroutines. We hope that in the future it will be rare. 
My starting point is a generalized source patch that Bob Sohrandt has been working on and Guy Estes has been using in his applications. Most of the new concepts and new input language for the Version $3 \mathrm{~A}$ source are included in Schrandt's patch. I am working on integrating the patch into MCNP and, together with Schrandt, making it even more general.

I have been working on a repeated structures capability for MCNP. It is described in detail in my memo, "Repeated Structures in MCNP".29 The objective is to reduce the amount of input data the user has to providt und the amount of computer memory needed by problems that have a lot of geometrical repetition. The repetition pattern may be a lattice or just a set of similar objects. So far I have a correctly running patch that incorporates most of the repeated structures features. A few features have yet to be done, notably a hexagonal prism lattice and a scheme for handling small exceptions in the repetition.

I have written an improved FM card package that allows a $b$ in to have both an attenuator and a response function. Those two features are presently incompatible.

I have written a patch which will be included in Version $3 \mathrm{~A}$ which will allow MCNP input data to be in columns rather than just rows. It is described in my memo, "Column Input for MCNP".30 It would, in some cases, make the input much more readable and make it less likely that a user would make errors when modify ing an input file.

2. Qualification of the MCNP Code. (R. E. Seamon) - The MCNP Monte Carlo code is used throughout the Laboratory on a host of projects including many carried out under the auspices of outside contractors. The question was raised by one such contractor about the "certification document" for MCNP. No such document exists nor do we expect to produce such a document. However, in Ref. 31 we summarized the evidence we have for being confident that MCNP is doing its job well.

3. Work on Version 3, MCNP. (R. C. Little and R. E. Seamon) - One of the reasons given for the massive re-write of MCNP in Fortran 77 is that the code should be exportable and readily compiled on a host of computing machines and operating systens. Associated with this code exportability, it has been deemed necessary to introduce two additional formats for the Monte Carlo cross sections so that now they exist in each of three modes: 
Mode $1-B C D$

Mode 2 - Binary

Mode 3 - ACE format (that format used exclusively before version 3).

Along with the cross-section files, there must now also be cross-section directories. The difficulties and possibilities for error are only now at the time of this writing (February 1984) beginning to be understood. We have already gained some experience in these additional complexities. When special cross-section files are prepared for users, special cross-section directories must be prepared as well.

In connection with Version 3, a new version of MCPLIBID was prepared in which there are atomic weight ratios for all elements as they occur in nature as well as for all the stable isotopes and all other isotopes for which there are cross sections on the dosimetry libraries. That work is described in Ref. 32 .

Prior to the release of Version 3, we ran a host of problems to check the operation of the FM cards similar to the Version $2 C$ testing which we reported in Ref. 33. A series of runs was made on simple problems and hand calculations were carried out to verify the results. Several bugs were uncovered and changes in the coding were suggested to correct the situations. It should be emphasized that this work was done using Mode 3 cross sections only.

4. Cross Sections in our Exportable Version of MCNP. (R. E. Seamon) The exportable MCNP code package must include cross sections, of course; without them it is impossible to make calculations with MCNP. Because of restrictions imposed by the Department of Energy on the distribution of ENDF/B-V data outside of the United States, it has been necessary to provide older crosssection sets to the Radiation Shielding Information Center through which organization the distribution of MCNP is being handled.

5. MCNP Activities. (J. F. Briesmeister) - Version 3 of MCNP activities included writing a flow diagram of the code for the new manual, converting cross-section files and working with Tom Godfrey to understand what the general procedure should be, and writing various tapes. A talk on geometry setup was prepared after the MFE workshop and videotaped to add to the other tapes. 
A patch was written for MCNP to add default file storage and printing capability similar to another $x-6$ code. Plans are to expand its scope and to include the patch in the MCNP family of files.

I have had considerable interaction with the MFE community of users and some contact with RSIC in the past few months.

6. Generalization of MCNP Standard Sources. (R. G. Schrandt and G. P. Estes) - An extensive modification to the standard sources has been turned over to Tom Godfrey for inclusion in Version $3 \mathrm{~A}$ of MCNP.

A "Users Rough Draft" is in preparation. This has been delayed somewhat as changes were made in the philosophy and format of the source definition card.

Hopefully, there will be a reasonable period of time for a friendly users version of this patch, primarily for $x-6$ users. After this period a more definitive report will be issued. This report will include descriptive material on these sources for the new MCNP manual.

A paper was presented on these sources 34 as well as a presentation at the October 1983 MFE Users Workshop.

7. MCNP Patches. (J.S. Hendricks) - I continue to maintain three major MCNP patches. These specially modified versions of MCNP provide capabilities which may eventually be incorporated into the public code. In 1983 these patches were all rewritten to conform to MCNP Version 3.

a. Weight Window Generator Patch. This patch allows the user to specify a tally for which he desires maximum calculational efficiency. The code then computes the optimum importance function using Tom Booth's "forward adjoint" method (References 35,36 ). The weight windows generated by this method are usually--and sometimes spectacularly--better than any importance function which even an experienced user could determine after many trial-anderror attempts.

b. Importance Generator Patch. This patch automatically generates importances by the "on the fly method" described in Refs. 36,37. This method is dynamic: the user inputs no importances and lets the code continuously determine them. The generated importances tend to populate geometry space uniformly rather than optimally.

c. Exponential Transform Patch. This is a generalization of the exponential transform. The transform is no longer limited to y-direction bias; it may now path-length stretch in $x, y, z$, or no direction as well as toward any 
number of arbitrary points in phase space. The stretching parameter may still be a user supplied constant or it may be $p=\Sigma_{a} / \Sigma_{t}$. By setting the transform to the absorption fraction, the distance to scatter rather than distance to collision is sampled. This form of the exponential transform is also equivalent to "absorption weighting Monte Carlo" and "implicit capture along a flight path."

C. Perturbation Code (R. G. Schrandt)

The perturbation patch has been brought up to date to Version 3 of MCNP. This code has had very little use within the laboratory and needs to be tried on a wider variety of problems.

Perturbations of cell density and material are allowed but not of geometry. A void is an acceptabie material perturbation.

One modification to this code allows only a perturbation of cell density and material composition. That is, the same nuclides must occur in both perturbed and nonperturbed materials, but with different possible weight fractions. Correlation is preserved by forcing the perturbed collisions to occur at the site of the unperturbed collision and adjusting the perturbed weight. A single track then represents both the perturbed and unperturbed particles. The particle weights can differ considerably after many collisions or boundary crossings.

This modification has also been brought over to MCNP Version 3 from a patch supplied by Jim Tsang at Schlumberger-Doll. It also allows the perturbed and unperturbed tracks to diverge if the ratio of particular weights exceeds a specified number.

The weight correction resulting from different mean free paths is identical to the exponential transformation corrections in MCNP. A further perturbed weight correction is needed since the probability of picking the scattering nuclide is determined from the unperturbed material composition. This correction is the same as one used in an existing MCNP patch which allows one to bias the scattering from a specified nuclide by introducing a pseudo material card. 
This approach will probably be useful for only a certain type of problem where the perturbation is relatively small and where the nuclei of particle tracks can be reduced considerably by the correlation scheme. Sampling problems can occur when there are large variations in weight associated with the exponential transformation.

D. Angle Bias in MCNP (John S. Hendricks)

Lee Carter (Westinghouse, Hanford) and I have been studying the implementation of angle bias in MCNP.

Angle biasing at collisions can enhance the efficiency of Monte Carlo particle transport calculations by sending particles in a desired direction rather than allowing them to randomly go in undesired directions. Nevertheless, angle biasing is not a widely available feature in modern Monte Carlo particle transport codes for two reasons. First, any modification of the scattering process requires a weight adjustment to keep the Monte Carlo transport game unbiased. This weight modification often introduces unacceptable weight fluctuations. Secondly, modification of the scattering kernel can be very complicated for all but isotropic scattering. This is why Monte Carlo codes that have any form of directional biasing at all usually limit them to isotropic source biasing or to yseudo-deterministic methods such as DXTRAN in MCNP or DXANG as reported by Booth. 38

We have implemented an angular biasing scheme proposed by Dwivedi39 which truly modifies the scattering kernel. Weight fluctuations caused by the angle bias are nearly cancelled by weight fluctuations introduced by the exponential transformation. We call this method the "synergistic" method because the combined angle bias and exponential transform method works far better than either method alone.

As expected, modification of the scattering kernel is complicated. At each collision the scattering kernel must be modified and renormalized by the factor

$$
\int \frac{f(\mu) d \mu}{\left(1-\rho^{2}+\rho^{2} \mu^{2}-2 \rho \mu^{\prime} \mu^{2}+\rho^{2} \mu^{2}\right)^{1 / 2}} \text {. }
$$


For neutron scattering this requires an expensive numerical integration at each collision. Therefore, we have limited our investigation to photons for which $f(\mu)$ can be represented by isotropic or Klein-Nishina scattering formulas. Substituting these formulas into the above normalization integral results in manageable, though very complicated, expressions. Numerous attempts to $f$ ind simpler expressions or to avoid the difficult normalization integration by rejection techniques, splitting methods, etc., were all unsuccessful. We have tested our synergistic method on a number of photon problems. We have discovered a number of difficulties which Dwivedi's one-group onedimensional analysis omitted. We have also found problems for which the synergistic method gains many orders of magnitude improvement in calculational efficiency. In one problem the figure of merit, our. measure of efficiency, went from .11 to 118 . For some problems the use of the synergistic angle bias can eliminate the need to subdivide the geometry into many, many subregions for importance sampling.

A preliminary summary of our work was given at the November, 1983, ANS Meeting 40 and a detailed description is being prepared for submission to Nuclear Science and Engineering.

E. Monte Carlo Learning/Biasing (T. E. Booth)

I continue to make steady progress on Monte Carlo learning/biasing schemes that do their learning/biasing in the random number space rather than the physical phase space. The essential idea is to use biased random numbers rather than a biased transport operator. Briefly, an analog Monte Carlo calculation assigns a random number sequence $\vec{r}$ and a tally $\mathrm{T}(r)$ to each particle history. The calculation then estimates

$$
A=\int T(\vec{r}) P(\vec{r}) d \vec{r}
$$

where $f(\vec{r})$ is a uniform density of random number sequences. Monte Carlo calculations are usually biased by changing $T$ to 'T'. That is to say $\vec{r}$ produces different random walks in the analog and biased cases. To ensure an unbiased estimation, a weight factor, $\omega(r)$, is introduced so that 


$$
\int T^{\prime}(\vec{r}) \omega(\vec{r}) f(\vec{r}) d \vec{r}=\int T(\vec{r}) f(\vec{r}) d \vec{r}
$$

The new idea is to sample $\vec{r}$ from a non-uniform density $g(\vec{r})$ and to account for the bias introduced by adding a weight function $\omega_{g}(\vec{r})$ chosen so that:

$$
\int T(\vec{r}) \omega_{g}(\vec{r}) g(\vec{r}) d \vec{r}=\int T(\vec{r}) f(\vec{r}) d \vec{r}
$$

Using this type of biasing each $\vec{r}$ specifies a particular random walk independent of how much biasing is done. I am working on ideas to have the computer learn what $g(r)$ should be. So far a factor of 50 improvement in the variance has been obtained, al though the time required has gone up by a factor of 80 . I am addressing the time problem now and believe that a solution is in reach. This is the most far-reaching Monte Carlo project $I$ have ever attempted. I have great confidence that the idea is fundamentally sound and that, one way or another, the technique will succeed in a big way.

F. Status of CYLTRAN (H. G. Hughes and J. M. Mack)

CYLTRAN 41 is a general purpose Monte Carlo code for the solution of coupled electron/photon transport problems in cylindrical geometry. The code employs condensed history Monte Carlo for electrons and single scattering Monte Carlo for photons, and covers energies from $100 \mathrm{MeV}$ down to $1 \mathrm{keV}$ for both species. CYLTRAN is part of a family of codes, the Integrated Tiger Series (ITS), which originates at Sandia National Laboratories. We have traditionally maintained a version of CYLTRAN, and friendly-user versions of some of the other codes for Los Alamos users. For continuity with existing applications, and because the special Los Alamos modifications are not yet in the ITS, we still maintain our version of CYLTRAN. During this reporting period we also added a new radially resolved leakage tally and a generallzed conical source.

With the new tally the radial dependences of number, energy, and energy spectra of electrons and photons crossing the transmission or reflection surface are scored. Figure 7 shows the new tally geometry superinposed on a transmission (or reflection) surface of a problem cylinder. Specifically the code now selects a number of concentric rings (annuli) centered on the axis of 


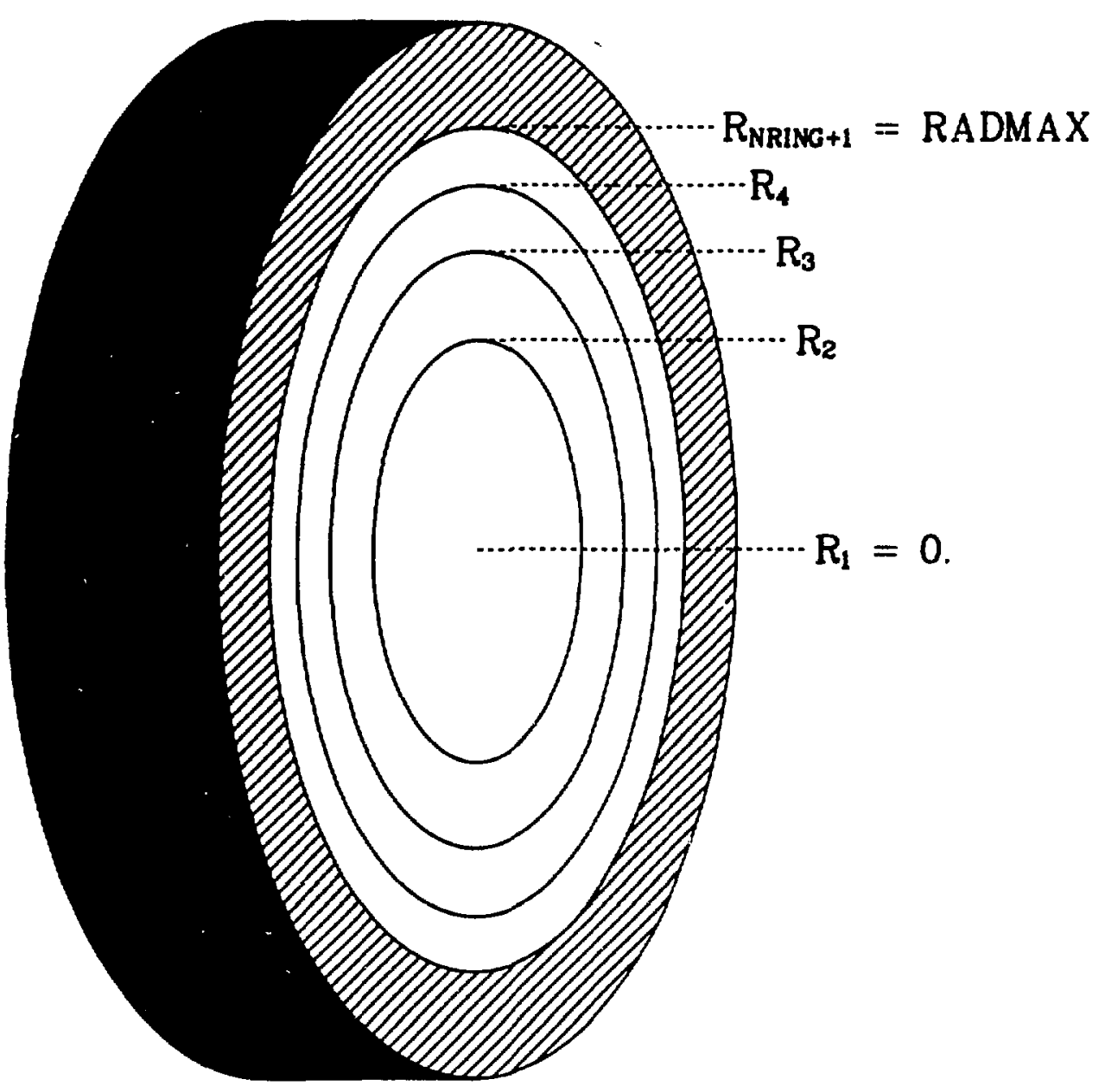

Fig. 7. Radial tally geometry superimposed on a leakage surface of the problem cylinder, illustrated for NRING $=4$. The $i$ th ring is bounded by the radi $i R_{1}$ and $R_{1+1}$. The rings are chosen to have equal surface areas. The cross-hatched region is the portion of the leakage surface outside the outermost tally ring. 
the problem cylinder and for each ring tallies the total weight, total energy, and energy spectra of electrons and photons crossing the leakage surface within that ring. Additionally the code tallies the total weight and total energy crossing the leakage surface outside the outermost ring. This is shown as the cross-hatched region in Fig. 7. In the current version of this tally, the rings are automatically chosen to be of equal surface area. This tally is an important ingredient of the version of CYLTRAN that supports magnetic spectrometer design.

We have also included in this version of CYLTRAN a generalization of the standard source. In addition to a monodirectional source at an arbitrary angle and a source with a cosine-law distribution in the forward $(+z)$ direction, the code will now generate an isotropic source with directions contained within a cone centered on the $z$-axis with arbitrary opening angle. Figure 8 illustrates the new source option. This source is important in the correct modeling of a variety of radiographic problems with which we have been concerned.

In the memorandum $x-6: 83-7-8$, we reported these two additions to CYLTRAN, made the source files and standard updates available, and provided a test problem as a teaching aid for the interested user.

G. Monte Carlo Simulation of Electron/Photon/Cerenkov Cascades in

Generalized Geometry (J. M. Mack, X-6; and T. M. Jordan*)

Computational methods of coupled electron/photon transport are now widely used to solve a variety of radiation transport problems. 42 of these methods, Monte Carlo provides the most comprehensive treatment of cascade generation and transport in generalized geometry. Evaluating cascade production, transport and penetration through matter is the essential task. The ACCEPT 43 Monte Carlo electron/photon computer code produces cascades that include photoelectric, Compton, pair, Auger, and knock-on electrons; and bremsstrahlung, characteristic $X$-rays, and annihilation photons. Integrating Cerenkov photon production into the cascade provides a new capability to investigate certain detector concepts considered in gamina-ray and electron diagnostics.

\footnotetext{
* Present address: Experimental and Mathematical Physics Consultants, Gaithersburg, Maryland 20878.
} 


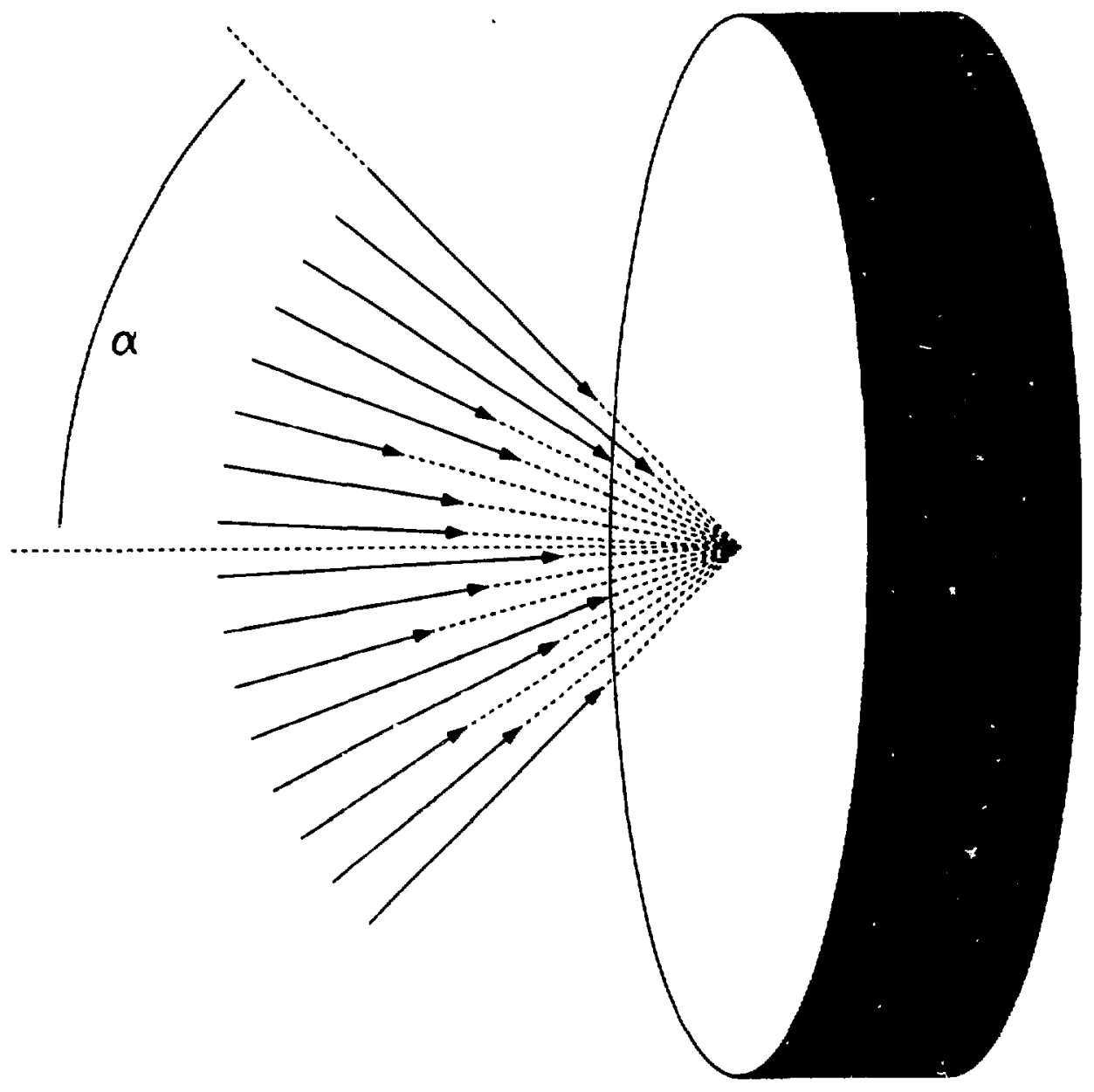

Fig. 8. The new cone source for CYLTRAN, illustrated for the case SORCIN $=0$. The value

$$
\operatorname{CTHIN}=1+\cos (\alpha)
$$

will cause this source to be generated. 
The physics of Cerenkov production was initially formulated by E'rank and Tamm. 44 The Cerenkov effect is the coherent response of a transparent medium to the passage of a charged particle whose velocity is greater than the waveiength-dependent photon phase velocity in that medium. The resulting Cerenkov electromagnetic wavefronts along the particle track constructively interfere (via Huygens' Principle) at a unique angle $\theta$ relative to the direction of the charged particle. Angle $\theta$ is defined by the Cerenkov relation 45

$$
\cos \theta=\frac{1}{\beta n(\lambda)} \text {, }
$$

Where $B$ is the ratio of particle velocity to the velocity of light in vacuum and $n(\lambda)$ is the wavelength-dependent index of refraction for that medium. For an electron, the Cerenkov number produced $\mathrm{N}$ per unit pathlength $\ell$ as $a$ function of wavelength is

$$
\frac{d N}{d l}=2 \pi \alpha \int_{\lambda_{1}}^{\lambda_{2}}\left[1-\frac{1}{\beta^{2} n^{2}(\lambda)}\right] \frac{d \lambda}{\lambda^{2}}, \lambda_{2}>\lambda_{1},
$$

where $\alpha$ is the fine-structure constant. Production increases toward shorter wavelengths and is not allowed at waveleng ths where $n(\lambda)<1 / \beta$, thereby defining the Cerenkov threshold condition.

The ACCEPT 43 computer code, chosen as the basic modeling framework, is an extension of the work of Berger. 46 It uses condensed-history Monte Carlo for electron transport and conventional single-scattering Monte Carlo for photon transport. The random walk is executed within a three-dimensional combinatorial geometry approach similar to that found in the MORSE 47 code.

Total production integrated over an assumed wavelength bandwidth and along a condensed-history pathlength is computed using Eq. (68). Emission occurs at a point randomly selected along that pathlength; the emission angle, azimuthally symmetric about the electron direction, is chosen using the Cerenkov relation, Eq. (67). The trajectory of an emitted Cerenkov photon moving through various three-dimensional zones is affected by a set of bulk 
optical properties assigned to each Monte Carlo zone. These waveleng thindependent zonal bulk properties include the refractive index, absorption coefficient, specular and diffuse reflection fractions, and fractional light transmittance. The transmittance fraction is used in conjunction with Snell's Law to determine at boundary crossings the weight fraction and direction of Cerenkov photons allowed into the next zone.

To establish model credibility, we simulated a gas Cerenkov detector (GCD) experiment reported by Brolley, et al. 48 Because the refractive index varies with gas pressure, the onset of Cerenkov production can be tuned to a specific electron threshold energy. One experinental objective was to measure, using a photodiode, the amount of Cerenkov radiation produced by an electron beam incident on a cylindrical carbon-dioxide gas cavity. Internal optics are used to collect Cerenkov photons and direct them to a photodiode mounted in a flange offset from the cylindrical gas cavity. The cylindrical cavity and internal optics were modeled in 3-D combinatorial geometry; geometry associated with the photodiode flange and mount was omitted. Not accounting for losses in the photodiode flange resulted in calculated absolute detector current overestimates.

Empirical results for 1.85 -atin carbon dioxide are shown as diamond points in Fiz. 9, where the electron beam energy ranged from 10 to $25 \mathrm{MeV}$. The circles in Fig. 9 show the results of Monte Carlo simulations using this GCD. The two sets of data are normalized to the $17-\mathrm{MeV}$ beam energy. Agreement in shape is excellent until the production threshold energy of $12 \mathrm{MeV}$ is approached, where only marginal correlation exists. In this proximity, Cerenkov production is theoretically approaching zero rapidly, making a Monte Carlo estimate with acceptable statistics prohibitive; also, experiment uncertainties are large and amorphous in the threshold region. The fast rise near the threshold energy and subsequent saturation at higher beam energy of both calculation and experiment are anticipated and consistent with the production energy dependence exhibited by Eq. (68). Although the data are shown normalized, the ratios of calculated-to-measured absolute Cerenkov detector current are within the experimental error at energy greater than $13 \mathrm{MeV}$, if the photodiode flange geometry and associated losses are included in the model. 


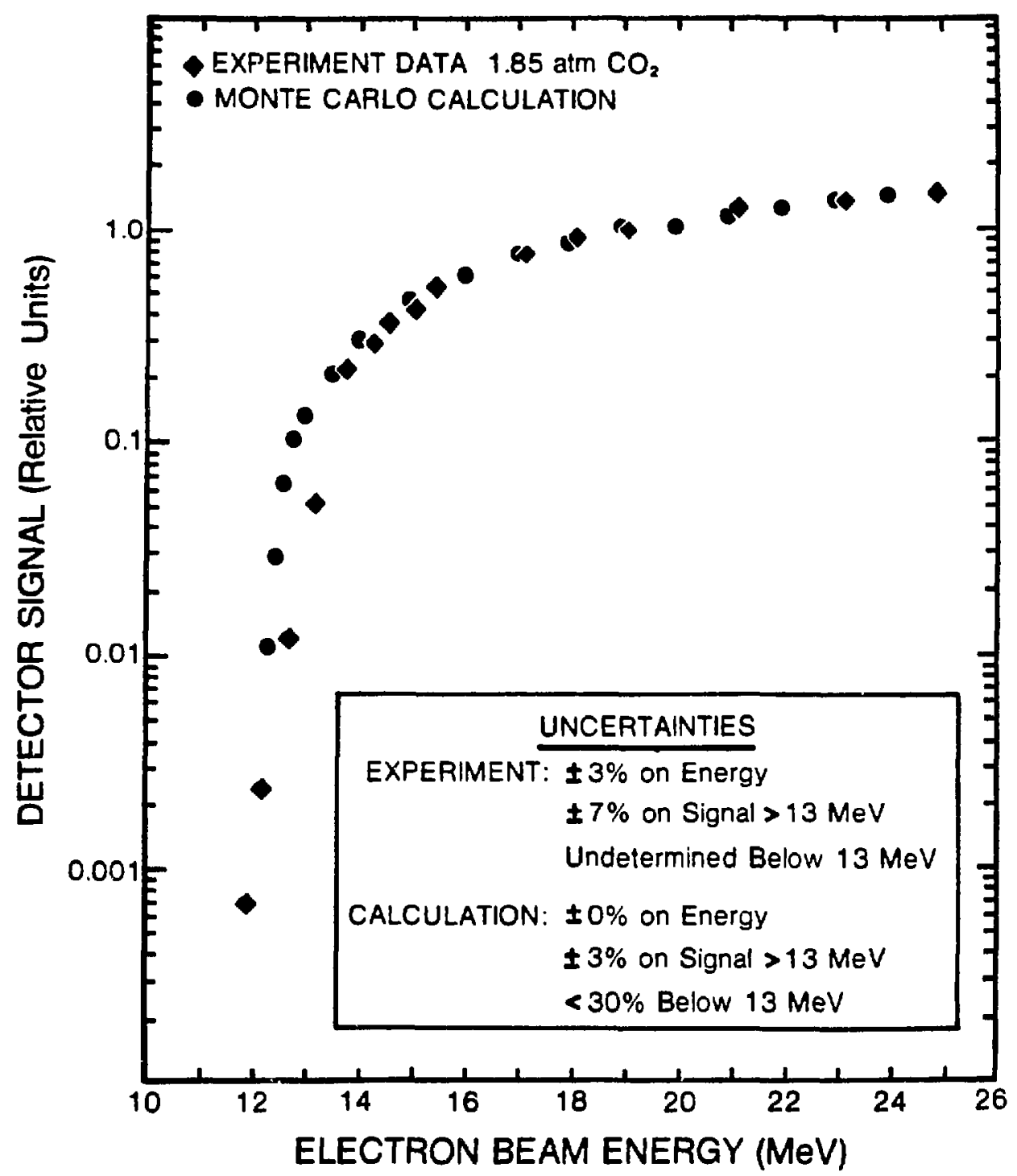

Fig. 9. Calculated and measured Cerenkov threshold for gas fill of 1.85 atm $\mathrm{CO}_{2}$. 
In summary, the coupling of Cerenkov production to the electron/photon cascade has been implemented in a generalized three-dimensional framework incorporating bulk optical properties for use in optical photon ray-tracing. The model has been used and verified successfully in design studies of gas Cerenkov detectors. Additional features being investigated for the present model are coupling to sophisticated optics design codes and accommodation of transition radiation.

H. CONPAR (J. C. Ferguson)

The CONPAR computer code 49 creates three-dimensional computer graphics displays (shaded, as well as line drawings) of geometry environments consisting of objects bounded by quadrics and torii. The algorithm is based on polygonal representations and consists of the state-of-the-art software package, MOVIE, 50 issued and maintained by Brigham Young University.

CONPAR functions as an easy-to-use interface between user and MOVIE in that geometry can be defined using simple statements. CONPAR transforms a set of user-defined constrained mathematical surfaces into the necessary polygonal geometry required by MOVIE.

Work during this reporting period consisted of extending and updating the code and its conmand language and the preparation of preliminary documentation, Ref. 49.

I. LWR Benchmark Comparative Calculations (G. P. Estes and W. T. Urban) Transport calculations have been performed for a light water reactor (LWR) shielding benchmark using the continuous energy Monte Carlo code, MCNP, 14 the multigroup Monte Carlo code, MCMG (a variant of MCNP), and the discrete-ordinates code. ONEDANT. 3 This work is part of a continuing effort at Los Alamos to benchmark and intercompare codes and data. Also this work was done in support of the ANS Committee on Shielding Benchmarks. Comparisons have been made with respect to neutron and gamma-ray dose rates, and flux spectra as calculated using the three codes. Results of this work have been reported in Ref. 51 .

The ANS Committee 6.2.1 LWR Problem No. 1 was the reactor configuration considered in this work and consisted of a one-dimensional slice through a typical LWR from the core center out to the edge of the pressure vessel. All parameters are specified except the code and cross sections to be used. Complete details regarding the power distribution were provided thereby allowing 
the problem to be run in a fixed source, coupled neutron gamma-ray mode. ENDF/B-V cross sections were used in all calculations. The continuous energy MCNP cross-section library included the new expanded neutron induced gamma-ray production treatment that provides a more accurate representation of the gamma-ray energy and angular distributions. 52 The ONEDANT and MCMG calculations used the same 30-neutron, 12 gamma-ray, infinitely dilute, multigroup cross-section set with a $P_{3}$ scattering approximation. Cross sections for ONEDANT were also transport corrected. No attempt was made to tailor the cross-section library to this particular configuration as it was our intention to determine how well an off-the-shelf cross-section library would perform in this application.

Neutron and gamma-ray dose rates calculated are compared in Table IV. In general, the MCMG and ONEDANT results are in good agreement (< 10\%) with each other for both neutron and gamma rays as would be expected. This level of good agreement is also observed between the MCNP and multigroup neutron-doserate results for the three innermost locations. However, at the pressure vessel outer radius the multigroup neutron results are $=20 \%$ lower. Although this difference is not statistically significant at the 20 level, we believe that part of the difference may be due to lack of a neutron resonance selfshielding correction in the multigroup cross sections. This conclusion is supported by spectral differences in the neutron fluxes observed in the energy range from $\approx 5.0$ to $10^{-3} \mathrm{MeV}$ where Fe resonances are significant. Furthermore, the use of a pseudo-multigroup cross-section library in MCNP yields neutron results which agree well ( $<5 \%$ ) with the multigroup code results. Finally, the flux-to-dose conversion factors are sensitive to a continuous versus multigroup treatment particularly for neutrons with energies below $1 \mathrm{MeV}$ that are the dominant contributors to the dose rate at the pressure vessel outer radius.

The MCMG and ONEDAINT gamma-ray dose rates are higher than the MCNP results by $\approx 10$ to $\approx 20 \%$. One might suspect that this is, in part, because of the new expanded ganma-ray production treatment used in MCNP. However, we do not presently have clear evidence to support such a clain. Another potential source of the observed gamma-ray differences is that the multigroup gamma-ray energy structure may be too coarse. Investigation of these differences is continuing. 
TABLE IV

I LWR SHIELDING BENCHMARK DOSE RATES' (R/HF)

N E U T RO N

C A M M A R A Y

\begin{tabular}{|c|c|c|c|c|c|c|}
\hline Location & MCNP & MCMC & ONEDANT & MCNP & MCMG & ONEDANT \\
\hline Core, OR & $5.46+9^{b}(0.023)^{c}$ & $5.61+9(0.015)$ & $5.57+9$ & $1.54+8(0.059)$ & $1.66+8(0.024)$ & $1.61+8$ \\
\hline Core barrel, IR & $4.93+8(0.040)$ & $5.12+8(0.030)$ & $4.90+8$ & $8.14+7(0.032)$ & $9.82+7(0.017)$ & $9.59+7$ \\
\hline Pressure vessel, IA & $2.61+6(0.061)$ & $2.58+6(0.084)$ & $2.34+6$ & $2.40 \cdot 6(0.042)$ & $2.90+6(0.030)$ & $2.95+6$ \\
\hline Pressure vessel, oR & $2.45+5(0.064)$ & $1.85+5(0.092)$ & $2.03+5$ & $7.20+3(0.047)$ & $7.51+3(0.105)$ & $8.32+3$ \\
\hline
\end{tabular}

aflux-to-dose conversion factor based on ANSI/ANS-6.1.1-197\%.

$5.46+9=5.46 \times 10^{9}$.

eFractional error at 10 confidence level.

Neutron and gamma-ray flux spectra have also been compared at various locations and provide a differential means of evaluating the results. Figure 10 illustrates the neutron flux comparison at the pressure vessel inner radius. Analysis of the flux spectra comparisons has provided much of the basis for the postulated reasons for the different results from the different methods.

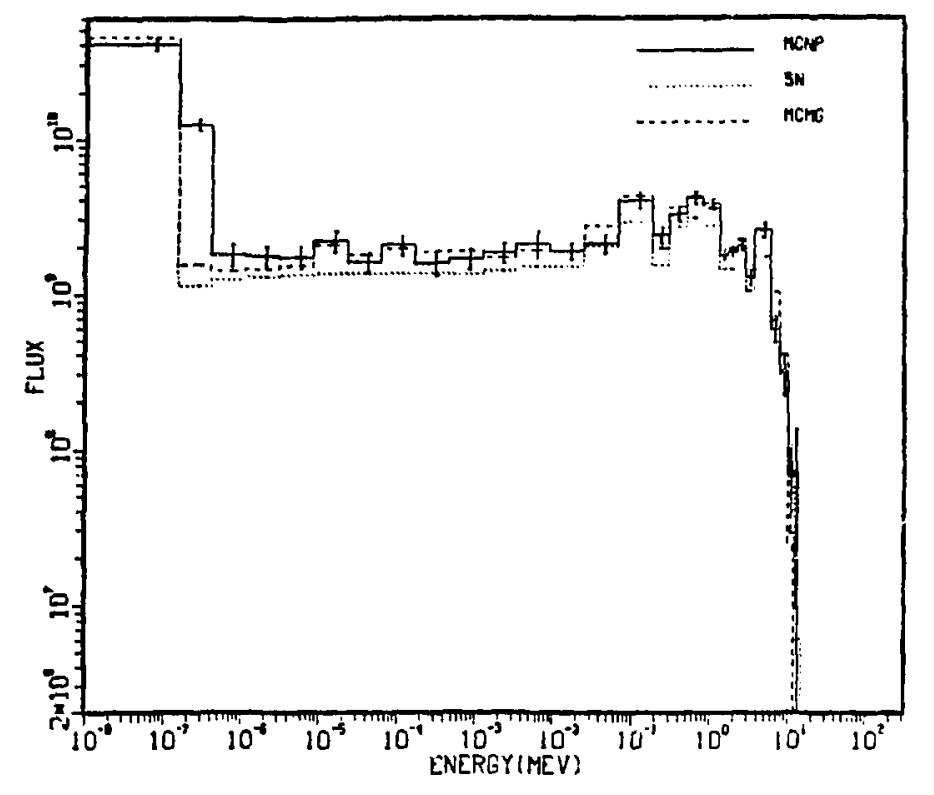

Fig. 10. Comparison of neutron flux spectra at LWR pressure vessel inner radius. 
Integral and differential results have been obtain from multigroup Monte Carlo and discrete-ordinates calculations, and a continuous energy Monte Carlo calculation for the ANS LWR shielding benchmark. The results demonstrate that reasonable neutron and gamma-ray dose rates can be calculated along the core midplane out to the pressure vessel outer radius using a 30 by 12 coupled neutron gamma-ray, off-the-shelf cross-section library.

\section{CROSS SECTIONS AND PHYSICS}

Part of our effort in Group $X-6$ is devoted to the acquisition, validation, and creation of libraries of cross sections for use in our deterministic and probabilistic codes. We also devote effort to supporting research and evaluations of phjsics models for radiation transport problems of interest.

In this section we report on biasing of neutron-induced photons in the MCNP Monte Carlo code. This is followed by a brief comment on ENDF/B-V Revision 2 cross-section data for use in our codes. Next, as part of our supporting research and evaluations of physics models, we provide a report on approxinate scattering of gamma and $x$ rays for Monte Carlo use for partially ionized targets. Also reported is an abstract of work on electron multiple, plural, and single scattering. The section concludes with a summary of calculations on the ranges of deuterons and tritons in 50:50 DT plasmas at temperatures of 1 and $10 \mathrm{keV}$.

\section{A. Biasing of Neutron-Induced Photons (R. C. Little)}

For many types of MCNP Mode 1 applications, the desired result is the intensity of a (several) discrete-energy photon(s). In these cases, it makes sense to bias the spectrum of neutron-induced photons to only produce those that are interesting.

A patch has been written to MCNP Version 3 to do this. The patch and CTSS executable are available on CFS as /090895/PIKMT/PATCH3 and /090895/ PIKMT/MCPIKMT3 for Los Alamos users.

The biasing of the induced photon spectrum is controlled by the PIKMT card which has entries as follows: 


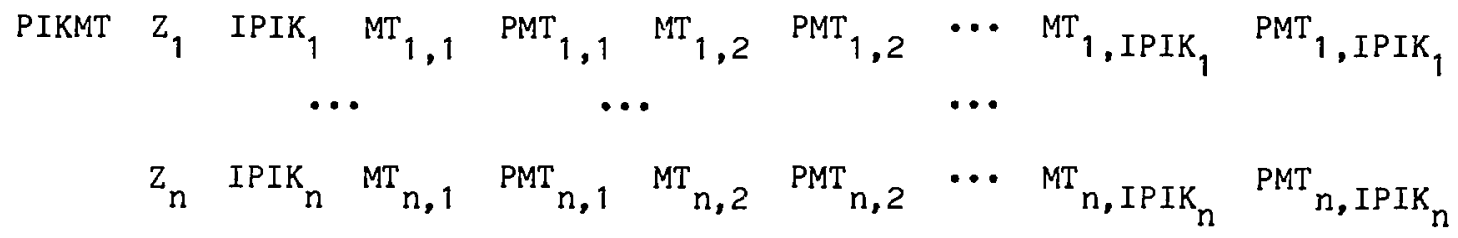

where

$z_{i}$ is the ZAID of the $i$ th entry. Full or partial ZAIDs may be specified, i.e., 29000 is equivalent to 29000.50 .

$I P I K_{i}$ is the parameter that controls the biasing for $Z A I D_{i}$. If IPIK $i=0$, there is no biasing for $Z A I D_{i}$; photons from $Z A I D_{i}$ are produced with the normal Version 3 sampling technique. If $\operatorname{IPIK}_{j}=-1$, no photons are produced from $Z A I D_{i}$. This is the default in the patch, so that if no entries are made on the PIKMT card for a particular ZAID, there will be no photons produced from that ZAID. If IPIK $j>0$, there is biasing for $Z A I D_{i}$. The value of IPIK $_{1}$ is the number of partial photon-production reactions to be sampled.

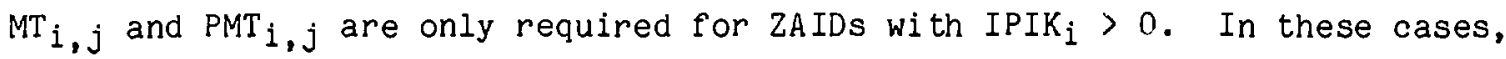
$I_{P I K_{1}}$ pairs of entries of MTs and PMTs are necessary. The MTs are the identifiers for the partial photon-production reactions to be sampled. The PMTs control, to a certain extent, the frequency with which the specified MTs are sampled. The entries need not be normalized and are described in more detail later in this memo. Any reaction that is not identified with its MT on the PIKMT card will not be sampled. We will next describe the mechanics for produclng biased neutron-induced photons.

1. Selecting Collision Isotope. In this patch, it is no longer required that the neutron collision producing a photon be with the same isotope as the neutron collision itself. Photons are only produced from isotopes called for on the PIKMT card. Two extra cross sections are calculated in AECTOT:

TOTGP2 is the total macroscopic photon-production cross section in cell IA. It includes photon production from "allowed" isotopes and "non-allowed" isotopes. 
TOTGP1 is the total macroscopic photon-production cross section in cell IA for "allowed" isotopes only. These are the isotopes on the PIKMT card with IPIK $\geq 0$.

Also in ACETOT, we calculate TOTGP(IEX), which is the microscopic photonproduction cross section for isotope IEX. TOTGP(IEX) $=0.0$ if isotope IEX is not allowed to produce photons.

In COLIDN, if TOTGP1 $=0.0$, no photon is produced. Otherwise, one photon is written to the bank for each neutron collision. The isotope producing the photon, $k$, is sampled from

$$
\sum_{i=1}^{k-1} \operatorname{TOTGP}_{i}{ }^{*} \mathrm{FME}_{i} \leq \xi^{*} \operatorname{TOTGP}<\sum_{i=1}^{k} \operatorname{TOTGP}_{i}{ }^{*} \mathrm{FME}_{i}
$$

where $F E_{i}$ is the atomic density of isotope $i$. The weight of the photon written to the bank is reduced by the factor TOTGP1/TOTM, where TOTM is the total macroscopic neutron interaction cross section.

2. Sampling Photon-Production Reaction. For these isotopes with IPIK >0, the weight of the photon is reduced by the factor GPPART/TOTGP $(k)$, where GPPART is the total cross section for those reactions allowed for isotope $k$ on the PIKMT card, and TOTGP $(k)$ is the total photon-production cross section for isotope $k$. Of course, if GPPART $=0.0$, no photon is produced. If $\operatorname{IPIK}(K)=1$, then the one reaction specified on the PIKMT card is selected. If $\operatorname{IPIK}(k)>1$, the reaction is sampled based on the cross sections for the "allowed" reactions and the PMTs on the PIKMT card. The PMTs (normalized by the code, if not by the user) do not control the absolute probabilities of choosing particular MTs, rather they alter the probabilities that would be obtained from the partial cross sections alone. If there are IPIK reactions allowed, any reaction with PMT > 1/IPIK will be sampled more frequently than its cross section alone would dictate, and any reaction with PMT > 1/IPIK would be sampled less frequently than dictated by its cross section.

The reaction sampling requires calculation of 


$$
\text { GPPAR2 }=\sum_{i=1}^{I P I K} \sigma_{i}^{* \text { PMT }_{i}} \text {, }
$$

where $\sigma_{i}$ is the cross section for photon-production reaction $i$. The reaction, $j$, is then sampled from

$$
\sum_{i=1}^{j-1} \sigma_{i}^{* P_{M T}} \leq \xi^{* \text { GPPAR2 }}<\sum_{i=1}^{j} \sigma_{i}{ }^{* \text { PMT }} .
$$

The photon's weight is then multiplied by GPPAR2/(GPPART*PMT $j)$. I don't expect the PMT option to be valuable in too many applications. To effectively ignore it, simply enter 1.0 for each PMT on the PIKMT card.

3. Notes to the user. (a) You will produce a lot of photons, many more starting photons per neutron than with the unpatched MCNP. This is because we try to write one photon to the bank at every neutron collision, rather than one photon every $\sigma_{n}, t_{0} / \sigma \gamma$, tot collisions, where $\sigma_{n}$, tot is the total neutron cross section and $\sigma \gamma$, tot is the total photon-production cross section. (The ratio $\sigma_{n}$, tot $/ \sigma \gamma$, tot assumes default PWTs.)

(b) Neutron tallies will be unaffected (within statistics), but photon tallies, of course, will be affected. The only reliable photon tallies in the biased problem are tallies with energy bins immediately around the energies of the discrete photons produced.

(c) Users need information about the MT identifiers of the reactions that produce discrete-energy photons. This information is available for Los Alamos users for isotopes on the RMCCS library in a jocument stored on CFS as 1090895/SIG/RMCCSINFO for the 7600, and /090895/SIG/RINFOC for the CRAYS. Send the file to HSP with the $-V,-U C$, and $-C C$ options.

(d) This code is also useful for biasing the neutron-induced photon spectrum to produce very high energy photons $\left(E_{\gamma}>10 \mathrm{MeV}\right)$. Without biasing, these high-energy photons are produced very infrequently; therefore, it is difficult to extract reliable statistical information about them (see R. C. Little, "The NIPE Code - A Tool to Assist in Efficient. Monte Carlo Calculations of High-Energy Neutron-Induced Photons, "Los Alamos National Laboratory internal memorandum to Distribution (Feb. 1, 1984)). 
4. Results. Several test problems have been run with the patched code at Los Alamos. Results from the simplest problem will be summarized here. The problem is an $11.2-\mathrm{cm}$ radius $C u$ sphere with a point isotropic, 0-20 MeV, neutron source in the center. Neutron and photon leakage currents are calculated. Consulting the RMCCSINFO document mentioned above I find that Cu produces (among others) discrete photons at $1.115 \mathrm{MeV}$ and $1.326 \mathrm{MeV}$. RMCCSINFO tells me that MT 3001 produces the $1.326 \mathrm{MeV}$ photon while MT 3002 produces the $1.115-\mathrm{MeV}$ photon. The photons are produced relatively infrequently and, therefore, may be difficult to sample. The results from several runs are indicated in Table $\mathrm{V}$. Note the improvement in the figure-of-merit for the photon energy bin from 1.326-1.327 MeV. As the PMT for MT 3001 is increased from 0 (no bias), to 0.1 , to 0.5 , to 0.9 , to 1.0 , the FOM increases from 75 to 103 , to 650 , to 889 , to 1111 . The dramatic increase in FOM indicates what can be done when biasing a relatively unlikely event. of course, results for a likely event are not greatly improved by biasing. Notice also that the results that are outside the domain of what we are biasing (for example, total photon leakage) are nonsense.

B. ENDF/B-V Revision 2 (R. C. Little and R. E. Seamon)

In 1981 the National Nuclear Data Center (NNDC) released Revision 1 of ENDF/B-V. We looked at all the changes in terms of their importance to the ACE-formatted Monte Carlo cross sections and replaced the data for three isotopes $(\mathrm{H}-2, \mathrm{Gd}-156, \mathrm{Au}-197)$ at that time.53 During 1983 the NNDC released Revision 2 of ENDF/B-V. In Ref. 54 we documented which of these revisions af'ect our Iibraries and asked for the reprocessing through NJOY of a total of 18 isotopes and waterials. Some of the new files have been received from Group $\mathrm{T}-2$ and $\mathrm{will}$ be checked by us before the remaining materials are processed.

C. Approximate Scattering of Camma and X Rays for Monte Carlo Use when Targets are at High Temperature (but not fully lonized) and as a Function of Density (J.J. Devaney)

The theory of photon elastic and inelastic scattering from atomic electrons at low temperature and at very high temperature (free electrons) is known. We offer a simple theory giving the photon scattering from partially 
TABLE $V$

\begin{tabular}{|c|c|c|c|c|c|c|}
\hline & \multicolumn{5}{|c|}{ RESULTS OF IEST PHORLEM } & \multirow[b]{2}{*}{$\begin{array}{l}\text { From MT } 3001 \\
\quad+ \\
\text { Photon Leakage } \\
(1.325-1.327 \mathrm{MeV})\end{array}$} \\
\hline & $\begin{array}{c}\text { Computer Time } \\
\text { (Minutes) }\end{array}$ & Source Heutrons & Total Neutron Leakage & Total Photon Leakage & $\begin{array}{l}\text { From MT } 3002 \\
+ \\
\text { Photon Leakage } \\
(1.114-1.116 \mathrm{MeV}) \\
\end{array}$ & \\
\hline No Bias & 1.54 & 22060 & $1.094 \pm 0.21 \%$ & $0.419+1.418$ & $\begin{array}{c}4.416 \mathrm{E}-3 \pm 11.24 \% \\
(\text { FOM-51) }\end{array}$ & $\begin{array}{c}7.204 \mathrm{E}-3 \pm 9.28 \% \\
(\text { FOM }=75)\end{array}$ \\
\hline $\begin{array}{l}\text { PIKMT } 290002 \\
3001 \quad .53002 \quad .5\end{array}$ & 1.54 & 15672 & $1.096 \pm 0.25 \%$ & $3.339 \mathrm{E}-2 \pm 1.47 \%$ & $\begin{array}{c}4.239 \mathrm{E}-3 \pm 3.671 \\
(\mathrm{FOM}=482)\end{array}$ & $\begin{array}{c}6.279 E-3 \pm 3.16 x \\
(\text { FOM }=6,0)\end{array}$ \\
\hline $\begin{array}{l}\text { PIKMT } 290002 \\
3001.13002 .9\end{array}$ & 1.53 & 10253 & $1.093 \pm 0.31 \%$ & $3.415 \mathrm{E}-2 \pm 2.849$ & $\begin{array}{c}4.271 \mathrm{E}-3 \pm 3.31 \% \\
(\mathrm{FOM}=597)\end{array}$ & $\begin{array}{c}6.400 E-3 \pm 7.97 \% \\
(\mathrm{FOH}=103)\end{array}$ \\
\hline $\begin{array}{l}\text { PIKMT } 290002 \\
30011 .\end{array}$ & 1.52 & 14237 & $1.985 \pm 0.26 \%$ & $3.297 E-2 \pm 2.27 x$ & $\begin{array}{c}4.753 \mathrm{E}-3 \pm 8.36 \% \\
(\mathrm{FOM}-94)\end{array}$ & $\begin{array}{c}6.069 E-3 \pm 2.729 \\
(\text { FOM }=889)\end{array}$ \\
\hline $\begin{array}{l}\text { PIKMT } 290001 \\
30021 .\end{array}$ & 2.52 & 28399 & $1.095 \pm 0.19 \%$ & $1.941 E-2 \pm 1.108$ & $2.388 \mathrm{E}-5 \pm 29.70 \%$ & $\begin{array}{c}6.041 \mathrm{E}-3 \pm 1.89 \% \\
(\text { FOM }=1111)\end{array}$ \\
\hline $\begin{array}{l}\text { PIKMT } 290001 \\
30021 .\end{array}$ & 2.52 & 25603 & $1.095 \pm 0.20 \%$ & $1.350 E-2 \pm 1.18 \%$ & $\begin{array}{c}4.296 \mathrm{E}-3 \pm 2.00 \% \\
(\mathrm{FOM}=992)\end{array}$ & 0. \\
\hline
\end{tabular}


ionized excited atoms. The number of free electrons as a function of temperature and density is obtained from the Los Alamos Equation of State Library, SESAME II. These free electrons are assumed to have a relativistic Maxwell velocity distribution. Their mean scattering can be treated by known theory for free electrons, for example by Wienke's exact theory, or by the wienkeLathrop Isotropic Approximation. The remaining (bound) electrons are treated in the standard way for atomic electrons, (see for example the Los Alamos Monte Carlo Code MCNP), except that the atomic scattering and form factors are revised to account for fewer electrons distributed ir different orbitals. The revision in these factors is obtained by first observing that the factors depend on e:ectron position solely through phase factors in which the dependency of the photon energy and the electron position variable are equivalent. Second, the first-order change in the atomic structure can be most simply and compactly represented by the mean radius of ald. the atomic electrons. Such mean radii are calculated for various elements, temperatures, and densities.

Approximate fits to the radii are made so as to span the periodic table, all temperatures, and density ratios of $1 / 5$ to 5 times normal condensed matter densities. The ratio of the mean radius to the NTP mean radius is converted to the equivalent change in the ratio of the photon energy to the incident photon energy and used to revise che atomic scattering and form factors. Using these revised factors the coherent and incoherent scattering of the remaining (bound) electrons is calculated in the normal way. The sum of this bound electrons scactering plus the free electrons scattering gives the total atomic scattering as a function of temperature and density. The simple sum is appropriate because the free electron scattering is incoherent.

A detailed report on this work is in preparation. 55

D. Electron Multiple, Plural, and Single Scattering (J. J. Devaney)

The angular distribution of a bean of electrons penetrating an infinite slab is studied with a view to providing simple accurate formulae for use in electron transport codes. Success is reported, but a highly disappointing success, for althoigh the literature is vast, (over 150 papers), the experiments are limited, sparse, and often poorly described. The theory suffers from complexity, from incomplete, erroneous, or even absent comparisons with other theories and with experiment. Consequently, one cannot say with any 
assurance what the best theory is, nor to any great accuracy, especially in limiting cases, how good the theory we recommend is. Comparisons are given with the known experiments.

With that caveat, the theory of Moliere as given by Bethe and by Hanson et al. is recommended. Formulas are given. Accuracies of a few percent for multiple scattering are expected. For the (in the cases studies) less dominant concomitant plural and single scattering the theory cannot do better than 10 to $30 \%$. Because of the complexity of the functions, either series or tables must be employed.

A bright spot in this somewhat gloomy state of affairs is the calculation of the $1 / e$ width which can be done accurately $(s 2 \%)$ by a simple approximation due to Hanson et al.

Using theories of Hanson et al. and variants thereof, an approximate "Gaussian" gives a good $(7-20 \%)$ simple description of the multiple scattering. Approximate single scattering formulis are also offered.

The details of this study are provided in a report currently in preparation. 56

E. Ranges of Deuterons and Tritons in 50:50 DT Plasmas at Temperatures of 1 and $10 \mathrm{keV}$. Energy Loss per Unit Distance (J. Devaney)

We calculate the charged particle energy losses and ranges in a 50:50 DT plasma at temperatures of $1 \mathrm{keV}$ and $10 \mathrm{keV}$ for $10 \mathrm{MeV}, 3 \mathrm{MeV}$, and $1 \mathrm{MeV}$ deuterons and tritons incident. The processes included are electronic, nuclear coulomb, hadronic, and their interference. The results are good only when collective effects are negligible, that is when intensities are not so large that electric and magnetic fields are significant or plasma oscillations are important. These latter cases usually require individual analyses.

We define and estimate, according to G. Cooper, 57 a net range end point energy at which the incident particle is expected to suffer a mean $90^{\circ}$ deflection. That end point energy, E, corrected for the effects of nuclear forces and nuclear Coulomb scattering is

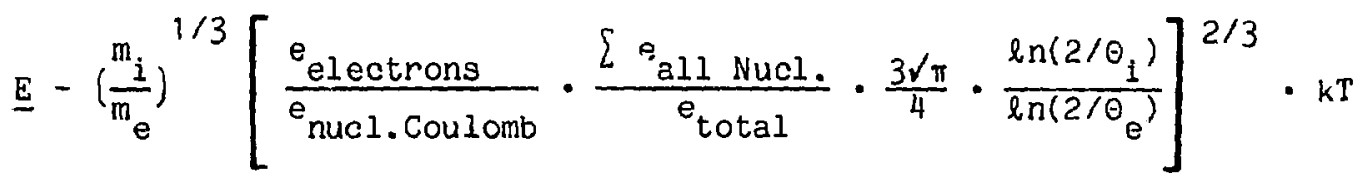


where $e \equiv d E / d x$ is the energy loss. The subscript "all Nucl.", for example, sums the energy losses from hadronic, nuclear Coulomb and their interference. in is mass, $\ell n(2 / \theta)$ is the Coulomb logarithm of energy loss theory, i refers to ions (here deuterons or tritons), and e to electrons.

The energy loss terms were calculated from the theory of C. L. Longmire,58 J. J. Devaney, and M. L. Stein, 59 and J. J. Devaney. 60

The energy loss values are given in Table VI and Figs. 11 and 12. The net range end point energies calculated are given in Table VII. We integrate the reciprocals of the total energy loss to the appropriate end point energies in order to obtain the mean particle path-lengths in our DT plasmas. The results for a plasma density of $6 \mathrm{~g} / \mathrm{cm}^{3}$ are given in Table VIII. We also include other end point energies. Errors are estimated to be in the neighborhood of $10 \%$ for path-lengths from $10 \mathrm{MeV}$ to about $0.35 \mathrm{MeV}$ and perhaps about $35 \%$ below $1 \mathrm{MeV}$.

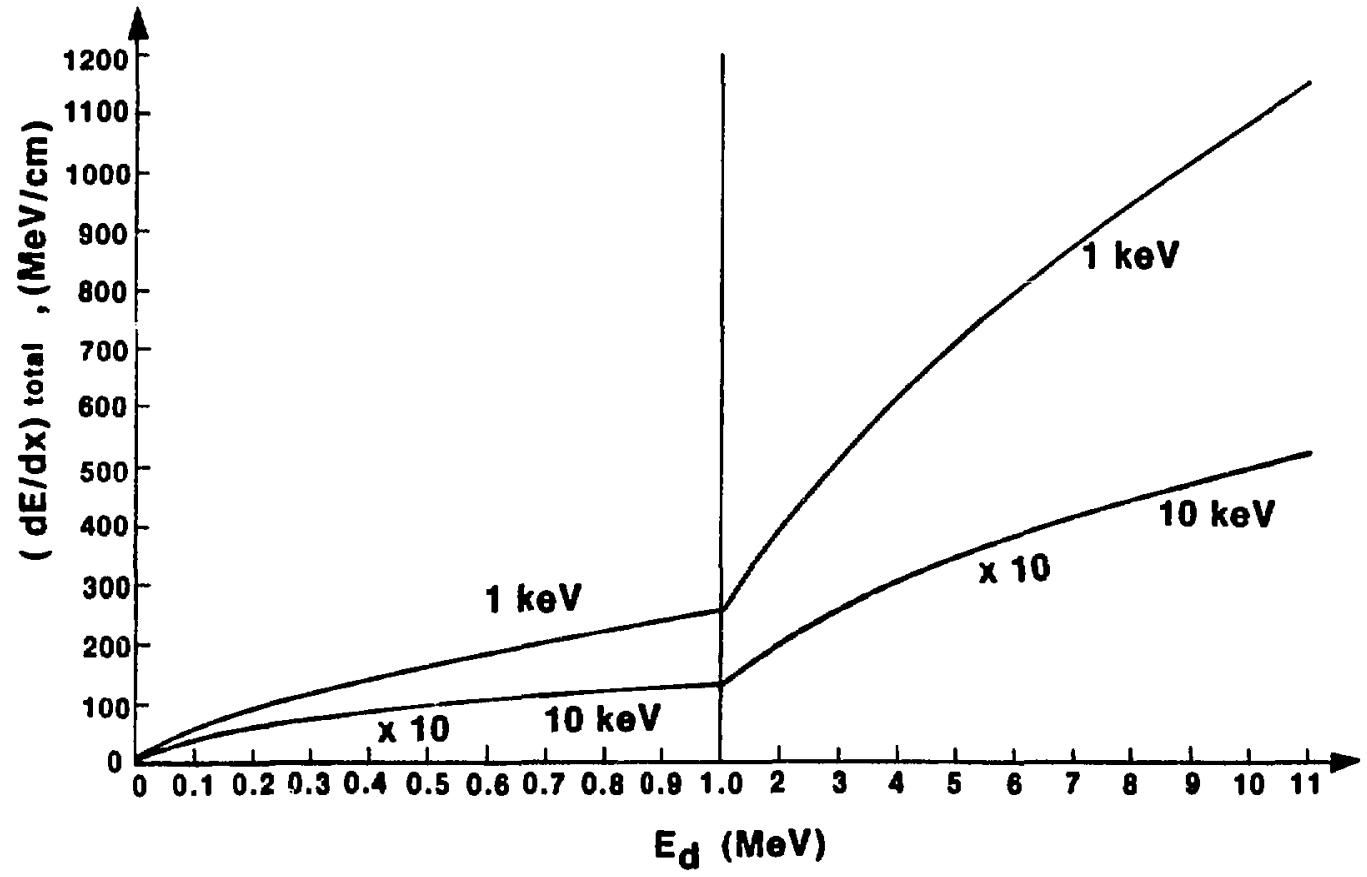

Fig. 11. Total energy loss with distance for deuterons incident on 50:50 DT at 1 and $10 \mathrm{keV}$ temperatures, density of $6 \mathrm{~g} / \mathrm{cm}^{3}$. 


\section{TABUE VI}

ENERGY LOSSES VERSUS SELECTED INCIDENT ENERGIES

$$
\text { (50:50 DT at } p=6 \mathrm{~g} / \mathrm{cm}^{3} \text { ) }
$$

Energy Loss $\frac{d E}{d x}(M e V / c m)$. Selected Incident Energy, E(MeV)

\begin{tabular}{|c|c|c|c|c|c|c|c|c|c|c|}
\hline & 10 & 5 & 2 & 1 & 0.5 & 0.2 & 0.1 & 0.05 & 0.02 & 0.01 \\
\hline \multicolumn{11}{|l|}{ Deuterons Incident DT, $\mathrm{kT}=1 \mathrm{keV}$} \\
\hline $\begin{array}{l}\text { ro electrons } \\
\text { (all quantum mechanical) }\end{array}$ & 1080.9 & 707.2 & 399.5 & 256.9 & 163.4 & 87.94 & 53.78 & 31.93 & 14.85 & 7.49 \\
\hline $\begin{array}{l}\text { to deuterium ions (Coulomb) } \\
\text { (part quantum part classical) }\end{array}$ & 1.1 & 1.1 & 1.1 & 1.0 & 1.0 & 0.96 & 0.89 & 0.83 & 0.75 & 0.69 \\
\hline to tritons (Coulomb) & 0.8 & 0.8 & 0.7 & 0.7 & 0.7 & 0.65 & 0.61 & 0.56 & 0.51 & 0.47 \\
\hline $\begin{array}{l}\text { to deuterons } \\
\text { (nuclear and interference) }\end{array}$ & 1.6 & 1.2 & 0.6 & 0.5 & 0.2 & 0.06 & 0.03 & - & - & - \\
\hline $\begin{array}{l}\text { Lo tritons } \\
\text { (nuclear and interference) }\end{array}$ & 3.5 & 3.4 & 1.1 & 0.8 & 0.6 & 0.34 & 0.01 & - & - & - \\
\hline TOTAL & 1087.9 & 713.6 & 403.0 & 259.9 & 165.9 & 89.95 & 55.32 & 33.32 & 16.11 & 8.65 \\
\hline \multicolumn{11}{|c|}{ Eeuterons Incident DT, $k T=10 \mathrm{keV}$} \\
\hline to electrons & 42.58 & 28.27 & 16.30 & 10.65 & 6.86 & 3.70 & 2.19 & 1.147 & 0.222 & - \\
\hline to deuterons (Coulomb) & 1.25 & 1.21 & 1.17 & 1.14 & 1.11 & 1.06 & 1.01 & 0.928 & 0.900 & - \\
\hline to tritons (Coulomb) & 0.85 & 0.82 & 0.79 & $0.7 i$ & 0.75 & 0.72 & 0.68 & 0.646 & 0.603 & - \\
\hline $\begin{array}{l}\text { to deuterons } \\
\text { (nuclear and interference) }\end{array}$ & 1.55 & 1.15 & 0.62 & 0.51 & 0.20 & 0.06 & 0.03 & - & $\cdot$ & - \\
\hline $\begin{array}{l}\text { to tritong } \\
\text { (nuclear and incerference) }\end{array}$ & 3.54 & $3.4 \mathrm{~g}$ & 1.09 & 0.80 & 0.00 & 0.24 & 0.01 & - & - & - \\
\hline TOTAL & 49.70 & 34.84 & $1 y .97$ & 19.81 & $y .58$ & 5.98 & 3.42 & 2.75 & 1.725 & - \\
\hline
\end{tabular}




\section{TABLE VI (Cont)}

ENERGY LOSSES VERSUS SELECTED INCIDENT ENERGIES

$$
\text { (50:50 Dr at } p=6\left(\mathrm{~B} / \mathrm{Cs}^{3}\right)
$$

Energy Loss $\frac{d E}{d x}$ (MeV/ca). Selected Incident Energy, EiMeV)

\begin{tabular}{|c|c|c|c|c|c|c|c|c|c|c|}
\hline & 10 & 5 & 2 & 1 & 0.5 & 0.2 & 0.1 & 0.05 & 0.02 & 0.0 \\
\hline \multicolumn{11}{|c|}{ Trittons Incigent UT, $k T=1$ keV } \\
\hline to electrons & 845.0 & 550.9 & 309.4 & 197.8 & 125.0 & 66.49 & 40.18 & 23.46 & 10.55 & 5.09 \\
\hline to deuterons (coulomb) & 1.7 & 1.7 & 1.6 & 1.5 & 1.5 & 1.40 & 1.31 & 1.21 & 1.09 & 1.01 \\
\hline to tritons (Coulomb) & 1.2 & 1.1 & 1.1 & 1.1 & 1.0 & 0.96 & 0.89 & 0.83 & 0.75 & 0.69 \\
\hline $\begin{array}{l}\text { to deut arons } \\
\text { (nuclear and interference) }\end{array}$ & 5.6 & 2.9 & 1.3 & 1.0 & 0.7 & 0.19 & 0.03 & - & - & - \\
\hline $\begin{array}{l}\text { to tritons } \\
\text { (nuclear and incerference) }\end{array}$ & 0.4 & 0.3 & 0.1 & 0.1 & 0.1 & 0.03 & 0.01 & - & - & - \\
\hline TOTAL & 853.8 & 556.8 & 313.5 & 201.5 & 128.3 & 69.07 & 42.41 & 25.50 & 12.39 & 6.79 \\
\hline \multicolumn{11}{|c|}{ Tritons Incident DT, $k T=10 \mathrm{kEV}$} \\
\hline to electrons & 33.59 & 22.25 & 16.79 & 8.32 & 5.34 & 2.86 & 1.684 & 0.878 & 0.17 & - \\
\hline to deuterons (Coulomb) & 1.86 & 1.82 & 1.75 & 1.70 & 1.65 & 1.58 & 1.493 & 1.419 & 1.34 & - \\
\hline to tritons (Coulomb) & 1.27 & 1.23 & 1.19 & 1.16 & 1.13 & 1.07 & 1.012 & 0.958 & 0.90 & - \\
\hline $\begin{array}{l}\text { to deuterons } \\
\text { (nuclear and interference) }\end{array}$ & 5.59 & 2.88 & 1.31 & 1.02 & 0.13 & 0.19 & 0.026 & - & - & - \\
\hline $\begin{array}{l}\text { to tritons } \\
\text { (nuclear and interference) }\end{array}$ & 0.41 & 0.28 & 0.15 & 0.12 & 0.08 & 0.03 & 0.007 & 0.001 & - & - \\
\hline TOTAL & 42.72 & 28.46 & 17.18 & 12.33 & 8.93 & 5.73 & 4.222 & 3.256 & 2.41 & - \\
\hline
\end{tabular}




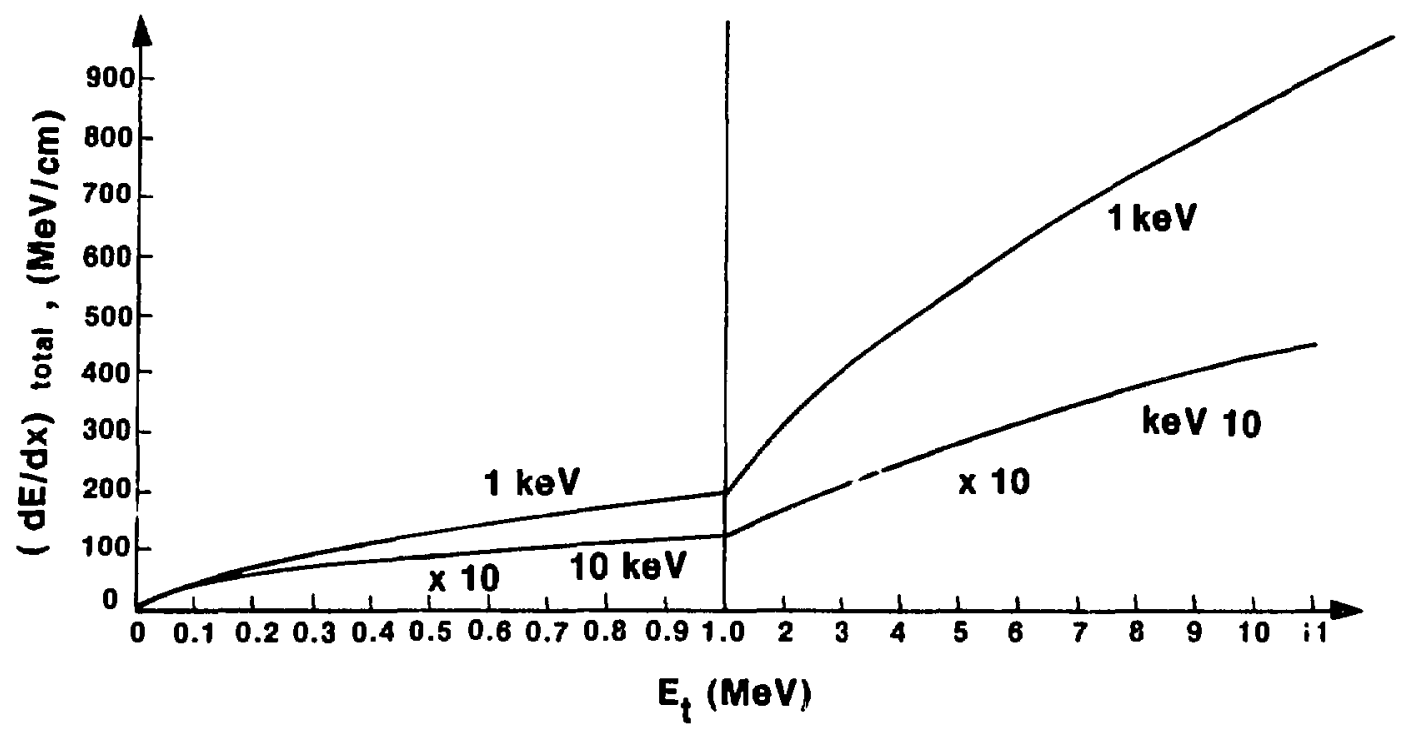

Fig. 12. Total energy loss with distance for tritons incident on 50:50 DT at 1 and $10 \mathrm{keV}$ temperatures, density of $6 \mathrm{~g} / \mathrm{cm}^{3}$.

\section{TABLE VII}

LOWER LINEAR LIMIT ENERGIES, E

(Mean Forward Range Limit)

(keV)

50:50 DT Plasma, density $\rho=6 \mathrm{~g} / \mathrm{cm}^{3}$

\begin{tabular}{lll} 
Plasma temperature: & $1 \mathrm{keV}$ & $10 \mathrm{keV}$ \\
\hline Deuterons Incident: & $50 \mathrm{keV}$ & 360 \\
Tritons Incident: & 60 & 340
\end{tabular}


TABLE VIII

PATH-LENGTHS ( $>$ RAMGES) IN CENTIMETERS OF DEUTERONS ARD THITONS

IN $6 \mathrm{G} / \mathrm{CM}^{3}$ OF $50: 50$ DT $\mathrm{MT}$ TEMPEAMTURES OF 1 AND $10 \mathrm{kEV}$

Final Particle Energy, E (Mev)

\begin{tabular}{|c|c|c|c|c|c|c|c|}
\hline $\begin{array}{l}\text { Initial } \\
\text { Particle. } \\
\text { Energy } \\
\text { E(MeV) }\end{array}$ & $\begin{array}{l}0.30 \\
\text { (Deuteron He.tn } \\
\text { Forward Range, } \\
\mathrm{KT}=10 \mathrm{keV} \text { ) }\end{array}$ & $\begin{array}{l}0.34 \\
\text { (Tritan Mean } \\
\text { Forward Kange, } \\
\mathrm{KT}=10 \mathrm{keV})\end{array}$ & 0.1 & $\begin{array}{l}0.06 \\
\text { (Tritun Mean } \\
\text { (Forwatod Fangt. } \\
k T=1 \text { keV) }\end{array}$ & $\begin{array}{l}0.05 \\
\text { (Deuteron Mean } \\
\text { forward fange, } \\
\mathrm{KT}=1 \mathrm{keV} \text { ) }\end{array}$ & 0.03 & 0.02 \\
\hline \multicolumn{8}{|c|}{ DT Temperiture $=1 \mathrm{kcV}$} \\
\hline Deuteron 10 & & & 0.0202 & & 0.0213 & & \\
\hline Triton 10 & & & $0 . J 26$ & 0.0271 & & & \\
\hline Deuteron 3 & & & 0.0114 & & 0.0125 & & \\
\hline Triton 3 & & & 0.0147 & 0.0158 & & & \\
\hline Deuteron 1 & & & 0.0061 & & 0.0073 & & \\
\hline Triton 1 & & & 0.0080 & 0.0091 & & & \\
\hline \multicolumn{8}{|c|}{ DT Temperature $=10 \mathrm{keV}$} \\
\hline Deuteron 10 & 0.347 & & 0.391 & & & & 0.419 \\
\hline Triton 10 & & 0.406 & 0.448 & & & 0.529 & \\
\hline Deuteron 3 & 0.163 & & 0.207 & & & & 0.235 \\
\hline Iriton 3 & & 0.187 & 0.228 & & & 0.309 & \\
\hline Deuteron 1 & 0.059 & & 0.103 & & & & 0.131 \\
\hline Triton 1 & & 0.066 & 0.108 & & & 0.188 & \\
\hline
\end{tabular}

Hote: The third figure is for comparison only and is not significant.

$\begin{array}{ll}0.0233 & 0.0242 \\ 0.0303 & 0.0313 \\ 0.0145 & 0.0156 \\ 0.019 & 0.0201 \\ 0.0093 & 0.0102 \\ 0.0123 & 0.0133\end{array}$




\section{REFERENCES}

1. W. F. Walters, R. D. O'Dell, and F. W. Brinkley, Jr., "THREETRAN(hex,z) User's Manual," Los Alamos Scientific Laboratory report LA-8089-M (October 1979).

2. D. R. Ferguson and K. L. Derstine, "Optimized Iteration Strategies and Data-Management Considerations for Fast Reactor Finite Difference Diffusion Theory Codes," Nucl. Sci. Eng. 64, No. 2, 593-604 (1977).

3. R. D. O'Dell, F. W. Brinkley, Jr., and D. R. Marr, "User's Manual for ONEDANT: A Code Package for One-Dimensional, Diffusion-Accelerated Neutral-Particle Transport," Los Alamos National Laboratory report LA-9184-M (February 1982).

4. R. E. Alcouffe, F. W. Brinkley, Jr., D. R. Marr, and R. D. O'Dell, "User's Guide for TWODANT: Two-Dimensional Diffusion Accelerated Neutral Particle Discrete-Ordinates Transport Code," Los Alamos National Laboratory report LA-10049-M (in press).

5. R. D. O'Dell, "Standard Interface Files and Procedures for Reactor Physics Codes, Version IV," Los Alamos Scientific Laboratory report LA-6941-MS (Sep tember 1977).

6. B. J. Toppel, "A User's Guide for the REBUS-3 Fuel Cycle Analysis Capability," Argonne National Laboratory report ANL-83-2 (March 1983).

7. W. H. Reed, "TIMEX: A Time-Dependent Explicit Discrete Ordinates Program for the Solution of Multigroup Transport Equations," Los Alamos Scientific Laboratory report LA-4800 (July 1972).

8. T. R. Hill and W. H. Reed, "TIMEX: A Time-Dependent Explicit Discrete Ordinates Program for the Solution of Multigroup Transport Equations with Delayed Neutrons," Los Alamos Scientific Laboratory report LA-6201-MS (February 1976).

9. W. H. Reed, "A Non-Iterative Method for Time Dependent Transport," in Proceedings of Conference on New Developments in Reactor Mathematics and Applications, Idaho Falls, Idaho; March 29-31, 1971, CONF-710302, pg. 679-700.

10. S. A. Dupree, H. A. Sandmeier, G. E. Hansen, W. W. Engle, Jr., and F. R. Mynatt, "Time Dependent Neutron and Photon Transport Calculations Using the Method of Discrete Ordinates, "Los Alamos Scientific Laboratory report LA-4557 (August 1970).

11. B. G. Carlson and K. D. Lathrop, "Transport Theory - Models of Discrete Ordinates," Chapter III of Computing Methods in Reactor Physics (Gordon and Breach, NY, 1968), p. 173 .

12. T. R. Hill, "ONETRAN: A Discrete-Ordinates Finite Elemerc Code for the Solution of the One-Dimensional Multigrolip Transport Equation, "Los Alamos Scientific Laboratory report LA-5990-MS (June 1975). 
13. T. R. Hill, "Efficient Methods for Time Absorption ( $\alpha$ ) Eigenvalue Calculations," Los Alamos National saboratory report LA-9602-MS (March 1983).

14. Los Alamos Monte Carlo Group, "MCNP - A General Monte Carlo Code for Neutron and Photon Transport, Version 2B," Los Alamos National Laboratory report LA-7396-M (revised) (April 1981).

15. G. C. Pomraning, The Equations of Radiation Hydrodynamics (Pergamon Press, Oxford, 1973).

16. J. S. Chang and G. Cooper, "A Practical Difference Scheme for Fokker-Planck Equations," J. Comp. Phys. 6, 1 (1970).

17. E. W. Larsen, C. D. Levermore, G. C. Pomraning, and J. G. Sanderson, "Discretization Methods for Fokker-Planck Operators," in preparation.

18. Raymond E. Alcouffe, Bradley A. Clark, and Edward W. Larsen, "The Diffusion Synthetic Acceleration of Transport Iterations with Application to a Radiation Hydrodynamics Problem," in Computational Techniques: Multiple Time Scales, J. U. Brackbill and B. I. Cohen, Eds. (to be published).

19. Charles W. Cranfill, "EOSPAC: A Subroutine Package for Accessing the Los Alamos Sesame EOS Data Library," Los Alamos National Laboratory report LA-9728-M (August 1983).

20. W. A. Lokke and W. H. Grasberger, "XSNQ-U -- A Non LTE Emission and Absorption Coefficient Subroutine," Lawrence Livermore Laboratory report UCRL-52276 (June 1977).

21. R. E. Alcouffe, "Diffusion Synthetic Acceleration Methods for the Diamond-Differenced Discrete-Ordinates Equations," Nucl. Sci. Eng. 64, 344 (1977).

22. W. F. Miller, Jr., "Generalized Rebalance: A Common Framework for Transport Acceleration Methods," Nucl. Sci. Eng. 65, 226 (1978).

23. E. W. Larsen, "Unconditionally Stable Diffusion-Synthetic Acceleration Methods for the Slab Geometry Discrete-Ordinates Equations. Part I: Theory," Nucl. Sci. Eng. 82, 47 (1982).

24. E. W. Larsen, "Diffusion-Synthetic Acceleration Methods for the Discrete-Ordinates Equations," Proc. ANS Topical Meeting, Advances in Reactor Computations, March 28-30, 1983, Salt Lake City, 2, 705 (1983).

25. E. M. Gelbard and L. A. Hageman, "The Synthetic Method as Applied to the $S_{\mathrm{N}}$ Equations," Nucl. Sci. Eng. 37, 288 (1969).

26. D. J. Dudziak, R. D. O'Dell, and R. E. Alcouffe, "Transport and Reactor Theory, October 1-December 31, 1981," Los Alamos National Laboratory report LA-9336-PR (May 1982). 
27. T. J. Seed, "TRIDENT-CTR User's Manual," Los Alamos Scientific Labnnatory report LA-7835-M, revised (November 1979).

28. Bradley A. Clark, W. T. Urban, and Donald J. Dudziak, "Evaluation of the Streaming Matrix Hybrid Method for Discrete-Ordinates Duct Streaming Calculations," Proc. Sixth Int. Conf. Radiation Shielding, Tokyo, Japan, 1983 (Japan Atomic Energy Research Institute, Tokai-mura, Japan) Vol. II, p. $852(1983)$.

29. T. N. K. Godfrey, "Repeated Structures in MCNP," Los Alamos National Laboratory (Group X-6) memorandum, November 16, 1983.

30. T. N. K. Godfrey, "Column Input for MCNP," Los Alamos National Laboratory (Group X-6) memorandum, May 23, 1983.

31. W. L. Thompson, "Qualification of the MCNP Code," Los Alamos National Laboratory (Group X-6) memorandum to D. R. Koenig (May 13, 1983).

32. R. C. Little and R. E. Seamon, "New Version of MCPLIB1D," Los Alamos National Laboratory (Group $X-6$ ) memorandum to T. N. K. Godfrey (August 5 , 1983).

33. R. E. Seamon, "Version 2C Testing," Los Alamos National Laboratory (Group $\mathrm{X}-6$ ) memorandum to $\mathrm{W}$. L. Thompson (March 30, 1982).

34. Guy P. Estes and R. G. Schrandt, "A Generalized Source Sampling Concept for MCNP and Applications in Radiation Transport," Trans. Am. Nuc. Soc. 45, 628 (1983).

35. T. E. Booth, "Alitomatic Importance Estimation in Forward Monte Carlo Calculations," Trans. Am. Nucl. Soc. 4i, 308 (1982).

36. T. E. Booth and J.S. Hendricks, 'Importance Estimation in Forward Monte Carlo Calculations," Nuclear Technology/Fusion 5, No. 1, 90 (January 1984).

37. J. S. Hendricks, "A Code-generated Monte Carlo Importanee Function," Trans. Am. Nucl. Soc. 41, 307 (1982).

38. T. E. Booth, "A Weight Window/Importance Generator for Monte Carlo Streaming Problems," Proc. Sixth Int. Conf. Radiation Shieldirig, Tokyo, May $16-20,1983$.

39. S. R. Dwivedi, "A New Importance Biasirz Scheme for Deep-Penetration Monte Carlo," Ann. Nuclear Energy 90, 359-368 (1982).

40. L. L. Carter, J. S. Hendricks, "Biasing Anisotropic Scattering Kernels for Deep-Penetration Monte Carlo Calculations," Trans. Am. Nucl. Soc. 45, 603 (November 1983).

41. J. A. Halbleib, Sr. and W. H. Vandevender, "CYLTRAN: A Cylindrical Geometry Nultimaterial Electron/Photon Monte Carlo Transport Code," Sandia Laboratories report SAivD-74-0030 (1974). 
42. Joseph M. Mack, "Computational Methods of Electron/Photon Transport," American Nuclear Society Topical Meeting, Advances in Reactor Computations, ISBN: 0-89448-111-8, p. 595, Salt Lake City, Utah (March 1983).

43. John Halbleib, Sr., "ACCEPT: A Three-Dimensional Electron/Photon Monte Carlo Transport Code Using Combinatorial Geometry," Sandia National Laboratories, Albuquerque, New Mexico, SAND-79-0415 (May 1979).

44. I. M. Frank and Ig. Tamm, Dokl. Akad. Nauk., SSSR 14 (3), 109 (1937).

45. J. V. Kellye, Cerenkor Radiation (Pergamon Press, New York, 1958).

46. M. J. Berger, "Monte Carlo Calculation of the Penetration and Diffusion of Fast Charged Particles," in Methods in Computational Physics, Vol. 1 (Academic Press, New York, 1963).

47. E. A. Straker, W. H. Scott, Jr., and N. R. Byrn, "The MORSE Code with Combinatorial Geometry," Science Applications, Inc., SAI-72-511-LJ (DNA 2860T) (May 1972).

48. John E. Brolley, Peter B. Lyons, Joseph M. Mack, James W. Toevs, Carl S. Young, S. Iversen, P. E. Nash, P. A. Zagarino, and R. D. Seno, "A Gas Cerenkov Detector for Measuring $16.7 \mathrm{MeV} \gamma$-Rays from the $D(t, \gamma) \mathrm{He}$ Reaction," Los Alamos National Laboratory, Conference Optics '83, Santa Fe, New Mexico, April 11-15, 1983.

49. J. C. Ferguson, "CONPAR, A Constrained Parametric Surface 3-D Graphics Program," Los Alamos National Laboratory (Group X-6) memorandum (January 1984).

50. "MOVIE: A General Purpose Computer Graphics System," Computer Graphics document GR834, Los Alamos National Laboratory (August 1982).

51. W. T. Urban ani Juy P. Estes, "LWR Shielding Benchmark Cajculations," Trans. Am. Nucl. Soc. 45, 588 (1983).

52. Robert C. Little and Rober E. Seamon, "Neutron-Induced Photon Production in MCNP," Trans. Am. Nucl. Soc. 43, 627 (1982).

53. R. C. Little and R. E. Seamon, "Current Status of the Monte Carlo CrossSection Files with Emphasis on the Sources of the Basic ENDF/B-V Data," Los Alamos National Laboratory (Group $X-6$ ) memorandum to $P$. G. Young and E. D. Arthur, Chairmen, CSEWG Evaluations Committee (June 12, 1981).

54. R. C. Little and R. E. Seamon, "ENDF/B-V Revision 2 Cross Sections for MCNP," LoS Alamos National Laboratory (Group X-6) memorandum (November. $30,1983)$. 
END

DATE FILNED 02/19/55 